AUTHOR

TITLE

INSTITUTION

SPONS AGENCY

PUB DATE

NOTE

CONTRACT

AVAILABLE FROM

PUB TYPE

EDRS PRICE

DESCRIPTORS

IDENTIFIERS
Parker, Faith Lamb; Piotrkowski, Chaya S.; Kessler-Sklar, Susan; Baker, Amy J. L.; Peay, Lenore; Clark, Beryl The Impact of Parent Involvement in Head start on Parents and Children. Final Report [and] Executive Summary. National Council of Jewish Women, New York, NY. Center for the Child.; Yale Univ., New Haven, CT. Bush Center in Child Development and Social Policy.

Administration for Children, Youth, and Families (DHHS), washington, DC. Project Head Start.; Ford Foundation, New York, NY.

1997-00-00

213p.; Prepared in collaboration with the Ft. George Community Enrichment Center, Inc., and staten Island Mental Health Services Head start.

90-CD-0733/01-3

National Council of Jewish women Center for the Child, 53 West 23rd Street, New York, NY 10010; Tel: 212-645-4048; Fax: 212-645-7466.

Reports - Descriptive (141)

MFO1/PCO9 Plus Postage.

Child Development; Early Childhood Education; Individual Characteristics; Longitudinal studies; Parent Influence;

* Parent Participation; Parents; Preschool Education;

* Program Effectiveness; Program Evaluation

* Project Head start

ABSTRACT

From its inception, Head Start's legislative mandate called for "maximum feasible participation" of parents in all programmatic efforts and policy decisions. Nevertheless, there has been little research done on the benefits of Head start to parents and on the role of parents as mediators of child and family outcomes. The Head start Parent Involvement Project in 1990 explored this virtually untapped area. This five-year initiative consisted of three parts: (1) a parent survey exploring life events and experiences that might function as barriers to parents' participation in Head start; (2) a longitudinal study of parent involvement in Head start assessing its impact on parents, Head start children, and their siblings; and (3) an intervention focused on staff development and training to enhance staff's work with Head start parents. The executive summary and final report describe each part of the initiative and its results. Major findings on parental participation in Head start include: (1) that it improves family life through improved parent-child relationships, enhanced home learning environments, children's greater social competence, greater parental involvement in elementary school, and increased parental self-sufficiency; and (2) that parents who get involved in Head start reported needing education, job training, or paid employment at the beginning of the Head start year; were not pregnant; were not engaged in self-sufficiency promoting activities; had greater expectations for their child's long-term school success; and had a younger child in Head start. The summary includes policy and program recommendations. Eighteen tables and three figures are appended to the final report. The final report contains 78 references. (EV) 


\title{
- FINAL REPORT
}

\section{THE IMPACT OF PARENT INVOLVEMENT IN HEAD START ON PARENTS AND CHILDREN}

\author{
Authors: \\ Faith Lamb Parker \\ Chaya S. Piotrkowski \\ Susan Kessler-Sklar \\ Amy J. L. Baker \\ Lenore Peay \\ Beryl Clark \\ Prepared by: \\ NCJW Center for the Child \\ In Collaboration With:
}

The Bush Center in Child Development and Social Policy, Yale University

Ft. George Community Enrichment Center, Inc.

Staten Island Mental Health Services Head Start

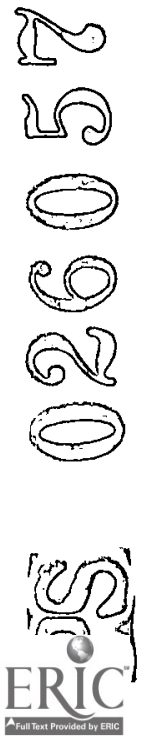

This study was funded $\mathrm{n}$ part by the United States Department of Iealth $\&$ Human Serviees, Administration on Children, Youth and Families (Grant No, 90000733013$)$, the Ford Foundation, the National council of lewish Women ond another private foundation. (Rederal funding for this study totaled $\$ 300000$ ropresenting approximatel $53 \%$ of the profect's total budget) THE OPINTONS EXPRESSED IN THIS DOCUMENT DO NOT NECESSARA Y REPRESENT THE OFPICLL POSTIONS OR POLTCIES OP IHE IUNDERS

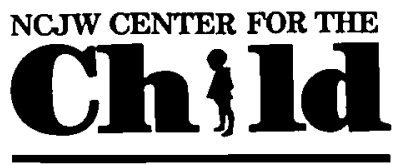

53 West 23rd Street • New York • NY 10010

TEL: (212) 645-4048 • FAX: (212) 645-7466

(C) National Council of Jewish Women, 1997. All rights reserved. 


\section{TABLE OF CONTENTS}

ACKNOWLEDGMENTS $\ldots \ldots \ldots \ldots \ldots \ldots \ldots \ldots \ldots \ldots \ldots \ldots$

PREFACE $\ldots \ldots \ldots \ldots \ldots \ldots \ldots \ldots \ldots \ldots \ldots \ldots \ldots \ldots \ldots \ldots$

INTRODUCTION TO THE PARENT INVOLVEMENT PROJECT $\ldots \ldots \ldots$

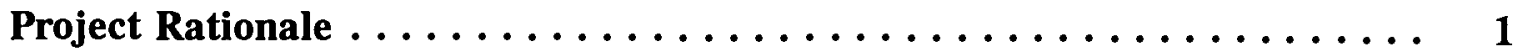

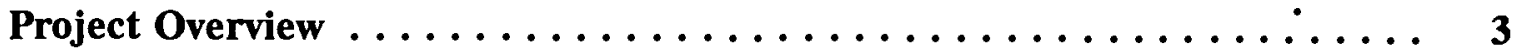

Community Context of Targeted Head Start Agencies $\ldots \ldots \ldots \ldots$

THE LONGITUDINAL STUDY: INTRODUCTION AND

THEORETICAL FRAMEWORK $\ldots \ldots \ldots \ldots \ldots \ldots \ldots \ldots \ldots \ldots \ldots$

Research Questions Addressed by the Longitudinal Study . . . . . . . 10

Conceptual Basis of the Study $\ldots \ldots \ldots \ldots \ldots \ldots \ldots \ldots \ldots$

Conceptualization of Parent Involvement $\ldots \ldots \ldots \ldots \ldots \ldots \ldots \ldots$

Conceptualization of Outcome Domains $\ldots \ldots \ldots \ldots \ldots$

RESEARCH METHODS AND PROCEDURES . . . . . . . . . . . . . . . 17

Overview of Research Design: Strengths and Limitations . . . . . . . . . 17

Sample Recruitment .......................... 22

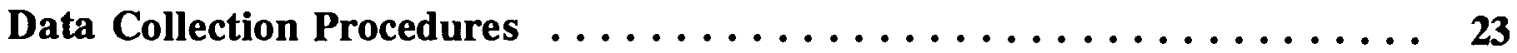

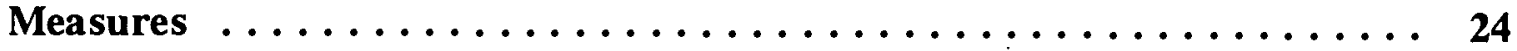

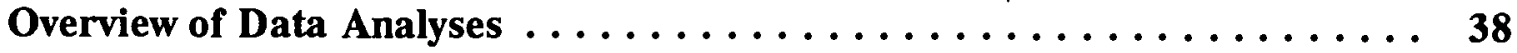

RESULTS: AGENCY ONE . . . . . . . . . . . . . . . . . . 44

Demographic, Contextual, and Personal Characteristics

of Sample at Pre-test . . . . . . . . . . . . . . . . . . . . . . . 44

Changes in Sample Size Over the Course of the Study . . . . . . . . . . 46

Description of Parents' Involvement in Head Start . . . . . . . . . . . 48

Predictors of Parent Involvement $\ldots \ldots \ldots \ldots \ldots \ldots \ldots \ldots$

Parent Involvement and Parent Outcomes . . . . . . . . . . . . . 57

Parent Involvement and Child Outcomes . . . . . . . . . . . . . . 74

Parent Involvement and Older Sibling Outcomes . . . . . . . . . . 80

Utilization of Social Services: Descriptives and Predictors . . . . . . . . 83

Utilization of Social Services and Parent, Head Start Child

and Sibling Outcomes $\ldots \ldots \ldots \ldots \ldots \ldots \ldots \ldots$

Summary and Conclusions . . . . . . . . . . . . . . . . . 89

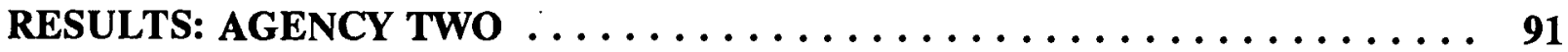

Demographic, Contextual, and Personal Characteristics

of Sample at Pre-test . . . . . . . . . . . . . . . . . . . . 91

Changes in Sample Size Over the Course of the Study . . . . . . . . . . 93

Description of Parents' Involvement in Head Start . . . . . . . . . . . . 94

Predictors of Parent Involvement: Barriers and Facilitators . . . . . . . . 95

Parent Involvement and Parent Outcomes . . . . . . . . . . . . 97

Parent Involvement and Head Start Child Outcomes $\ldots \ldots \ldots \ldots$ 
DISCUSSION . . . . . . . . . . . . . . . . . . . . . . . . . . 104

Summary and Interpretation of Major Findings $\ldots \ldots \ldots \ldots \ldots \ldots$

Additional Methodological Considerations $\ldots \ldots \ldots \ldots \ldots \ldots \ldots \ldots$

Recommendations for Future Research . . . . . . . . . . . . . 121

Policy and Programmatic Implications and Suggestions . . . . . . . . 122

REFERENCES $\ldots \ldots \ldots \ldots \ldots \ldots \ldots \ldots \ldots \ldots \ldots \ldots \ldots \ldots \ldots \ldots \ldots \ldots \ldots$

\section{APPENDLX}




\section{ACKNOWLEDGMENTS}

There are many people who we would like to thank for their help and expertise in conducting this complex research project. First we would like to thank the Head Start Research Group (HSRG) for their hard work, creativity, and support in developing and implementing the Parent Involvement Project. The original group included Blanca Baez, Clare Marie Bonafede, Jane Bradshaw, Beryl Clark, Margaret Dana, Elaine DanavallWilliams, Catherine Faye, Pamela Freeman, Margaret Hernandez, Rochelle Moody, Lenore Peay, John Van Orden, Ruth Robinson, Kiley Roman, Aida Seda, Susan Young and Dr. Edward Zigler. We also would like to thank those who joined the HSRG later in the project, for their assistance.

Next, we would like to thank the parents and children from the two Head Start agencies who participated in the project. Confidentiality precludes naming them personally, but we gratefully acknowledge their contribution.

Dr. Edward Zigler and Elaine Danavall-Williams deserve special mention. As consultants to the HSRG, they freely offered their time and expertise over the course of the project. We are also grateful to Dr. Louis Primavera and Dr. Bernard Gorman for their statistical talents and analytic skills in helping to bring the longitudinal study to completion.

Cynthia Flynn and Hirokazu Yoshikawa are given special thanks. Cynthia, for her undaunted energy'and extraordinary interpersonal ability in carrying out the objectives of the project, and Hiro, for his meticulous and enthusiastic participation.

Chabiram Rampersad's excellent skills, loyalty, and steadfastness in his administrative and fiscal roles throughout the life of the project have been invaluable to its successful completion. Acknowledging him, and his assistant, Nuris Cevallos, for providing administrative support and for producing numerous drafts of the manuscript, including this final report, is especially important.

We also would like to thank Dr. Laura Soden and Jennifer Ritter for their efforts in cleaning and verifying the voluminous amounts of data, gathering information, and contributing to the initial drafts of the manuscripts.

We express our gratitude to the Administration on Children, Youth and Families and the U.S. Department of Health and Human Services for its support of this enormous and worthwhile project. The Federal Project Officers Adrienne Brigmon and Dr. Esther Kresh are thanked for their support and considerable patience over the years.

Finally, we must express our deepest gratitude to a private foundation for their invaluable contribution in allowing us to complete this project and to collect additional data which increases its breadth and relevance.

Without the efforts, contributions, talents and support of all of the above-mentioned, this project would not have been possible. 


\section{PREFACE}

The Head Start Parent Involvement Project has been designed and implemented through a partnership of NCJW Center for the Child and the Head Start community. The commitment to this joint effort is based in the belief that both practitioners and researchers have expertise and creativity that, if combined, will improve the validity of the research design, measures, and results of the study and ultimately increase the probability of the study's findings being utilized. This partnership model of conducting research is consistent with recommendations of the Advisory Committee on Head Start Quality and Expansion to "expand the partnership between research and practitioners by encouraging better communications and better utilization of data" (USDHHS, 1993). Moreover, Edward Zigler has noted that such partnerships play a vital role in the design and implementation of early intervention programs (Zigler \& Finn-Stevenson, 1992). The partnership that was formed for this project was named the "Head Start Research Group" (HSRG), and was comprised of representative Head Start staff and parents, project research staff, as well as consultants from the research and the practitioner communities. The HSRG worked together over several years to refine the design and methods of the studies, select the constructs and measures, develop a specialized intervention for staff and parents, and implement all phases of the project. This report represents the efforts of all members of the group. 


\section{INTRODUCTION TO THE PARENT INVOLVEMENT PROJECT}

Project Rationale

Head Start is a comprehensive, federally funded program for low-income families with young children. Since its inception during the War on Poverty, Head Start has served as a model for the delivery of comprehensive services to preschoolers and their parents. Today, Head Start stands as the first "two-generation" intervention, combining supports for children's well-being and early development, while also seeking to ameliorate family poverty and increase the dignity and self-worth of family members (Parker, Piotrkowski, Horn \& Greene, 1995). During Fiscal Year 1994, Head Start served an estimated 740,493 children and their families nationwide, with a budget of $\$ 3,325,728,000$. New York State served an estimated 39,062 of those children and their families, with a budget of $\$ 215,678,102$ (USDHHS, February, 1995).

Much attention traditionally has focused on Head Start's effectiveness on children's IQ, cognitive development, and subsequent educational attainment. In part, this has resulted from the "naive" environmentalism of the 1960's that viewed Head Start as a "quick fix" intervention to raise children's IQ and make them more successful at school, breaking the "cycle of poverty" passed down from generation to generation (Valentine \& Stark, 1979). However, from its inception, Head Start was designed to focus on environmental enrichment for the "whole child," encompassing his/her cognitive, language, health, nutritional, and socioemotional needs within the context of strong family support and involvement. Head Start's mandate calls for "maximum feasible participation" of parents in all programmatic efforts (USDHHS, 1970). Parents were included in all aspects of the program, including program planning, management, and implementation; and volunteering in the classroom, office, and kitchen. They also were involved in parent education experiences, social activities, and were given support through the provision of social services (USDHHS, 1970; USDHHS, 1975). Edward Zigler, who was instrumental in the founding and development of Head Start, views parents as the vital force behind long lasting effects on children (Zigler, 1978; Zigler \& Valentine, 1979; Zigler \& Muenchow, 1992; Zigler \& Styfco, 1993a). 
Although parent involvement in Head Start is an integral and mandated component of the Head Start model, it has received scant attention from policy makers and researchers. Even so, anecdotal evidence abounds on the positive impact of Head Start on parents (e.g., USDHHS, September, 1979; CSR, Inc., January, 1990; Replogle, 1995). In addition, Head Start programs, through their annual Program Information Reports (PIR's), are required to report the extent of parent involvement in their programs. Yet, there currently exists little solid research regarding the effects on families of parent involvement. Instead, it generally has been assumed that parent involvement in Head Start plays an important positive role for both parents and children.

Most of the studies that have examined the effects of parent involvement on families defined parent involvement in terms of the impact on children's development of a special experimental intervention of narrow content and short duration (see Leik and Chalkley, 1988; McKey, et al., 1985; USDHHS, 1996). Only a few have studied parent involvement as it normally and naturally occurs, envisioning it as all of the activities and experiences for parents over the course of a Head Start year. Of those, an even smaller number have studied its effects on the parents themselves and other family members, such as siblings (Lindsey, 1987; McKey et al., 1985; Oyemade \& Washington, 1985; Oyemade, Washington \& Gullo, 1989; Parker, Piotrkowski \& Peay, 1987; Slaughter, Lindsey, Nakagawa \& Kuehne, 1989). Most of those studies used a cross-sectional design, and the parent involvement data were collected retrospectively. Parker, Piotrkowski and Peay (1987) improved on that research with a longitudinal study of the effects of parent involvement on parents' personal well-being. The normally and naturally occurring parents' participation in Head Start over the course of a Head Start year was measured. That sample was small, however, and the impact of parent involvement on a full spectrum of parent, Head Start child, or sibling variables were not examined.

By ignoring the potential benefits of parent participation for parents themselves and other family members, the full benefits of Head Start may be seriously underestimated (see also Slaughter et al., 1989). Moreover, Congress recently enacted legislation that called for revitalizing Head Start by developing revised Performance Standards and measures to improve quality and streamline the transition from Head Start to public school (Head Start Act, as amended May 18, 1994) while other social programs are being severely cut or 
eliminated. The prospect of an expansion of Head Start services with matching increases in funding has triggered a new round of debates in Congress and the media regarding Head Start's effectiveness, specifically, whether or not Head Start produces tangible benefits that justify the investment of substantial federal funds.

What has been missing thus far is a more comprehensive assessment of naturallyoccurring parent involvement in Head Start that would demonstrate the impact of this important component of the program on parents, Head Start children and siblings. Demonstrating benefits of Head Start beyond the Head Start child's cognitive development could clearly have wide-ranging implications for policy and practice.

In addition, such research could help Head Start rethink its parent involvement component in light of the increasingly stressful socioeconomic climate in which economically-disadvantaged families now live. Creating additional ways to understand, reach, and engage parents would enhance Head Start's mandate for becoming a fully developed, "two-generation" intervention program for young children and their families.

\section{Project Overview}

To help address these issues, in 1990, the National Council of Jewish Women Center for the Child in collaboration with the Yale Bush Center in Child Development launched the Parent Involvement Project. This endeavor included a longitudinal study of parent involvement in Head Start and its impact on parents, Head Start children, and their siblings. Two Head Start agencies served as research sites and were partners in the planning and execution of the project. Initial funding was received from the Administration on Children, Youth and Families (ACYF) and the National Council of Jewish Women. Additional funding from the Smith-Richardson Foundation made possible the completion of the project, and allowed us to increase its scope and relevance by enabling a follow-up study of the Head Start children and their families into elementary school.

This longitudinal study of parent involvement in Head Start had two major objectives:

1. To identify through a longitudinal study the "predictor" variables associated with parents' participation in Head Start.

2. To examine the relationship between parents' participation in Head Start and outcomes for parents, their Head Start children, and siblings. 


\section{Community Context of Targeted Head Start Agencies}

In order to fully understand and interpret our findings, we developed a community profile compiled from existing available data ${ }^{1}$ on variables relevant to our sample and research questions. Information regarding how the project sample differs from the larger community guides our understanding of to whom we can generalize the findings. Similarly, such information could be used to explain unusual or counter intuitive patterns among the variables.

\section{Community Profile - Agency One}

\section{Culture and Ethnicity}

According to 1990 U.S. census data, the Agency One catchment area has become predominantly a Latino immigrant community, two-thirds of which is now Dominican (African American [Black]: 12\%; Euro-American [White]: 18\%; Puerto Rican and other Latino groups excluding Dominicans [Latinos]: 12\%). In addition, the U.S. Immigration Naturalization Service reported that nearly half $(47 \%)$ of these immigrants have arrived during the past decade, and only $32 \%$ were naturalized Americans in 1990 . Given this, it is not surprising that half the residents of the community speak little or no English, and $65 \%$ reported that they speak only Spanish at home.

\section{Income Level and Poverty}

For this community as a whole, poverty has tripled in the past 20 years, which most likely is due to the influx of Dominican immigrants. Specifically, while the per capita income for the overall community was $\$ 13,000$ in 1990 , median income for Dominican families was $\$ 6,600$ (i.e., $41 \%$ at or below poverty level); for Latinos, median income was $\$ 11,300$ (approximately 30\% below poverty level); for African-Americans, average income was $\$ 14,500$ ( $28 \%$ below poverty level); and for Whites, $\$ 21,333$ (only $15 \%$ below poverty level). Unemployment for Dominicans in this neighborhood was highest in comparison to the other groups as well: Latinos and African-Americans reported similar unemployment

\footnotetext{
1 The information for the community profile comes from the following sources: 1) NYC Department of Health, 1988; 1990 US Census; NYC Housing and Vacancy Survey; 1992 NYPD Annual Statistical Report; NYPD Annual Statistical Reports, 1989-1991; cited in Garfield \& Abramson, 1994. 2) Ft. George Community Enrichment Center, Inc., Head Start Community Needs Assessment, prepared by Floyd D. Page, 1/31/94.
} 
rates of approximately $4.5 \%$; Whites reported only $4 \%$; but Dominicans were highest at $9 \%$ unemployment.

\section{Education}

Educational level for adults 25 years of age or older within the community overall, was two thirds (66\%) high school graduates, $25 \%$ of whom were also college graduates in 1990. However, breakdown by ethnic/cultural group reflected considerable inequality: whereas $90 \%$ of Whites had completed high school, $51 \%$ of whom had finished college, only $70 \%$ of African-Americans were high school graduates and $42 \%$ had completed college. The immigrant groups' educational level was even lower than that of African-Americans: only half of Latinos were high school graduates and $26 \%$ of these completed college; the majority cultural group, Dominicans, had received the least education: only $44 \%$ had completed high school and 23\% of these were college-educated in 1990.

\section{Family Composition}

The size and configuration of individual households in this community varied considerably by ethnicity/cultural group. To begin with, while the average number of residents per household community-wide is three persons (also the average for Latinos), for both African-Americans and Whites, the average size is approximately two persons (2.1 and 1.8, respectively), but for Dominicans, households are largest: four persons per household on average. Forty-four per cent of these Dominican homes are headed by women, compared to only $25 \%$ of other Latinos, $23 \%$ of African-Americans, and $7 \%$ of Whites. Hence, even though Dominican and other Latin women were more likely to have been married at some point (75\%) compared to either African-Americans (58\%) or Whites (59\%), they were more likely to be both raising larger families, and doing so without a partner. Finally, these single mothers as a group tend to be younger than their counterparts in the other groups.

According to United Hospital Fund data (1988), the community-wide birth rate was higher (50\%) than in the city as a whole in 1988 , but the infant mortality rate, 8.6 deaths for every 1,000 births, was lower. This is in spite of $20 \%$ of mothers who receive little or no prenatal care, and $18 \%$ of births that are high-risk (premature or low birth-weight), compared to only $10 \%$ in the city as a whole. 


\section{Quality of Life: Home and Neighborhood}

In the community of Agency One, almost all residents (97\%) rent their living quarters. Approximately half the population of the community claimed to be well-housed. However, only $40 \%$ reported that their quarters had no deficiencies (i.e., needed repairs, insufficient space, or inadequate utilities), and nearly $20 \%$ reported three or more deficiencies. These figures can be compared to those of a nearby middle-class area, where $60 \%$ of residents reported no deficiencies and only $8 \%$ reported three or more. In addition, $11 \%$ of community residents reported lacking a telephone. Once again, the Dominican group had the greatest difficulty at $20 \%$ without a phone; approximately $10 \%$ of both African-Americans and Latinos reported being without telephones; and only $2.5 \%$ of Whites were without this service. Additionally, the infrastructure of the area is among the oldest in the city, and its incidence of lead-poisoning is in the highest third.

The neighborhood surrounding Agency One is reported to be among the most dangerous in the city. For example, in 1992, 50 murders, 21 rapes, 487 assaults, and 772 robberies were reported for every 100,000 residents. Compared to a nearby middle-class community, all crime statistics are higher. They do not exceed those for the city as a whole, except for the death-by-homicide rate, which is considerably higher, especially among young men. Related to this high homicide rate is the existence of a pervasive and violent underground drug economy that is common knowledge, but for which exact figures are unavailable. Nevertheless, the community lacks adequate police protection, as only one precinct serves the whole area compared with other districts half the size that are policed by two precincts.

\section{Community Profile - Agency Two}

\section{Culture and Ethnicity.}

The Agency Two catchment area is large and relatively diverse in terms of ethnicity and SES, with African-American, Asian, White, Middle-Eastern, Native American, and Latino groups all represented. However, the majority culture is clearly Euro-American (approximately $90 \%$ ) and native-born (88\%). In addition, all but $19 \%$ of the residents of this district speak English, 6\% reported that they speak Spanish, and 3\% speak an Asian or Pacific Island language at home. 


\section{Income Levels and Poverty}

In 1990 the median annual household income for the district as a whole was $\$ 43,861$, with $73 \%$ claiming an income of above $\$ 25,000$ per year. The median family income was $\$ 50,664$ and $81 \%$ of families reported an income of over $\$ 25,000$. Also for the majority of households $(81 \%)$, wages or salary were the income source. In $1990,5 \%$ of males in the work force over 16 years of age were unemployed; $3 \%$ of women in this category were unemployed.

Within this emerging picture of a nearly homogeneous White, middle-class district, pockets of poverty co-existed: approximately $11 \%$ of households and $6 \%$ of families reported income at or below the poverty level. Additional figures for this group illustrate increasing poverty related to larger families and single parenthood: families with children under 18 years of age, $10 \%$ in poverty; families with children under five years of age, $12 \%$ in poverty; families headed by a single female, $24 \%$ in poverty; with children under 18 and five years of age, $38 \%$ and $41 \%$ in poverty respectively. For only $6 \%$ of households was some or all income derived from public assistance.

\section{Education}

Educational attainment in this middle-class community was relatively high. Of adults over 25 years of age, $77 \%$ had finished high school and $21 \%$ of these completed college. However, another fifth of adults (21\%) dropped-out of school during high school or before (7\%).

\section{Family Composition}

Of the nearly 100,000 families living in the Agency Two catchment area, only $4 \%$ are headed by a single female. Otherwise, families with children under 18 years of age in the area tended to be headed by adults with partners. Hence, while families headed by single mothers were three times as likely to live in poverty, they comprised a small proportion of the total population.

\section{Quality of Life: Home and Neighborhood}

One indicator of neighborhood stability is the fact that more than half of area householders (58\%) had lived at their address for five years or more in 1990 . Forty-seven per cent of families in the Agency Two catchment area lived in homes they owned. Of these, total housing expenses exceeded a third of their income for only $18 \%$; more 
commonly (65\%) housing costs accounted for only a quarter of the total income. Renters in the area paid a higher proportion of their income for housing: $35 \%$ paid over a third of their income; half (55\%) paid under a quarter of total income. Overall, the median gross monthly payment for housing was $\$ 578$ per household; only $3 \%$ paid more than $\$ 1,000$. Nearly all (97\%) households in the area reported having telephones and $82 \%$ of families and/or households own a car.

The crime rate in this middle-class area appears to be moderate, compared to statistics for the city as a whole, e.g., (NY Times, 7/8/95). In one precinct serving a typical neighborhood of this district, the police department logged 1 reported murder, 31 cases of manslaughter, 14 rapes, 320 robberies, 297 assaults, 1358 burglaries, and 2839 thefts of automobiles in a population of 114,000, during 1994 .

\section{Agency Profiles}

Agency One is a center-based model that was established in 1981 with new Head Start expansion money, having been designated a low-income community by the city in 1980 . This Head Start agency served as the location for an earlier study (Parker et al., 1987). The agency has two centers within a short walk from each other. Its catchment area is primarily Latino. When the study began in 1990, the agency had an enrollment of 294 children. It had six classrooms, all with half-day enrollments (9:00 am--noon; or 12:30 pm--3:30 pm). By 1991 (Cohort I), the enrollment at the agency had increased to 321 with an added fullday classroom. By 1992 (Cohort II), it was operating with six half-day and two full-day classrooms with a total enrollment of 348 children.

Agency Two has a standard center-based program with part-day and full-day enrollment. Established in 1966 as one of the original Head Start agencies, it was based at two locations (since the completion of data collection, a third center has been added). One center is full-day (9:00am--2:45pm) located in a middle-income neighborhood. Children are bussed from poverty pockets throughout the larger community, where the families are predominantly African-American and low-income. The other center is located in a lowincome community, and has part-day enrollment (9:00am--Noon; 12:30pm--3:30pm). Children are drawn from three geographic areas, communities which are predominantly 
African-American and low-income. In 1990, 151 children were enrolled. By 1991-1992, the enrollment increased to 179. In 1992-1993 the enrollment decreased somewhat to 168 .

The following information about Agency One and Two comes from their 1991-1992 and 1992-1993 Program Information Reports (PIR), an instrument used by the Administration for Children and Families to monitor the compliance of the agency with Head Start Performance Standard (USDHHS, 1984).

At Agency One, almost all of the children served were Latino, of Dominican origin (99.6\%). The majority were four-year-olds (62\%). Most families were below the poverty level (98\%) with 95\% receiving AFDC benefits. In 1991-1992 and 1992-1993, all parents volunteered at least once. In 1991-1992, parent involvement hours was distributed as follows: classroom volunteering (284), policy council (36), fundraising (270) and other (78). Parent involvement in 1992 - 1993 included: classroom volunteering (320), policy council (27), fundraising (267) and other (87).

At Agency Two, over three-quarters of the children were four-year-olds (79\%). At the bussed center $(n=67) 56.7 \%$ were African-Americans, $16.4 \%$ were Latinos and $26.9 \%$ were white. At the other center $(n=84) 78.6 \%$ were African-Americans, $13.1 \%$ were Latinos and the remainder were white. All families were below the poverty line. Parent involvement was generally high, with nine out of ten parents participating in the program. In 1991-1992 and 1992-1993, all parents volunteered at the agency at least once. Parent involvement were distributed as follows for 1991-1992: classroom volunteering (22), policy council (24), fundraising (75) and other (95). In 1992-1993 the distribution was: classroom volunteering (33), policy council (24), fundraising (73) and other (124).

Both Head Start agencies follow the guidelines for parent participation and documentation outlined in the Handbook for Involving Parents in Head Start (USDHHS, 1980), although the actual implementation of their parent involvement program reflects the unique qualities of their program, their staff and their parents. Both Head Starts are considered well-run and effective by their grantee and regional oversight office (Region II). Both were designated for expansion funding for September 1990 to accommodate an additional 60 families each. 


\section{THE LONGITUDINAL STUDY: \\ INTRODUCTION AND THEORETICAL FRAMEWORK}

Research Questions Addressed by the Longitudinal Study

Listed below are the first and second project objectives and the specific research questions to address them:

Objective 1: To identify through a longitudinal study, the "predictor" variables associated with parents' participation in Head Start

- What are the demographic, contextual, and personal variables related to parent participation in Head Start?

Objective 2: To examine the relationship between parent participation in Head Start and the parents themselves, the Head Start children, and siblings

- Is parent participation in Head Start positively related to positive parent-child relationships?

- Is parent participation in Head Start positively related to the home learning environment?

- Is parent participation in Head Start positively related to parents' well-being?

- Is parent participation in Head Start positively related to parents' skills and behaviors that promote socioeconomic self-sufficiency?

- Is parent participation in Head Start positively related to parents' involvement in their Head Start child's education in elementary school?

- Is parent participation in Head Start positively related to the Head Start child's social competence at the end of the Head Start year and at the end of kindergarten?

- Is parent participation in Head Start positively related to the social competence of an older sibling of the Head Start child? 


\section{Conceptual Basis of the Study}

Head Start was developed to be a two-generation program that addresses the needs of both children and their parents. This two-generation emphasis is clearly incorporated into the Head Start Performance Standards. Parents are to be involved in all aspects of Head Start. Evaluations of the impact and benefit of Head Start have been conducted since the program's inception. However, these studies have largely ignored the parent component, resulting in a possible underestimation of the benefits of Head Start for both the parent and the child. As Zigler (e.g., Zigler, 1978; Zigler \& Berman, 1983; Zigler \& Muenchow, 1992) has frequently noted, evaluations of Head Start should go beyond the individual child to include an assessment of the impact of the program on parents of the children in the program as well. The present study was developed to address this issue and contributes to a fuller understanding of the benefits of Head Start in three important ways: (1) by assessing the benefits of parents' participation in Head Start on the parent, the Head Start child, and an older sibling; (2) by examining in detail the full range of naturally occurring parent participation, relating different types of participation to a variety of outcomes; and (3) by exploring the demographic, contextual and family characteristics that may be associated with (i.e., or "predict") the extent of parents' participation in the program.

Reviews of Head Start research over the last two decades point to the paucity of rigorous efforts to understand parents' participation in Head Start, and its impact on the parents themselves and on their Head Start child (see, for example, Hubbell, 1983; Collins \& Kinney, 1989; McKey et al., 1985; USDHHS, 1996). Regardless, the limited empirical evidence available, as well as much anecdotal information, suggest that parents' participation in Head Start can transform their lives as well as the lives of their children.

Most early studies of the effects of parent involvement in Head Start focused on its impact on the Head Start child, specifically, aspects of the child's functioning or learning (see bibliographic index of Zigler \& Valentine, 1979). A comparatively small number of studies included an interest in changes in the parents themselves (e.g., Adkins, 1971; Boger, 1969; Clarizio, 1968; Gordon, 1969; Jacobs, 1970; Swift, 1968). These early investigations, however, suffered from numerous design and measurement problems, including inadequate 
measures of involvement, small sample sizes, and lack of reliable outcome measures. One national study of 20 Head Start centers by Midco Associates (Bromley, 1972) took into account several of the limitations of the earlier work and found that parent involvement was linked to parents' personal growth and change. Their cross-sectional design, use of retrospective self-reports by parents, and the non-independent measures of involvement still limited the conclusions one could draw from that report.

Parker, Piotrkowski and Peay (1987) attempted to address some of these limitations by including pretest and posttest data in their study. Moreover, each mothers' participation in Head Start was measured objectively, rather than through self-report. They found that mothers who participated more (high and medium involvement) reported greater well-being at the end of the program year than mothers who participated less (low involvement). The effect of parent participation was hypothesized to be due to the practical and psychological support offered by the Head Start program. This support may have enhanced mothers' well being because it reduced the life stresses associated with poverty, while also offering comfort. Although the existence of pretest data allowed stronger inferences to be drawn about the impact of participation, the simple frequency count of involvement'activities did not consider how different types of participation were related to different parent outcomes or how parent involvement might affect various child ou tcomes.

More recent research that has examined the impact of parent involvement on parents themselves has had similar methodological and measurement limitations as the earlier studies. An exploratory study by Slaughter, Lindsey, Nakayawa, and Kuehne (1989) found that more involved Head Start mothers had higher levels of self-esteem and ego development than less involved mothers. However, the cross-sectional design, with lack of pretest data, made it difficult to determine whether the greater self-esteem and ego development were the result of participation per se or due to preexisting differences among the parents. Oyemade, Washington, and Gullo (1989) found that parents from Head Start centers with high involvement were more economically self-sufficient (i.e., more likely to be above the poverty level) when followed up than parents who had attended low-involvement centers. In addition, they found that the high-involved parents were significantly different from the low-involved parents on family income and employment of the mother and the father in the expected direction at the end of Head Start. A major limitation of that study, 
however, was the way in which parent involvement was measured. Interviews were conducted with parents several years after their Head Start experience. This use of retrospective information makes the parent involvement data less reliable and valid.

Other researchers have explored factors that might predict patterns of parent involvement. Education, mothers' age, and number of years in Head Start have been found to predict involvement (Slaughter et al., 1989; Oyemade et al., 1989). A study carried out by the Department of Health, Education and Welfare (HEW Region III, 1977) found that barriers to participation included having younger children at home, attending school, and working. Parker, Piotrkowski and Peay (1987) found that quality of housing predicted frequency of involvement. These studies, however, did not examine barriers as they related to different conceptualizations of parent involvement (i.e., frequency and content type).

The present study was initiated because of the clear need to more rigorously understand parent involvement in Head Start. It builds on previous work and extends it in several ways. First, pretest measures are used to control for initial differences in the outcomes of interest. Second, objective measures of parent involvement are used that assess multiple dimensions of parental activities and experiences in the program. Third, a wide array of outcomes are considered that assess the effects of parent involvement on parents and children. Fourth, the study design is longitudinal, following the Head Start children into elementary school, to assess the longer-range effects of Head Start on parents' involvement in their children's education and efforts toward self-sufficiency and children's relationships with others. Fifth, the impact of parent involvement on an additional family member, an older sibling, is examined.

Finally, this project moves the field forward by attempting to understand what characteristics and circumstances of parents and children predict parent involvement. In this longitudinal study, we examined the role of a wide range of demographic, personal, and contextual characteristics of the parent, Head Start child, and older sibling in predicting parents' participation. The longitudinal study uses a large sample and assesses multiple types of parent participation. A variety of predictors, including personal, family, and 
community factors were assessed. These analyses addressed the present study's Objective $1 .^{2}$

\section{Conceptualization of Parent Involvement}

Parent involvement in Head Start is the major independent variable in this study. There were several ways we could have conceptualized and measured this variable. For example, Valentine and Stark (1979) have conceptualized parent involvement as parent education, parent participation in decision-making, and parent control. Bromley (1972). utilized the concepts of parent as "learner" and "decision-maker." Slaughter et al., (1989) identified three levels of complexity of parent involvement, with decision-maker being the highest. Parker, Piotrkowski, and Peay (1987) used the operational definition from the Head Start Performance Standards (USDHHS, 1975; 1984), that is, all of the family support and parent participation activities and experiences offered over the course of the Head Start year. In addition, they included the practical and psychological support functions of Head Start, offered in part through the Social Services component.

Building on the variety of ways parent involvement has been conceptualized and on our previous work (Parker, Piotrkowski, \& Peay, 1987), two ways of conceptualizing involvement have been identified for this study. First, parent involvement is conceptualized (and operationalized) as a simple amount (each activity is weighted by number of hours spent on it), of all of the parent's activities and experiences in the Head Start program (See Parker et al., 1987). Thus, more involvement is hypothesized to be related to more positive outcomes for parents and children.

However, amount of parents' involvement in Head Start without regard to type of activity may be inadequate to capture the complexity of parents' experiences. Four general types of activities differing in content were identified by the project's Head Start Research Group: 1) volunteering in the program, primarily in the classroom, but also in the office, in the kitchen and on field trips; 2) workshops, covering such topics as parenting, food preparation, and nutrition; 3 ) attending and participating in policy-making committees and meetings, including such activities as class committee meetings, delegate agency committee

\footnotetext{
${ }^{2}$ Objective 1: To Identify through a longitudinal study the "predictor" variables associated with parents' participation in Head Start.
} 
meetings, and personnel practices committee meetings; and 4) social services, includes parent contacts with the Social Services staff pertain to personal problems or issues; concerns about the Head Start child, another child in the family, and/or another family member; housing or financial problems; immigration issues; and community resource referrals. These four types of involvement activities are reflected in the Head Start Performance Standards.

Objective $2^{3}$ was tested by examining the relationship of amount of parent involvement -- without regard to type of activity -- to positive outcomes for parents, the Head Start child, and his/her older sibling. The relationships between specific types of involvement activities and these outcomes were explored as well.

\section{Conceptualization of Outcome Domains}

Consistent with Head Start's stated objectives and Zigler and Trickett's (1978) argument that increased social competence should be considered the major outcome of successful intervention programs for children, "social competence" is the major outcome of the study. Zigler and Trickett defined social competence as having two key components: 1) successfully meeting society's expectations, and 2) personal development and selfactualization. In this conceptualization, the social competence construct may be meaningfully applied to parents participating in an intervention program like Head Start, as well as to their children. Because this concept of social competence is broad and multifaceted, like other researchers, we will focus on those aspects of social competence most likely to be affected by parent involvement in Head Start.

Specifically, we hypothesized that parent involvement in Head Start may enhance parents' social competence by providing them with supportive services and support to reduce life stress, by enhancing skills that promote the confidence to try new behaviors, and by facilitating parent's feelings of efficacy and control over their own lives. Enhanced social competence may extend to the parent-child relationship in the form of increased feelings of parenting competence, new understandings of child development, and improved methods of interaction between parent and child. In addition, parent involvement could enhance how

\footnotetext{
${ }^{3}$ Objective 2: To examine the relationship between parents' participation in Head Start and the parents themselves, the Head Start child, and siblings.
} 
parents deal with other social institutions, such as schools. These new understandings and behaviors could transfer to how the parent views their child's school environment, and to new ways of interacting with school staff. Thus, we would expect highly involved Head Start parents also to be more involved in their children's education and school experience.

Thus, for participating parents, we focused on the following indicators of social competence: 1) positive parent-child relationships; 2) an enhanced home learning environment; 3) subjective well-being (i.e., feelings of mastery/control and a lack of depressive symptomatology); 4) skills and behaviors that promote socioeconomic selfsufficiency (e.g., paid employment, job training/education, and not being pregnant during the follow-up year); and 5) involvement in the Head Start child's education in kindergarten.

In addition to the evidence that parent participation in Head Start is related to positive outcomes for parents, parental involvement in all aspects of Head Start may have benefits for children as well (in addition to the direct benefits children receive from their center-based experience in Head Start). The present study hypothesizes that parents who are more involved in Head Start will gain skills, behaviors, and attitudes that result in improved outcomes for their Head Start child. There are many potential ways in which parent involvement in Head Start could be beneficial to children. Volunteering in the Head Start child's classroom may provide the parent with information about her child's schoolreadiness, as well as instructional techniques with which to help her child improve these skills. Attending parenting workshops may result in improved attitudes towards childrearing and in a more positive view of young children's normative skills and behaviors. Parents' attendance at policy meetings and participation in decision-making and leadership-building activities may result in the parent's being a more effective role-model for the Head Start child. Thus, being more involved in all aspects of Head Start should provide positive benefits for the Head Start child's own social competence affected through changes in the parent.

In this study, we examine the relationship between parent involvement in Head Start and positive outcomes for the Head Start child. For the Head Start child we focus on the following indicators of social competence at the end of the Head Start year: 1) school 
readiness; and 2) positive normative social skills and behaviors at home. Head Start child outcomes one year after the Head Start experience include teacher ratings of:

1) the Head Start child's adaptation to the kindergarten classroom, and 2) current and anticipated school performance.

For the Head Start child's older sibling (see Rationale for Studying Older Siblings below), we focus on the following indicators of social competence: 1) positive adaptation to school; 2) positive peer relations; 3) overall social competence; and 4) other indicators of school success, including appropriate grade level and not being placed in special education.

\section{RESEARCH METHODS AND PROCEDURES}

\section{Overview of Research Design: Strengths and Limitations}

The original design for the longitudinal study was a quasi-experimental pretest posttest design with two cohorts (Cook \& Campbell, 1979) at each of two Head Start agencies in a large urban city. The agencies each had two centers that participated in the study. Cohort I children attended Head Start from September, 1991 through June, 1992. Cohort I families whose children attended kindergarten from September, 1992 - June, 1993 comprised the sample that was followed up one year later (i.e., kindergarten follow up). ${ }^{4}$ Cohort II children attended Head Start from September, 1992 through June, 1993. Cohort I older siblings (ages 5-12) in the study attended elementary school from September 1992 June, 1993 and were followed up as part of the Cohort I kindergarten follow-up of the family.

\section{Across Cohort Comparisons}

Initially, the design called for within-agency comparisons across cohorts, based on the assumption that the cohorts would be comparable on characteristics important to the interpretation of the findings of the study. This assumption seemed reasonable because traditionally the Head Start programs at the research sites and the catchment areas were stable and similar from year to year, a necessary requirement for cohort comparisons (Cook

\footnotetext{
${ }^{4}$ Not all Cohort I children attended kindergarten in the fall of 1992 because some were too young.
} 
\& Campbell, 1979). However, between June, 1992 and September, 1992, the Job Opportunities and Basic Skills (JOBS) welfare-to-work legislation (1988) was implemented in the city where the project was being conducted and dramatically affected the Head Start programs. In Head Start year 1991-1992 (Cohort I), families receiving some form of Aid to Families with Dependent Children (99\% of the sample) were not required to participate in job training or educational programs to keep their financial benefits, whereas, in Head Start year 1992-1993 (Cohort II), many of the families were mandated to do so. Naturally, participating in job training and educational programs outside of Head Start could take up time that otherwise might be used for involvement in Head Start activities and experiences.

This major policy initiative affected the study in two important ways. First, Cohort I and Cohort II became non-comparable on the major variable of the study, parent involvement, making combining cohorts no longer feasible. Second, Cohort II outcome analyses could not be conducted as planned in the absence of key data regarding parents' participation in welfare-to-work programs during the Head Start year. ${ }^{5}$ Therefore, the design representing the data reported here is a one group pretest-posttest passive observational design (Cook \& Campbell, 1979). Because there was individual variation in extent and type of parents' participation, this design permits the analysis of associations between extent of involvement and outcome scores while controlling for initial differences in the outcome and for important demographic differences (e.g., mother's age, education).

\section{Between Agency Comparisons within Cohorts}

The design originally also called for between agency comparisons within each cohort. In order to make these comparisons, sites ought to be similar on the major variable being studied, again, parent involvement. It became apparent, however, that the parent involvement component at each site was defined and implemented quite differently. These differences seemed to be a product of several factors including cultural variation, the style and philosophy of the administrative staff, and the nature of the community sponsoring

\footnotetext{
${ }^{5}$ When it became apparent to research staff that a considerable subgroup of Cohort II families were involved in mandatory job-training and education programs, we incorporated questions about this at posttest. However, most mothers were not able to accurately report on the extent or actual name of the programs in which they were involved. Therefore, for this study, we present outcome data for Cohort I only.
} 
board. Thus, statistical comparisons between the two agencies were not warranted, and consequently we chose to analyze all data separately by agency.

\section{Absence of a Control Group}

Another design consideration was whether to include a group of non-Head Start parents and children as a comparison group to determine the effects of parent involvement in Head Start. There were several reasons this was not done. First, it is difficult to find appropriate community comparisons for Head Start families and problems of interpretation arise from using noncomparable comparison groups. For example, Lee, Brooks-Gunn and Schnur (1988) found that Head Start families tended to be worse off than comparison families, a situation that can result in misleading findings.

Second, we chose not to treat parent involvement as a monolithic experience (i.e., involved/not involved) because there is considerable natural variation in it -- from parents who are minimally involved to those who are highly involved -- and we were interested in the effects of this variation (Parker et al., 1987). A simple comparison of parents in Head Start with those not in Head Start could easily obscure these within-group variations. The benefit of the design we chose is that we can examine the effects of naturally-occurring variations in parent involvement within each cohort. Because all families have selected themselves into Head Start, we eliminated potential unaccounted differences between community comparisons and Head Start families.

\section{Issues of Causality}

Our design does not allow for inferences of causal direction because the effects of self-selection are not eliminated. Although all families have selected themselves into Head Start, there are most likely personal, demographic, and contextual factors that account for differing initial pretest scores on the outcomes of interest which may result in differences in outcomes regardless of the impact of parent involvement. We have attempted to deal with the problem of causality, in part, by selecting a set of demographic variables which theoretically may be related to the outcomes of interest and by controlling for these variables. Although this approach is not ideal because a non-measured variable(s) could 
still be "causing" relationships between parent involvement and the outcomes, it nonetheless is an important step forward from cross-sectional studies of parent involvement.

\section{Issues of Generalizability}

Because of limits in funding, the study has focused on only two Head Start agencies (four centers) in a large northeastern city. The question of representativeness of the agencies has several components. First is the issue of generalizability of findings from two agencies to the population of Head Start agencies across the country. Because of the paucity of longitudinal research on the effects of parent involvement in Head Start, even a relatively small scale study such as this one can make significant empirical and theoretical contributions. In fact, in Head Start Research and Evaluation: A Blueprint for the Future (Collins, Management Consulting, Inc., 1990), Collins suggests that important contributions can be made by several smaller studies that collectively contribute to a body of knowledge. This study can help fill an important void and can provide vital information to policy makers that is now lacking. Moreover, by determining how the parent involvement program operates in a few Head Start agencies, the study can help refine research questions and open the door to more extensive studies based on a cross-section of representative agencies.

A second generalizability issue pertains to differences in populations served by the two Head Start agencies in the study. One agency serves a largely Dominican immigrant population; the other agency serves primarily African American, Hispanic, and Caucasian families. We anticipate that there will be similarities and differences in the processes underlying parent involvement for these different populations. Since the data will be analyzed separately by agency, similarities across the different populations will strengthen our ability to generalize.

A third issue relates to the uniqueness of Head Start agencies and their programs. It is common in the Head Start community to think of each Head Start agency as being unique. However, despite differences among Head Start programs, there is considerable uniformity among them that stems from adherence to the Head Start Performance Standards, the common criteria for hiring staff and for training, and from the commonalities among programming content (e.g., Exploring Parenting, USDHHS, 1976; Looking at Life, 
USDHHS, 1987; and recent federal mandates for literacy, job training, and substance use prevention programs, Horn, 1993;).

The two agencies chosen to participate in the research study were selected because they were considered typical Head Starts by their grantee and regional oversight office: they had not received funds for specialized programs (e.g., focusing on special needs children); they were not demonstrations; they each had both half-day and full-day classes; and their Program Information Reports (PIRS) had been in compliance for many years. Moreover, their grantee and regional office consider these Head Start agencies of "good," rather than of "weak" quality. ${ }^{6}$

A fourth issue is whether the Head Start context in this particular northeastern city is different from that of other communities. We believe that the city shares much in common with other large, urban communities in terms of the populations served (immigrant non-English-speaking, African American, etc.) and in terms of the types of problems poor people in general face, such as extreme poverty, substance use or contact, crime, and poor housing. One of the agencies is located in an inner city immigrant community, whereas the second agency is in a smaller, nonimmigrant community where transportation is a problem for some families. Thus, the Head Start agencies in this study most likely share many features with other urban Head Start centers. Their populations and community contexts do differ from rural Head Starts and those in small towns. Consequently, the study findings would most likely generalize to urban Head Start agencies.

\section{Rationale for Studying Older Siblings}

Older siblings of Head Start children were chosen for this study, specifically siblings between the ages of five and twelve. While some studies have addressed the effects of a family's participation in Head Start on younger siblings, at the time that this study was launched, little was known about the impact of parents' participation in Head Start on the development and competence of older siblings of the Head Start child. It was thought that this group of children might be affected by potential changes in their parents' child-rearing

\footnotetext{
${ }^{6}$ Personal communications, Agency for Child Development (ACD) Administrator and Agency for Children and Families (ACF), Region II Administrator, 1990.
} 
attitudes and feelings of personal well-being as well as potential changes in the family's home learning environment, as a result of the parents' participation in Head Start. The 12year-old cut-off age was selected because of the probability of peer-dominated effects in the teenage years.

\section{Sample Recruitment}

Two-hundred and three mothers out of a possible 256 agreed to participate at Agency One (79\%) and 112 mothers out of a possible 151 agreed to participate at Agency Two (74\%). Parents were recruited for the longitudinal study in several ways. First, the research staff presented the project at class meetings and a large orientation meeting given at each agency during the first weeks of Head Start. After those meetings, the project director and field staff discussed the project with parents and distributed flyers about the project. Interested parents signed informed consent forms to participate in the project. Every effort was made to contact parents of all of the children. For those parents who missed both meetings, field staff approached them individually as they brought their children to school, gave them a flyer, described the study to them, and had them sign informed consent forms if they were interested in participating in the project. Some mothers took the flyers home after a meeting or individual contact, and agreed to participate a few days later.

Primary caregivers were the subjects of the study because traditionally they have been the targeted parent for most of the parent activities and experiences offered by Head Start programs. In addition, research on family networks among the poor (Allen, 1978; Stack, 1974; Staples, 1971) has demonstrated that the biological mother is frequently not the primary caregiver. A grandmother, aunt, cousin, or foster parent may take on this role. In this study, the biological mother is the subject in most cases; however, we do include the foster parent, grandmother, or aunt when appropriate. For this study the term "mother" is used to denote the primary caregiver of the Head Start child.

One school-aged sibling between the ages of five and twelve was selected by the research team from each research family having a sibling within that age range, so that all of the designated age groups were represented and gender balanced. For Cohort I, 86 siblings were included from the Agency One sample and 36 from the Agency Two sample. 
- Head Start can be either a one-year or two-year program for preschool-aged children (three to four years old). The majority of Head Start children in the study were four-yearolds (Agency One: 132; Agency Two: 57) with no previous Head Start (Agency One: 74\%; Agency Two: 64\%). Including parents, Head Start children, and selected siblings, the full sample of individuals in the longitudinal study is over 1000 .

\section{Data Collection Procedures}

In the fall of 1991, pretest data were collected for Cohort I. Parent data were collected by trained field staff who were of the same ethnic group and/or spoke the same language as the mothers. The Head Start agencies provided private space where field staff administered the measures to small groups of mothers. In most cases the mothers filled out the forms privately and independently, with the field staff available to answer questions when needed. ${ }^{7}$ Confidentiality of the parents' answers were always respected. Completion of the packet of measures by the mothers took up to one-and-one half hours. Mothers received a modest payment for their participation (\$20.00). Some child measures were completed by the teacher, others were completed by the mothers at the same time as the parent measures. Posttest data from Cohort I were collected at the end of the Head Start program year (spring, 1992) using similar procedures.

For the kindergarten follow-up (Cohort I, Agency One -- June, 1993), a telephone interview was conducted with the mother to gather information on her educational, job training and employment activities during the year following Head Start, and on the older sibling's academic and social competence. In a few cases, home visits were made when the family had no telephone. Telephone-interviewed mothers went to the Head Start center to receive their payment for participation $(\$ 20.00)$. Every effort was made to follow up all parents at each data collection point. However, some families left Head Start during the course of the year, some moved out of state or country, and some mothers did not complete the posttest parent measures even though they were still in the Head Start program.

Data were collected from the Head Start child's kindergarten teacher on the mother's involvement in elementary school, and on the child's adaptation to the

\footnotetext{
${ }^{7}$ However, at one agency, several mothers were not able to read the measures on their own. In that case, the field staff took each measure in turn, read the questions one-by-one and the mothers independently filled in the answers.
} 
kindergarten classroom. Teachers also rated the Head Start child's kindergarten performance and anticipated future performance.

\section{Measures}

Described below are the measures employed to assess the major constructs of the study (see Appendix for all measures and Chart A for a list of constructs and sources of data). The measures fall into three major categories. First are measures of the predictor ${ }^{8}$ variables that may predict parent involvement. Second are measures of parent involvement. Third are measures of the outcome variables that may be associated with parent involvement. These data were collected from multiple sources: Head Start parents, children, staff, and from elementary school teachers.

\section{Criteria for Selection of Measures}

Several criteria were used to select the measures for the major constructs and variables of the study:

- Reliability, validity, and norms: Measures with proven reliability and validity were selected where possible.

- Suitability for the study populations: The measures chosen are reliable and valid for low-income populations of various ethnicities including African-American, Latino and White. In addition, age-appropriateness was a major consideration for the Head Start child and older sibling measures.

- Sensitivity to change: Measures that are sensitive to change in individuals, relationships and contexts were selected. Special consideration was given to measures which were sensitive to change that might occur as a result of Head Start experiences and/or parent involvement.

- Appropriateness of reading level and language for parent measures: Measures were chosen that are appropriate to the range of parental literacy levels represented in our study. Measures that had valid Spanish translations were chosen, wherever the other criteria for selection were met. In some cases, where there was no translation or an inadequate translation, or where the measure was created for the study, measures had to be translated into Spanish. The translation process was based on

\footnotetext{
${ }^{8}$ Correlation matrices of the predictor and outcome variables for Agencies One and Two are available from the authors.
} 
the procedure used in previous Head Start research on parent involvement (see below).

- Intrusiveness and time factors: Wherever possible, shorter and less intrusive versions of measures were chosen so as not to undermine the integrity of the research activities.

\section{Translation of Measures into Spanish}

The method used for translation of the measures into Spanish is a common one used in community-based research. The process involved a "blind" reverse procedure using two bilingual people fluent in both written English and Spanish and versed in the terminology of the social sciences. First, one person translated the measure into Spanish from the English version. The second person then translated the Spanish version back into English without looking at the original English version. Then the two English versions were compared and discrepancies in word-meaning were discussed by the two translators and the research staff. Finally, mutual agreement was reached on the most conceptually and linguistically correct translation. In addition, each measure was checked by a group of people from the same ethnic background as the sample (in this case Dominican), for any colloquial discrepancies. These were also corrected by mutual agreement with the research staff.

\section{Measures of Predictors: Demographic, Personal, and Contextual Variables}

Several measures were used to collect pretest information on parents, Head Start children, and their older siblings. From these measures, $41^{9}$ variables were selected and utilized in the analyses examining possible predictors of parent involvement (see Table 1).

The 41 variables included demographic, contextual, and personal characteristics of the families. These variables consisted of characteristics of the mother, the mother's selfsufficiency needs and activities, parenting characteristics, characteristics of the Head Start child and older sibling, and family and community characteristics. The variables were examined to provide a more complete "picture" of the families in the study by identifying

\footnotetext{
${ }^{9}$ For Agency One, there were 40 predictor variables -- all of the variables in Table 1 except ethnicity (parents were 99\% Dominican).
} 
the variables that were potential barriers and facilitators of parents' participation in the Head Start program. The following measures were used to collect the predictor variables:

The Intake/Family Profile was developed by the Head Start Research Group (NCJW Center for the Child, 1992) to collect many of the demographic, contextual, and personal characteristics of the Head Start mother, the Head Start child, and the family from data that already existed as part of the Head Start agencies' records. The majority of the questions came from the Head Start Family Profile that was completed with the parent as part of Head Start's intake procedure. Other data were taken from the child's health record. Variables included: mother's age (variable \#4 on Table 1), Head Start child's age (variable \#24) and gender (variable \#25), number of other children in the home (variable \#38), whether parent had previous experience in Head Start with an older sibling (variable $\# 10$ ), and self-sufficiency needs (variable \#11).

The Norbeck Life Events Scale (Norbeck, 1984) is an 82-item scale which assesses the occurrence of both good and bad life events/experiences in the areas of health, work, school, love, marriage, family and close friends, parenting, personal and social issues, and crime and legal matters. The first part of the scale assesses which events occurred during the prior year. The second part assesses the impact of the event on the parent. Four members of the Head Start Research Group selected the most potentially life-changing events/experiences. These events made up a modified measure of 16 very stressful items, including 14 items that were clearly negative (e.g., death of a loved one, a major illness) and two that could be positive or negative (e.g., beginning or ceasing school, and beginning or ceasing college or a training program). The second part of the Norbeck Scale, the impact of the stressors on the parent, was eliminated because it appeared to be confusing to the parents and, therefore, may have been unreliable. Our modified 16 item composite variable (\#6 in Table 1) yielded one score (range 0 to 16), representing the number of life events/experiences the parent reported for the prior year. A Cronbach's Alpha was not computed for this composite score, since it was not a scale but a variable made up of unrelated items from the different scales in the original measure.

The Maternal Social Support Index (MSSI) (Pascoe, Ialongo, Horn, Reinhart, \& Perradatto, 1988) is a 23-item measure that assesses the degree of social support perceived by mothers. The measure has a total social support scale that includes items on childcare, 
household tasks, contact with extended family and membership in various organizations. The MSSI has been widely used and validated on a range of populations (Pascoe, Chessare, Urich, \& Ialongo, 1987; Pascoe \& French, 1990). Reliability analysis was not appropriate for the Social Support Index because it is composed of different types of items. For this study the total scale was used (variable \#39), since it includes all the social support information described above. The total scale scores could range from 0 to 46 .

Other characteristics of the Head Start Child were measured using the Early Screening Inventory Parent Questionnaire (ESIPQ) (Meisels \& Wiske, 1988). The measure contains questions about the family and about the Head Start child's medical history, health and developmental milestones. For this study, three scales were created covering the following areas: 1) a six item scale about birth-related problems (e.g., low-birth weight) (variable \#26); 2) an eight item scale about health-related problems since birth (e.g., hearing or visual impairment) (variable \#28); and 3) a 35 item scale about age-appropriate developmental milestones (e.g., toilet training) (variable \#27). The scores represent the total number of birth problems, health problems, and developmental milestones not yet achieved, respectively. Due to the relative independence of items in these scales (e.g., there is no reason why vision impairment would be related to hearing impairment), alphas were not computed.

Characteristics of an older sibling such as gender (variable \#31), and age (variable \#30), as well as questions pertaining to his/her medical history and health were assessed by the About Your Older Child Questionnaire (NCJW Center for the Child, 1991), adapted in part from the ESI-PQ by the Head Start Research Group for this study. The questionnaire was completed at pretest only by parents with an older sibling in the study, and included a scale of six birth-related items (variable \#32) and a health-related scale of eight items (variable \#33). The independence of items on the questionnaire rendered it inappropriate for analysis of internal consistency.

Parents were also asked about their perceptions of the safety of their neighborhood (variable \#43). This variable, parents' work, school and job training activities during the Head Start year (variable \#12); pregnancy during the Head Start year (variable \#5), number of years parent is in the U.S. (variable \#1); acculturation (variable \#2), and 
mother's education (variable \#3) were derived from the You and Your Family Questionnaire (NCJW Center for the Child, 1992) administered at posttest.

Maternal depression (variable \#9) was measured by the Center for Epidemiologic Studies Depression Scale (CES-D) (Radloff, 1977). The CES-D is one of the most frequently used measures in the psychological literature to assess depressive symptomatology in normal populations; it can be used to distinguish mild from severe depression in non-clinical samples. Current depressive symptomatology ("how often within the last week") is measured using a 20-item scale, 0 (less than once a week) to 3 (5-7 times a week) for each item. This results in a single item score ranging from 0 to 60 in which high scores indicate more depression, and 16 represents a clinical cut-off point. Parents with scores of 16 and above are categorized as depressed. A Spanish version of the CES-D was available from the Center for Epidemiologic Studies. Internal reliability analysis for our sample on this measure yielded alphas of .86 for English speakers; .87 for Spanish speakers; and .87 overall. This measure was also used to measure depression as an outcome.

Parents' feelings of personal control or mastery in their lives (variable \#8) were assessed by the Pearlin \& Schooler Mastery Scale (MAST) (Pearlin \& Schooler, 1978), a subscale from a larger measure of coping strategies. The mastery subscale consists of seven Likert-type items $(0=$ strongly agree to $4=$ strongly disagree), with total scores ranging from 0 to 28. The subscale assesses subjects' feelings about how much personal control they have in their lives. For this study, a higher score denoted more mastery or personal control. Alphas measuring internal consistency for the measure were .67 for English speakers; .55 for Spanish speakers; and .61 overall. This measure was also used to assess personal control as an outcome.

Self-esteem (variable \#7) was assessed using the Rosenberg Self-Esteem Scale (SE) (Rosenberg, 1965) a 10-item scale measuring feelings of self-worth. Factor analysis of this measure yielded a single global self-esteem factor that accounted for $52.8 \%$ of the variance with all ten items strongly loading on this factor (O'Brien, 1985). Effects of ethnicity and socioeconomic status have been examined and reliability and construct validity have been reported as acceptable (Rauh, Velez, \& Lederman, 1990). For this study, a six-item version was used. Item scores ranged from 4 (strongly disagree) to 1 (strongly agree), giving a total range of 6 to 24, with higher scores denoting lower self-esteem. Internal consistency 
analysis for the six-item version of the measure with our sample produced alphas of .81 for English speakers, .84 for Spanish speakers; and .83 for the total sample combined.

Parent-child relations in the form of attitudes about child-rearing were measured using the Parental Attitudes towards Child Rearing Questionnaire (PACR) (Easterbrooks \& Goldberg, 1984). It contains 51 items, with four subscales: warmth (variable \#15), encouragement of independence (variable \#16), strictness (variable \#17), and aggravation (variable \#18). Scores range from 1 (strongly disagree) to 6 (strongly agree). The strictness subscale measured the harshness of the parent towards the child. Therefore, less strictness indicated a better parent-child relationship. Similarly, less perceived parenting aggravation indicated a better parent-child relationship. Three of the four subscales -warmth, encouragement of independence, and aggravation -- have been significantly related to mothers' scores on a dyadic adjustment scale: Mothers with higher marital adjustment scores expressed warmer attitudes, encouraged toddler independence more and reported less aggravation (Goldberg \& Easterbrooks, 1984; Easterbrooks \& Goldberg, 1984). This measure also has been used previously with low-income African Americans and Latinos (Rauh, Velez, \& Lederman, 1990; Rauh, Wasserman, \& Brunelli, 1990) and a Spanish version was available from that study. For this study, internal consistency analyses yielded alphas from .63 to .68 across subscales for English speakers; from .54 to .80 for Spanish speakers; and from .63 to .77 overall. This measure was also used to assess parenting attitudes as outcomes.

The Parenting Daily Hassles Scale (Crnic \& Greenberg, 1990) is a 20-item measure used to rate two aspects of the stressful day-to-day experiences of parents: the frequency of parenting and childrearing stressful events (variable \#13); and the intensity of parenting stress (variable \#14) experienced by the parent. For this study, a 12-item version of the measure was used, also developed by Crnic and Greenberg (personal communication with M. Greenberg, 1991), which omits an 8-item index of children's problem behaviors. Since behavior problems were being measured separately in this study, the 12 -item version was chosen to reduce redundancy and the length of time required for mothers to complete the instruments.

Items measuring frequency of occurrence ("how often each of these happens to you") consisted of four-point Likert scales from 0 (rarely) to 3 (almost always). Therefore, the 
12-item frequency of daily hassles subscale score could range from 0 to 36 . Items measuring intensity of occurrence ("how much of a 'hassle' you feel") were five-point Likert scales that could range from 0 (no hassle) to 4 (very big hassle). Therefore, the intensity of daily hassles subscale scores could range from 0 to 48 . Factor analyses (Crnic \& Greenberg, 1990) supported the validity of the dimensions of frequency and intensity of parenting daily hassles.

In the present study, reliabilities for the frequency of daily hassles subscale were adequate for both English speakers (.74), Spanish speakers (.71), and for English and Spanish-speakers combined (.74). The intensity subscale reliabilities were similar: .81 for English speakers, .78 for Spanish speakers, and .80 for the two combined. This measure was also used to assess parenting hassles as outcomes.

The home learning environment and parent's involvement in their child's education were assessed by the National Evaluation Information System, Part B (NEIS) (Abt, 1988). The NEIS was developed by the Abt Associates for the national Even Start evaluation. It is a compilation of several scales and individual items from other measures. Part B assesses the home-learning environment in several areas: 1) how frequently the Head Start child helps with household tasks (variable \#19); 2) the number of educationally relevant play materials in the home (toys and arts and crafts supplies suitable for young children) (variable \#20); 3) the number of school-readiness skills the parent has helped child learn (variable \#21); 4) how frequently the parent and child talk about school (variable \#22); and 5) parent's expectations for the child's school performance (variable \#23). Internal reliability alphas for these scales ranged from .66 to .77 for English speakers; from .60 to .77 for Spanish speakers; and from .63 to .77 overall.

Siblings' social competence at pretest was measured by the Competency Scale of the Child Behavior Checklist (CBCL) (Achenbach, 1978). This scale is a comprehensive assessment of the child's adaptation to school, school achievement (parent's report), sibling and peer relations, school problems, grade placement, and special needs. The CBCL has three subscales: activities (five items) (variable \#34), social (six items) (variable \#36), and school (four items) (variable \#35), and a total competency score (variable \#37). Alphas were not computed for these subscales since some items were likert and others were dichotomous and therefore could not be combined. 
Because one of Head Start's major goals is to increase the economic self-sufficiency of poor families, we were interested in whether parents' reports of their self-sufficiency needs at pretest were related to the extent of their involvement in Head Start. We conjectured that parents who reported having these needs might become more involved in Head Start as a vehicle for pursuing their self-sufficiency goals. Although pretest data was not available for the entire sample, data on parents' self-sufficiency needs were available for Head Start parents with children beginning their first year of Head Start. This information was on the Intake/Family Profile. The parents were asked if they needed either employment, or education/job training. Parents were classified as having self-sufficiency needs if they responded "yes" to either one or both.

Based on the results of our pilot study of barriers to parent involvement in Head Start (Parker, Piotrkowski, Peay, Clark, Yoshikawa, Kessler-Sklar, \& Baker, 1996), we anticipated that parents who were engaged in self-sufficiency activities during the Head Start year would have less time to be involved in Head Start. Although pretest data were not available on parents' self-sufficiency promoting activities at pretest, data were available from the About You and Your Family Questionnaire on parents' engagement in these activities during the Head Start year (variable \#12). From this data, parents were classified as "engaged" if they had engaged in at least one of the following three activities: 1) paid employment (part-time or full-time); 2) job-training; or 3) education.

\section{Measures of Parent Involvement in Head Start}

The Parent Involvement Information Protocol (PIIP) (NCJW Center for the Child, 1992) was used to assess all of the parent involvement activities in which parents engaged throughout the Head Start year. The PIIP was filled out by Center for the Child research staff from existing Head Start records originally compiled by Head Start staff as part of the normal reporting requirements of the Head Start program. Each conference, activity, workshop, meeting, home visit, substantive phone conversation, and trip in which the mother participated was recorded by Head Start staff, then sorted by the research group as specific activities and experiences (PIIP, Part A) or as social service utilization (PIIP, Part B). 
Part A of the PIIP was developed to assess the extent of each parent's involvement in specific activities and experiences offered by the Head Start agencies. Because activities differed somewhat at the two agencies, separate forms were developed. For each agency, a list was compiled of names and dates of all of the activities that took place during the Head Start year. Data on parents' participation in each activity or experience were compiled from Head Start attendance sheets and monthly volunteer time sheets.

Part B of the PIIP at Agency One consisted of a list of parents' contacts with Head Start Social Service/Parent Involvement staff for counseling or intervention purposes, over the course of the Head Start year. This information was compiled by research staff from staff notes and other standard forms used by the Head Start staff (e.g., Home Visit Report, Ongoing Social Services notes). Each contact was dated and classified by topic of discussion (e.g., housing, transportation, etc.).

At Agency Two, the Social Services component of the Head Start program functioned differently from Agency One -- and non-traditionally for Head Start. Agency Two is affiliated with a community mental health center, so that its social service needs are managed seamlessly by referral to the professional staff at that center. Consequently - and unfortunately for this study -- the mental health center retained its own records, making them inaccessible to the research team. Hence, the Agency Two PIIP consists only of Part A. No information on social service contacts could be included.

Consistent with our conceptualization and analysis of parent involvement in Head Start, several parent involvement variables were created from the raw PIIP data.

Undifferentiated Involvement was a summary score representing the number of hours spent in all of the activities and experiences listed on the PIIP-A. This included volunteering in the classroom, office, kitchen and on trips; and attending educational workshops, policy committee meetings and other policy-related activities. The number of hours parents utilized social services were not included in the Undifferentiated Involvement variable because the process of parents' utilization of social services were conceptualized as qualitatively different from the processes involved in participating in the other parent involvement activities. Parents who utilized social services_more tended to have more problems than the average Head Start parent. Thus, the pattern of relationships between this type of involvement and outcomes might be different than that of other types of 
involvement and outcomes. Therefore, the relationships between Utilization of Social Services and outcomes were reported separately.

A categorical Undifferentiated Involvement variable was also created to test for non-linear relationships between involvement and the outcomes. The variable was constructed by dividing the full pretest sample of parents at each agency into three groups -- lowest third, middle third, and highest third -- based on total number of hours.

Two parent involvement "activity types" also were created. These variables represented not just amount of involvement but type of activity or experience as well. These classifications were based on, but not identical to, those in the Head Start Performance Standards. As stated earlier, the Head Start Performance Standards identified four groups of activities: Volunteering, Workshops, Policy activities, and Social Services. However, preliminary analyses of our data indicated that attendance at workshops and participation in policy activities should be combined for the following statistical and theoretical reasons: 1) the variability within each of these categories was not sufficient to warrant separate analysis; and 2) these two types of experiences were theoretically linked as "group-oriented" parent experiences versus volunteering which represented an "individual-oriented" parent experience (Herr \& Halpern, 1991).

Volunteering was seen somewhat differently by the two Agencies in our study. At Agency One separate records of attendance on trips were not kept. Instead the data were combined with the volunteer data. This was conceptually sound since parents volunteered their time for trips similarly to how they volunteered their time in the classroom (i.e., served as additional "teachers"). Neither agency kept separate data on volunteering in the classroom, office, and kitchen. In order to be able to compare the results of the two agencies, we created a volunteering variable that included all of these types of volunteering. Therefore, the two parent involvement activity types analyzed in the present study were: 1) Volunteering in the classroom, office, kitchen and on trips; and 2) Attendance at Workshops and Policy Meetings. The third type of activity, parent contact with Social Services, was treated separately as stated above.

In computing scores for these two variables, the Head Start Research Group determined the hour-equivalent for each type of activity, since for the most part, Head Start records did not include this data. Hour-equivalents were based on the number of 
hours usually spent on that activity (information provided by Head Start staff). Each session of volunteering, attendance at workshops and policy-related meetings were three hours long and social service contacts were one hour each. Trips lasted either three or six hours (i.e., half-day or full day, respectively), depending on the kind of trip.

\section{Measure of Engagement in Self-Sufficiency Activities During the Head Start Year}

In the currently difficult socioeconomic climate, one third of the parents in the present study were engaged in self-sufficiency promoting activities outside Head Start (see Results section). In addition, the results of our pilot study of barriers to involvement in Head Start (Parker, Piotrkowski, Peay, Clark, Yoshikawa, Kessler-Sklar, \& Baker, 1996) revealed that engaging in educational and job training activities and having schedule conflicts possibly due to work interfered with becoming involved in Head Start activities, resulting in lower involvement for engaged parents and in non-homogeneity of the parents at different levels of participation. We suspected that parents engaged in self-sufficiency promoting activities during the Head Start year might experience Head Start activities differently and be affected differently by them. Thus, the effect of involvement on an outcome might in part depend on whether parents were also engaged in self-sufficiency activities.

Information about parents' job, job training and educational activities during the Head Start year was collected in the About You and Your Family Questionnaire. Parents were categorized as "engaged" if they were involved in at least one of these activities sometime during the year. Parent who engaged in none of the activities were classified as "not engaged".

\section{Measures of Post Head Start Outcome Variables ${ }^{10}$ - Posttest}

\section{The Parent}

The major outcome for parents in this study was social competence. Indicators of social competence included: adequacy of parenting skills as evidenced by the parent-child relationship; extent to which parents provided a positive home learning environment;

\footnotetext{
${ }^{10}$ Not all predictor variables were included as outcomes in this study.
} 
degree of subjective well-being; and engagement in behaviors that promote socioeconomic self-sufficiency. The measures used to assess these constructs are listed below. Many of the measures were also used to assess demographic, personal, and contextual variables that might predict involvement and, therefore, were described more fully above.

\section{Parenting}

Parent-child relations in the form of attitudes about child-rearing were measured using three subscales of the Parental Attitudes Towards Child Rearing Questionnaire (PACR) (encouragement of independence, aggravation, and strictness subscales) (Easterbrooks \& Goldberg, 1984) and the Parenting Daily Hassles Scale (Crnic \& Greenberg, 1990).

\section{Home Learning Environment}

The home learning environment and parent's involvement in her child's education were assessed by the National Evaluation Information System, Part B (NEIS) (Abt, 1988).

\section{Subjective Well-Being}

Depression was measured by the Center for Epidemiologic Studies Depression Scale (CES-D) (Radloff, 1977). Parents' feeling of mastery or control in their life was assessed by the Pearlin \& Schooler Mastery Scale (MAST) (Pearlin \& Schooler, 1978).

\section{The Head Start Child}

The major outcome for the Head Start child was social competence. Indicators of social competence included: extent of normative social skills and behaviors at home and degree of school readiness.

Social competence in the form of positive normative social skills and behaviors at home were assessed with the Adaptive Social Behavior Inventory (ASBI) (Hogan, Scott \& Bauer, 1991). The $A S B I$ was selected because it has been found to be sensitive to the influences of educational and day care experiences, was appropriate for three and four year olds, and was developed on a diverse population, including African Americans and Latinos (Hogan, Scott \& Bauer, unpublished paper). Two of the subscales -- compliance, and disruptiveness -- were used. The compliance subscale primarily contains items assessing the cooperativeness of the child. The disruptiveness scale contains items assessing the child's demanding as well as assertive behaviors. Theory and normative data collected on the disruptive scale indicated that scores of approximately 11-14 are normal for this age group. Internal reliability analyses yielded alphas of .70 (english speakers) and 
.67 (Spanish speakers) for the compliance subscale; and .66 (English speakers) and .62 (Spanish speakers) for the disruptiveness subscale.

School readiness competencies in the domains of language, cognitive, and motor development were assessed by the Cooperative Pre-School Inventory (CPI) (Caldwell, 1974). This 64-item inventory yields one score that assesses personal-social responsiveness, associative vocabulary, numerical, and sensory skills. The measure has been used successfully with low-income populations in intervention evaluations. The CPI was administered to the child by a Head Start teacher other than her or his own in order to control for possible teacher bias. Internal consistency alphas for this measure were high: .95 for English speakers; .87 for Spanish speakers; and .93 overall.

\section{Measures of Outcome Variables - Kindergarten Follow-up (Agency One)}

The following measures were used in the kindergarten follow-up of Agency One families whose Head Start child was in kindergarten:

\section{The Parent}

The Telephone Interview Protocol (TIP) (Parker, Kessler-Sklar, Peay, Clark, Piotrkowski, \& Baker, 1993) was developed for the follow-up portion of the longitudinal study to assess the status of the family one year after leaving Head Start. Data were collected on the mother, the Head Start child, and the older sibling. The TIP was translated into Spanish, using the blind reverse translation method (see above) and administered by bilingual research assistants. The interview took 30 to 40 minutes. For the mother, the protocol was designed to elicit information on skills and behaviors that promote socio-economic self-sufficiency (not being pregnant, education, job training, and employment). A combined education/job training variable was created to measure the educational component of self-sufficiency.

Parent Involvement Survey - Version T (PIS-T) (for teachers) (Baker, 1993), is a 27item questionnaire completed by the child's kindergarten teacher at the end of the school year (Cohort I) representing the teacher's perception of the parent's involvement in the child's school experience. Although the questionnaire was pilot-tested using focus groups of teachers, the teachers in the present study had sufficient knowledge of parents' involvement on only twelve of the 27 items, including parents' calls, visits, and discussions 
with the teacher; monitoring of homework; help in the classroom and on trips; and whether or not the parents showed respect for school personnel and seemed interested in what was happening in school. In contrast, many of the teachers lacked knowledge about the parent's involvement with the child's extra educational activities at home, and/or about the parent's utilization of school resources such as social services and parenting and selfimprovement workshops. Because of the large amount of missing data for these items, they were eliminated from subsequent analyses.

The 12 types of parent involvement that teachers knew about were factor analyzed. The best solution was a two-factor solution yielding a general 'involvement' factor and a second, orthogonal 'helping' factor which represented parents' participation and/or offers to participate in the classroom. These two factors accounted for $59 \%$ of the variance. Two scales were created based on the factor analyses: (1) an Involvement in Elementary School subscale; and (2) a Helping in Elementary School subscale.

The factor analyses were followed up by reliability analyses of the two subscales. The Cronbach alphas were .87 and .86 , respectively, suggesting that these two scales were viable. The two scales were significantly and moderately correlated with each other $(r=.40, p<.01)$, sharing approximately $16 \%$ of their variance.

\section{The Head Start Child}

The Head Start child's school functioning at the end of the kindergarten year was assessed by the Child's Classroom Adaptation Inventory-Spring (CCAI). This inventory was developed by Halpern and revised by Baker and Piotrkowski (1993) for the Home Instruction Program for Preschool Youngsters (HIPPY) evaluation study (Baker \& Piotrkowski, 1995). The $C C A I$ is a 14-item teacher report that assesses the child's adaptation to the classroom, and motivation and interest in learning. A factor analysis of the data in the present study yielded two factors: 1) adaptation to the classroom; and 2) teacher ratings of child's current performance and expectations for future performance. The reliability of the two scales were: .96 and .94 , respectively. The scales were highly intercorrelated $(r=.87)$, but were conceptually distinct and were analyzed separately.

\section{The Older Sibling}

The major outcome for the older sibling (ages 7 through 14 at time of follow-up) of the Head Start child was social competence. Indicators of social competence included: 
positive relationships with family and peers, appropriate academic performance, being on grade level, and not being placed in a special education class. Siblings' social competence was measured by the competency scale of the Child Behavior Checklist ( $C B C L$ ) (Achenbach, 1978) completed by the parent. This scale assesses three types of social competence: 1) activities (five items) which assesses level of participation and skill in sports and hobbies; 2) social (six items) which assess sibling, family and peer relationships, and participation in structured organizations and 3) school (four items) which assess grades, special placement, retention in grade, and school problems. There is also a total competence score which is the mean of the three competence scales. At follow-up, the $C B C L$ competency scales were included in the Telephone Interview Protocol (TIP).

\section{Overview of Data Analyses}

The data analyses addressed Objective 1 -- the predictors of involvement -- and Objective 2 -- the outcomes associated with involvement. In reporting our results, we considered both statistical significance level and effect size. In testing for statistical significance, ${ }^{11}$ more conservative two-tailed tests were used to minimize Type I errors and to increase the interpretability of our findings. In considering effect size, we used Cohen's (1977) definitions of small, medium, and large effect sizes as correlations of .10, .30, and .50, respectively. All of these effect sizes are considered to be meaningful (Cohen, 1977). However, with the sample sizes available in each Agency, the present study had a power of only $50 \%$ to detect meaningful effects. In order to increase our power to an acceptable level of .75 , we report findings with $p$ values less than .10 . $P$ values less than .05 were reported as statistically significant and $\mathrm{p}$ values between .05 and .10 were reported as tending towards statistical significance.

Because this study examined the effects of parent involvement on many outcomes, it was necessary to perform many statistical tests. Therefore, we identified those instances where the number of statistically significant findings and trends in relation to the total number of tests conducted was below chance ( $10 \%$ of tests conducted). When the number

\footnotetext{
${ }^{11}$ When used in the Results section, the term "significance" will refer to statistical significance.
} 
of statistically significant tests and trends was greater than ten percent, it was concluded that the results were probably not due to chance.

\section{Description of the Sample}

The demographic, contextual, and personal characteristics of the families at pretest were described. This provided a context within which to interpret our findings. Parents', children's, and siblings' pretest and posttest scores on outcome measures are reported in the sections pertaining to those outcomes.

\section{Analysis of Parent Involvement Data}

Because the parent involvement variables were not normally distributed, they were transformed so that parametric statistical techniques could be employed. Logarithmic and square root transformations were performed, resulting in normally distributed variables.

\section{Changes in Sample Size over the Course of the Study}

Attrition analyses were conducted to identify the populations of families to whom our results could be generalized. Attrition rates were computed for the posttest, telephone interview and kindergarten samples. The groups then were compared with respect to the variables listed in Table $3 .^{12}$

The following groups were compared: (1) those who completed the pretest and posttest versus those who completed only the pretest; (2) Those who were in the kindergarten telephone interview follow-up $(N=115)$ versus those who completed the pretest but were not included in the follow-up; and (3) Those who were in the kindergarten teacher data follow-up $(\mathrm{N}=119)$ versus those who completed the pretest but were not included in the follow-up. There were several reasons why some families were not included in one or the other kindergarten follow-up. These reasons were: (a) they did not complete the posttest; (b) they completed the posttest but were not eligible for the

\footnotetext{
12 Data on the following variables were not available for non-posttested families: mother's country of birth, number of years in the U.S., acculturation, highest grade mother completed, whether pregnant during the Head Start year, and number of years child was in Head Start. Therefore, the families could not be compared on these variables.
} 
kindergarten follow-up because the Head Start child was too young to go to kindergarten;

(c) they were eligible for the follow-up but either refused or could not be found.

\section{Predictors of Parent Involvement}

For those parents who completed the posttest, a series of analyses was performed to identify predictors of the parent's involvement during the Head Start year. Zero-order correlations were computed between the demographic, contextual, and personal characteristics of the families at pretest (see Table $1^{13}$ ) and the four parent involvement variables. These analyses yielded groups of variables that were associated with each type of involvement.

To learn more about the predictors of each type of involvement, we then conducted stepwise multiple regression analyses. These analyses addressed two related questions: (a) What was the total percentage of variability in involvement predicted by the variables; and (b) What was the unique contribution of each variable in predicting each type of involvement.

\section{Association of Parent Involvement with Parent, Child and Sibling Outcomes}

At Agency One, outcome data were collected both at the end of the Head Start year and again one year later. Thus, for Agency One, outcome data were available on five parent outcome constructs -- at the end of the Head Start year: the parent-child relationship, the home learning environment, and parents' psychological well-being; and during the follow-up year: engagement in self-sufficiency promoting activities during the year; and involvement in the Head Start child's elementary school. For Agency Two, outcome data were collected only at the end of the Head Start year and, thus, data were available on the three outcome constructs assessed at that time.

Similarly, for Agency One, Head Start child outcome data were available both at the end of the Head Start year and at the end of the kindergarten year. Head Start older sibling outcome data were available only for Agency One and only at the end of the

\footnotetext{
13 The predictors of Agency One involvement include all variables in Table 1 except ethnicity because $99 \%$. of the Agency One sample was Dominican. The predictors of Agency Two involvement include all variables in Table 1.
} 
kindergarten year. For Agency Two, Head Start child outcome data were available only at the end of the Head Start year.

In order to provide a comprehensive test of our hypothesis, we chose our outcomes to represent distinct, non-overlapping, orthogonal categories. Within each category, we also chose our outcome variables to be distinct. Nevertheless, we considered the possibility of using data reduction techniques to combine variables within constructs and/or to combine constructs. However, factor analyses performed on the Agency One outcome data did not yield a clear set of meaningful outcome factors. We also attempted to combine scores for variables which theoretically were related. We found that in many cases, the shapes of the distributions of the outcome variables differed too much to make it meaningful to do so. Other sets of variables were subscales of individual measures constructed to be distinct and were, therefore, not amenable to combination (e.g., aggravation and strictness scales of the PACR).

In order to test our hypothesis, first, partial correlations were computed between the involvement measures and the outcomes, controlling for initial pretest scores on those outcomes. For those outcomes which, as predicted, were significantly and positively related to involvement or showed a trend towards statistical significance, ${ }^{14}$ we then addressed the following question: Would the relationship between involvement and the outcome be maintained after controlling for important demographic factors which also were associated with the outcome? This constituted a conservative test of the hypothesis. A two-step strategy was employed to answer this question. First, zero-order correlations were computed between nine potentially relevant demographic variables and the outcome. The demographic variables tested for use in the regressions were: ethnicity, ${ }^{15}$ mother's age and educational level, number of years the mother participated in Head Start, number of years the child was in Head Start, number of other children in the home, child's age, child's gender, and child's school readiness as measured by the Cooperative Preschool Inventory.

\footnotetext{
${ }^{14}$ In those cases in which the partial correlation between involvement and the outcome was zero or negative, we conducted additional analyses to explore other, possible complex relationships.

${ }^{15}$ Parents in Agency One were almost all Dominican so ethnicity was used in Agency Two analyses only.
} 
Those variables which were statistically significantly correlated with the outcome or which tended towards statistical significance then were used as controls in a hierarchical regression analysis. In each regression, the order of entry of variables was: 1) demographic variables; 2) pretest scores; and 3) involvement. ${ }^{16}$ The same analytic procedures were followed in testing the hypotheses related to the dichotomous outcomes, with the exception of the substitution of logistic multiple regression. Because in our data set, "yes" in response to the question, "were you pregnant?", or "did you have a paid job?" was coded "1", and "no" was coded "2", the analyses actually predicted the probability or odds of not having attended school or participated in job training, not having been pregnant, and not having had a job during the year after Head Start.

An additional set of analyses explored the relationships between the two major types of involvement activities (Volunteering, Workshops/Policy Meetings) and the outcomes. The same partial correlational and regression procedures were used in these analyses as in the analyses involving Undifferentiated Involvement. Additionally, if both activity types showed statistically significant relationships with the outcome, two additional hierarchical multiple regressions were performed that controlled for the pretest and varied order of entry of the two activity types. These analyses addressed the question of whether either activity type had an impact on the outcome over and above that of the other.

\section{Exploration of Complex Relationships Between Involvement and Outcomes}

When either no linear relationship or a negative relationship was found between the three involvement variables and an outcome, several additional analyses were performed to explore alternative complex relationships. To permit comparison with previous studies, these analyses were conducted with Undifferentiated Involvement as the independent variable.

The first analysis tested for a non-linear relationship between involvement and the outcome, with Undifferentiated Involvement measured categorically. This was done with a oneway analysis of covariance on the outcome.

\footnotetext{
${ }^{16}$ The overall models were always significant because of the inclusion of pretest scores, which are excellent predictors of the outcomes.
} 
A second analysis tested for a linear relationship between Undifferentiated Involvement and the outcome after controlling for all of the pretest or predictor variables in the study (see Table 1) that were statistically significantly correlated with the outcome. Controlling for these correlates increased the precision of the analysis by adjusting the outcome scores for a wide range of variables on which parents differed, eliminating extraneous variability. To perform this analysis, first zero-order correlations were computed between each predictor variable and the outcome. Those that were statistically significant or tended towards statistical significance were entered in Block 1 of a hierarchical regression analysis, followed by pretest scores in Block 2 and the continuous Undifferentiated Involvement variable in Block 3.

The last analysis examined the interaction between involvement and engagement in self-sufficiency promoting activities during the Head Start year. The categorical Undifferentiated Involvement variable and a dichotomous engagement variable (engaged, not engaged) were used, creating six groups: 1) low involved not engaged; 2) medium involved not engaged; 3) high involved not engaged; 4) low involved engaged; 5) medium involved engaged; and 6) high involved engaged. The questions we addressed were: 1) Is there a difference between the six groups at pretest? 2) Is there a difference between the six groups at posttest? and 3) What is the pattern of changes from pretest to posttest within the six groups? Several complementary analyses were conducted to answer these questions: 1) An analysis of variance and multiple pairwise comparisons of pretest and on posttest scores for the six groups; 2) Analysis of covariance on the outcome controlling for the pretest; 3) Repeated measures analysis of variance on pretest and posttest outcome scores; and 4) simple effects tests of the magnitude of change in the outcome from prëtest to posttest for each of the six groups. As part of these analyses, the direct effect of engagement in self-sufficiency promoting behaviors on the outcome also was tested. The tests of this effect were similar to the analyses described above, except that there were only two groups -- engaged and not engaged. This third set of analyses was conducted for all of the outcomes with statistically nonsignificant partial correlations when sample size allowed. 


\section{RESULTS: AGENCY ONE}

\section{Demographic, Contextual, and Personal Characteristics of the Sample at Pretest}

The families that participated in the study at Agency One $(\mathrm{N}=203)^{17}$ tended to be homogeneous in terms of culture (Dominican), and language (Spanish), but were diverse in other ways. Described below are demographic and contextual highlights of the specific characteristics that participating mothers, children, and families brought to the Head Start experience (see Table 2).

\section{Mothers}

In general, mothers in the Agency One sample were impoverished, poorly educated, and unskilled women, $98 \%$ of whom were Hispanic, with only $1 \%$ each of Euro-Americans and African Americans. Of the Hispanics, most immigrated to this country from the Dominican Republic. Although their years in the United States ranged from 2 through 33, 83\% were born outside the U.S., and $63 \%$ received their formal education in their native country and spoke little English. In $82 \%$ of their homes, Spanish was the dominant language spoken.

Educational levels for the sample were low by mainstream American standards: 43\% did not finish High School (whether they dropped out or their education was interrupted by emigration was unclear); another third of the mothers completed High School or its equivalent; and another quarter received training beyond High School level. Approximately one third of the total sample reported needing additional education, job training, or a job in order to proceed toward economic independence.

With respect to age, Agency One mothers were relatively mature: they ranged from 21 to 62 years, with most being between 27 to 40 years of age, and the average age being 34 years. Only $5 \%$ gave birth to their Head Start child as adolescents.

\footnotetext{
${ }^{17}$ One hundred seventy-two of these families completed the posttest.
} 


\section{Head Start Child and Older Sibling}

For nearly three-quarters of the 203 Head Start children in the sample, Head Start was a new experience. The other quarter of the children were beginning their second year. The children ( $51 \%$ girls) ranged in age from two years ten months to four-and-a-half years of age, with nearly two thirds being four-year-olds. Eighty-six older siblings also participated in the study, $56 \%$ of whom were girls. They ranged in age from five to 12 years, with the average being just under eight years of age ( 7.7 years).

\section{Families}

The majority of families at Agency One tended to be relatively small and were headed by single women. Specifically, mothers were most often the only adult residing in their home -- only $33 \%$ lived with one or more other adults (a spouse or a parent, for instance) -- at the beginning of the Head Start year. Most often, mothers reported nuclear families of one child in addition to the Head Start child (50\%); a fourth reported two other children; only $10 \%$ of mothers reported families of 3 or more other children; and $12 \%$ of the mothers reported a pregnancy or another birth during the Head Start year. In slightly over half of the families, the Head Start child was the youngest. Thirty-eight percent of mothers reported one younger child, and for only $6 \%$ of the families were there two or more other children younger than school age.

Data on material resources available to this group of Head Start families reflected considerable poverty. For $82 \%$, the annual income was $\$ 10,000$ or less. Of the $77 \%$ of mothers who answered the question on receipt of Aid to Families with Dependent Children, 99\% reported that some or all of the family income was derived from this source (AFDC). Additionally, 59\% reported problems with their living space. That is, they lacked dependable utilities and/or their space was too small or needed repairs.

\section{The Neighborhood}

In a community where 50 murders, 21 rapes, 478 assaults, and 772 robberies occurred in 1992 per 100,000 residents (NYPD Annual Statistical Report, Garfield \& Abramson, 1994), Agency One mothers agreed: their assessment of the neighborhood was 
that it was indeed unsafe. Nevertheless, $90 \%$ reported that they intended to remain there the following year.

\section{Changes in Sample Size Over the Course of the Study}

\section{Changes from Pretest to Posttest}

Two hundred and fifty-six families were eligible to be included in the study, and 203 of these families (79\%) agreed to participate. Of the 203 families, 172 , or $84 \%$, also completed the posttest. This represented an attrition rate of $16 \%$. All but one family who did not participate in the posttest moved away from the area. These families "disappeared" and were unavailable for posttesting. It was suspected by the Head Start agency that they moved back to the Dominican Republic.

In order to determine the population of families to whom our results would generalize, a series of analyses were performed comparing the families who were posttested $(\mathrm{N}=172)$ to the families who were not $(\mathrm{N}=31)$. T-tests were computed comparing the two groups on 24 demographic, contextual, and personal variables of interest to the study, measured at pretest (see Table $3^{18}$ ). Of the 24 t-tests computed, only two were statistically significant, less than the number expected by chance. ${ }^{19}$ Thus, pretest to posttest attrition did not appear to be a factor affecting the generalizability of the data collected at the end of the Head Start year.

\section{Changes from Pretest to Kindergarten Follow-up: Telephone Interview Sample}

At Agency One, a follow-up assessment of the parent and sibling was conducted one year after the completion of the project with those families whose.Head Start child had just completed kindergarten. Data on parents' self-sufficiency-promoting activities and siblings' social competence were collected from parents by telephone interview. One hundred fortyfour families were eligible for this follow-up. Of these we were able to contact 115 families by phone. This represented an attrition rate of approximately $20 \%$.

\footnotetext{
18 The eight sibling variables in Table 3 were not included in the comparisons of the posttested and non-posttested families because only 9 of the 85 families with older siblings were not posttested. This did not constitute a large enough group to compare to the 76 families with older siblings who were posttested.

${ }^{19}$ Mothers who were posttested reported feeling significantly less personal control in their lives $[t(191)=2.03, p<.05]$, and allowed their children to help with household tasks more often $[t(185)=-2.11, p<.05]$.
} 
We compared the families who were followed-up by telephone $(N=115)$ to the families who completed the pretest but who were not followed-up at kindergarten. This group of families $(\mathrm{N}=88)$ included 31 families who completed the pretest but did not complete the posttest, 28 families who completed the posttest but who were not eligible for the kindergarten follow-up, and 29 families who completed the posttest and who were eligible for the kindergarten follow-up, but who could not be found.

The two groups were compared on 32 demographic, contextual, and personal variables measured at pretest. ${ }^{20}$ As expected, the two groups differed in age of Head Start child because age constituted the selection criterion for follow-up. The two groups differed in three additional ways, representing less than $10 \%$ of the tests performed, the level expected by chance. ${ }^{21}$ Thus, attrition did not appear to affect the generalizability of the data collected in the telephone interviews.

\section{Changes from Pretest to Kindergarten Follow-up: Teacher Data}

The follow-up assessment conducted one year after the completion of the project with those families whose Head Start child had just completed kindergarten also included teacher assessments. Teachers rated the classroom adaptation of the Head Start child and the involvement of the parent in public school. The sample of families rated by the teachers was slightly different from the sample who were contacted by telephone. In the telephone interviews, parents were asked which school their child was attending. However, twelve of these families were not known to their specified school and could not be found in neighboring schools. School data also were collected on 16 families who were eligible for follow-up but who could not be reached for the telephone interview. Thus, 119 children of the 203 who were pretested were followed up in public school. The 84 families for whom school data were not available included those who never completed the posttest $(\mathrm{N}=31)$, those not eligible for:follow-up $(\mathrm{N}=28)$, and those who were eligible but for whom the school could not be identified $(\mathrm{N}=25)$.

\footnotetext{
${ }^{20}$ The sample size of the group of families with siblings was large enough to allow comparisons of siblings' pretest characteristics for these two groups.

${ }^{21}$ The $t$-tests indicated that follow-up mothers: 1 ) were significantly older than non-follow-up mothers [t(195) $=-2.11$, $\mathrm{p}<.05] ; 2)$ reported feelings of personal control/mastery that were significantly lower $[t(191)=2.43, p<.05]$; and 3$)$ reported a significantly greater intensity of daily parenting hassles $[t(168)=-2.01, p<.05]$.
} 
The two groups were compared on 32 demographic, contextual, and personal variables measured at pretest. ${ }^{22}$ As expected, the two groups differed in age of Head Start child because child's age constituted the selection criterion for follow-up. With respect to the remaining 31 variables, the groups differed in three ways, at chance level. ${ }^{23}$ Therefore, attrition did not seem to be a factor affecting the generalizability of the school follow-up data.

\section{Siblings Followed at Kindergarten Follow-up Versus Siblings Not Followed}

Outcome data also were collected for those older siblings of families included in the telephone survey. Of the 86 families at Agency One whose Head Start child had an older sibling enrolled in the study and for whom pretest data were available, 47 were followed $(55 \%)$. This represented a large rate of attrition.

In order to determine if the generalizability of the sibling data was affected by the attrition, the two groups of families (followed versus not followed) were compared on the same 32 demographic, contextual, and personal variables. They differed in three ways, or on $9 \%$ of the tests performed, below the $10 \%$ chance level. ${ }^{24}$ The two groups did not differ on the major pretest variables relevant to the interpretation of the results of the study.

\section{Description of Parents' Involvement in Head Start}

We began by examining the frequency distributions of parent participation in Head Start in order to understand how much and in what ways the parents involved themselves. While virtually all of the parents participated in some Head Start parent involvement activities (202 out of 203 , or $99.5 \%$ ), there was considerable variation from parent to parent (see Table 4):

\footnotetext{
${ }^{22}$.The sample size of the group of families with siblings was large enough to allow comparisons of siblings' pretest characteristics for these two groups.

${ }^{23}$ The follow-up group had older mothers and older "older" siblings. The two groups also differed with respect to one of the sibling variables: The Follow-up group tended to have greater overall social competence $[t(68)=-1.83, p<.10]$ as indicated by the CBCL.

${ }^{24}$ Follow-up mothers were more depressed at the beginning of the study $[t(84)=-2.09, p<.05]$; and they reported feelings of less personal control/mastery $[t(84)=2.85, p<.01]$. Follow-up mothers also reported more health-related problems for their child prior to Head Start $[t(79)=-2.43, p<.05]$.
} 


\section{Undifferentiated Involvement}

This variable represented the number of hours parents spent volunteering and attending workshops and policy meetings. Nearly all parents participated in one or more of these activities (201 out of 203, or $99 \%$ ), but individual involvement varied widely (from 0 to 342 hours). The average number of hours was 90 (nine hours per month), and the median was approximately 80 hours (eight hours per month). The non-normal distribution of this variable was corrected by a square root transformation.

The data on Undifferentiated Involvement was compared with that of Parker, Piotrkowski and Peay (1987), an earlier study at the same agency. Parents ten years ago spent approximately twice as many hours in activities as the parents in the current study such that the high participators in the present study would have been categorized as low participators in the earlier study. Because of the large difference in overall level of participation between the two studies, the ranges of participation defining "low", "medium" and "high" involvement were not equivalent. Therefore, the findings of the present study will need to be understood in light of these differences and will be discussed in that context in the Discussion section.

We conjectured that in the present harsher economic climate, more of the present study's parents were engaged in essential activities outside Head Start (work, going to school, job training) than in the earlier study. In fact, in the earlier study, approximately one-fifth of the parents were engaged in some sort of self-sufficiency promoting activity during the Head Start year. In the present study, approximately one-third were so engaged. Moreover, these engaged parents participated statistically significantly.less in Head Start parent involvement activities than non-engaged parents (see Predictors of Involvement portion of Results section).

\section{Involvement Differentiated by Type of Activity}

The two types of activities included in Undifferentiated Involvement were: (a) Volunteering; and (b) Workshops/Policy Meetings. Parents volunteered an average of 52.9 hours over the course of the Head Start year. The median number of hours volunteered was 51.0 and participation ranged from zero to 222 hours. Eight-nine percent of the 
parents did some kind of volunteering. A square root transformation was applied to the Volunteering scores to normalize the distribution.

The mean number of hours spent in workshops and policy meetings was 37.0 , the median was 30 hours, and the range was from zero to 150 . Nearly all parents (97.5\%) participated in at least one workshop or policy meeting during the Head Start year. A square root transformation was applied to this data as well in order to normalize the distribution.

\section{Intercorrelations of the (untransformed) Parent Involvement Variables}

As expected, Volunteering and Workshops/Policy Meetings were highly correlated with Undifferentiated Involvement ( $r=.91$ and $r=.86$, respectively) (See Table 5). The present study will explore empirically whether the two activity types and Undifferentiated Involvement are, nonetheless, conceptually distinct, resulting in different relationships with the parent, Head Start child and older sibling outcomes. The three involvement variables were not significantly correlated with Social Services Utilization ( $r$ 's $=.10$ (Volunteering), .07 (Workshops/Policy Meetings), and .10 (Undifferentiated Involvement), all p's >.10).

Volunteering and Workshops/Policy Meetings were statistically significantly correlated $(\mathrm{r}=.58)$ with each other, but neither was correlated with Utilization of Social Services ( $r$ 's $=.10$ and .07 , respectively, p's $>.10$ ), supporting the hypothesis that Social Services is qualitatively different from the other activity types. Therefore, the data analyses regarding parents' Utilization of Social Services are reported in a separate section.

\section{Summary and Discussion of Parents' Involvement}

In summary, in this sample virtually all mothers participated to some extent. As with the demographic data, it seems that there is not just one "prototypical" Head Start mother. The majority of parents, however, did not spend a lot of time in parent involvement activities.

It is important to note that parents differed in the relative amounts of time spent in the two activity types. Some parents volunteered more and attended less workshops, whereas others attended more policy meetings but volunteered less. Thus, parents' Undifferentiated Involvement was not homogeneous. 


\section{Predictors of Parent Involvement}

\section{- What are the demographic, contextual, and personal variables related to parent participation in Head Start?}

Objective 1, to identify through a longitudinal study, the "predictor" variables associated with parents' participation in Head Start, was addressed in analyses conducted on the families who completed the posttest $(n=172)$. First, correlations were computed between each measure of involvement and $38^{25}$ demographic, contextual, and personal variables relating to characteristics of the mother, Head Start child, and older sibling at pretest (see Table 1).

Next, those predictors which were statistically significantly related to or tended towards a significant relationship with each type of involvement were further examined in stepwise multiple regression analyses with involvement as the outcome (see Table 6). These analyses addressed two questions: 1) What is the total amount of variability in involvement accounted for by those predictors; and 2) Which variables maintained their associations with involvement in the presence of the other "predictors". ${ }^{26}$ Because data on sibling predictor variables were not available for those families without older siblings in the study (57\% of the families who completed the posttest), the sibling variables were not included in the stepwise multiple regression analyses. The results are reported below.

\section{Predictors of Undifferentiated Involvement}

Six of the $38^{27}$ predictor variables were associated with Undifferentiated

Involvement; two were statistically significant and four tended towards statistical significance. Not being pregnant $(r=-.27)$ and experiencing less frequent daily parenting

\footnotetext{
25 This includes all variables in Table 1 except ethnicity, engagement in self-sufficiency promoting activities and parent's need for greater self-sufficiency reported at pretest. Ethnicity was excluded because $99 \%$ of the Agency One sample was Dominican. The other two variables were analyzed separately because of their theoretical importance.

26 The regression analyses were examined for multicollinearity due to the intercorrelations of the predictors. The tolerances indicated that multicollinearity was not a problem in any of the regressions.

27 This includes all variables in Table 1 except ethnicity, engagement in self-sufficiency promoting activities, and parent's need for greater self-sufficiency reported at pretest. Because parents' engagement in self-sufficiency promoting activities occurred over the Head Start year, this variable was not a predictor of involvement, but rather, a correlate. Ethnicity was excluded because $99 \%$ of the Agency One sample was Dominican. Data on parent's need for self-sufficiency was available only for the portion of the sample whose Head Start child was beginning his or her first year in Head Start.
} 
hassles $(r=-.16)$ were significantly associated with more Undifferentiated Involvement. The mother's perceiving less intense parenting hassles $(r=-.16)$, having a child beginning the first year of Head Start as opposed to the second $(r=-.13)$, having a younger Head Start child $(\mathrm{r}=-.14)$, and having a sibling with a higher CBCL school competence score $(r=.21)$, tended to be associated with greater Undifferentiated Involvement in Head Start.

In order to determine the amount of variability in Undifferentiated Involvement accounted for jointly by these variables, as well as the unique contribution of each variable, a stepwise multiple regression analysis was conducted with five of the six variables entered into the equation (see Table 6 ). ${ }^{28}$ The overall regression model was statistically significant (final Multiple $\mathrm{R}=.36, \mathrm{~F}=4.20, \mathrm{p}<.001$ ) and the percent of variability accounted for jointly by the five predictor variables was $9.7 \%$. In the presence of all five predictors, not being pregnant and experiencing less frequent parenting hassles each accounted for statistically significant variability in parents' Undifferentiated Involvement. Child's age tended towards statistical significance. Neither number of years the child was in Head Start nor the intensity of parenting hassles were independent predictors in the presence of the other three variables.

This pattern of relationships is not surprising. Child's age and number of years the child is in Head Start are somewhat redundant -- younger children tended to be in their first year of Head Start rather than in their second year $(r=.44, p<.0001) .{ }^{29} 1$ Similarly, because frequency and intensity of daily hassles were highly correlated $(\mathrm{r}=.66, \mathrm{p}<.0001)$, the information provided by intensity of hassles was redundant with that provided by frequency of hassles. ${ }^{29}$ The total percentage of variance jointly accounted for by the predictors was statistically significant, but low. Therefore, although the range of possible predictors tested in this analysis was large, it is evident that there are other influences affecting extent of parents' Undifferentiated Involvement that remain to be identified.

\footnotetext{
${ }^{28}$ Data on the sixth variable, sibling's CBCL school competence score, were not available for 98 families who did not have an older sibling in the study, and thus, was omitted from the stepwise regression.

${ }^{29}$ Although these variables are correlated, their tolerances in the regression analysis indicated that multicollinearity was not a problem and that the analyses were valid. 


\section{Predictors of the Activity Types}

\section{Predictors of Volunteering}

Five of the $38^{30}$ predictor variables were associated with Volunteering, of which two were statistically significant: 1$)$ pregnancy $(r=-.24)$; and 2$)$ frequency of daily parenting hassles $(r=-.15)$. Three additional variables tended towards statistical significance: 1$)$ intensity of daily parenting hassles $(\mathrm{r}=-.16)$; 2 ) child's health related problems since birth $(r=.13)$; and 3) child's birth difficulties $(r=-.15)$. Thus at pretest, the parents perceiving fewer and less intense daily hassles, not being pregnant, having a Head Start child with more health problems but with fewer birth difficulties were associated with more hours spent volunteering in Head Start. Four of these five relationships may be explained by the greater amount of time they allowed the parent to spend volunteering. Although having a Head Start child with more birth difficulties was associated with spending less time volunteering, having. a Head Start child with more health problems since birth was associated with more volunteering in the classroom. This may have been due to parents' desire to be with a sick child at the Head Start Center and to their reluctance to leave a sick child in the hands of others. In contrast, a child with more birth difficulties may have been more likely to need home care, or to need frequent visits to the doctor, resulting in the parent's having less time to spend at the Head Start center.

In order to determine the total amount of variability accounted for by the five variables, they were entered into a stepwise multiple regression analysis (see Table 6). The overall regression model was statistically significant (final Multiple $R=.38, F=4.66, p<.001$ ), and the percent of variability in Volunteering accounted for jointly by the five variables was 11.1\%. Four of the five variables were independent predictors of more volunteering when in the equation together: not being pregnant, less frequent daily parenting hassles, less birth difficulties experienced by the Head Start child, and the child's having poorer health since

\footnotetext{
${ }^{30}$ This includes all variables in Table 1 except ethnicity, engagement in self-sufficiency promoting activities and parent's need for greater self-sufficiency reported at pretest. Ethnicity was excluded because $99 \%$ of the Agency One sample was Dominican. Self-sufficiency needs was analyzed separately because data were available only for the subset of Head Start parents whose child was beginning his or her first year in Head Start. Parents' engagement in self-sufficiency promoting activities during the Head Start year was analyzed separately because of its importance as a variable which might potentially moderate the effects of involvement on outcomes.
} 
birth. With the other variables in the equation, intensity of hassles no longer predicted amount of Volunteering. Again, as with the predictors of Undifferentiated Involvement, this was not surprising because frequency and intensity of daily hassles were highly correlated. ${ }^{31}$

\section{Predictors of Participation in Workshops/Policy Meetings}

Two of the $38^{32}$ predictor variables were statistically significantly related to the number of hours the mother spent in workshops and policy-related meetings at the Head Start agency, and a third variable tended towards a statistically significant association, less than the number expected by chance. Because the number of statistically significant predictors was less than that expected by chance, a stepwise regression analysis was not conducted. ${ }^{33}$

\section{Self-Sufficiency Needs at Pretest and Engagement in Self-Sufficiency Promoting Activities During the Head Start Year as correlates of Parents' Involvement}

Two other variables which might affect the extent of parents' involvement were examined. First, we examined the extent to which parents' reported self-sufficiency needs at pretest predicted their involvement. ${ }^{34}$ Second, we explored whether their pursuit of selfsufficiency activities outside Head Start over the course of the Head Start year covaried with extent of involvement in Head Start.

\footnotetext{
${ }^{31}$. Although these variables are correlated, their tolerances in the regression analysis indicated that multicollinearity was not a problem and that the analyses were valid.

${ }^{32}$ This includes all variables in Table 1 except ethnicity, engagement in self-sufficiency promoting activities and parent's need for greater self-sufficiency reported at pretest. Ethnicity was excluded because $99 \%$ of the Agency One sample was Dominican. The other two variables were analyzed separately because of their theoretical importance.

${ }^{33}$ The three variables correlated with attendance at Workshops/Policy Meetings in this sample of Agency One mothers were: 1) not being pregnant ( $r=.22)$; 2) having more social support $(r=.16)$; and 3$)$ the parent's talking less with her Head Start child about school activities $(r=-.13)$. Although some or all of these correlations may represent unreliable associations, it is likely that the relationship between pregnancy and attendance at workshops and policy meetings represents a reliable relationship because the same association of pregnancy with involvement was found with both Undifferentiated Involvement and Volunteering at Agency One, as well as with the three involvement variables at Agency Two.

${ }^{34}$ Data were not available for the subsample of parents whose Head Start child was beginning his or her second year in Head Start.
} 


\section{Self-Sufficiency Needs as a Predictor of Extent of Involvement}

Parents who reported having self-sufficiency needs spent statistically significantly more hours in each of the three types of involvement. Correlations with the three types of involvement were: 1) Undifferentiated Involvement, $r=.27, p<.003 ; 2)$ Volunteering, $r=.20$, $\mathrm{p}<.05$; and 3) Workshops/Policy Meetings, $r=.23, p<.02$. Thus, in this subsample, selfsufficiency needs accounted for up to approximately five percent of the variability in extent of involvement. These results are consistent with the belief that parents become involved in Head Start because they perceive it as a means of having their needs met.

Engagement in Self-Sufficiency Promoting Activities During the Head Start Year and Extent of Involvement

Parents engaged in at least one of three self-sufficiency promoting activities (employment, education, job training) spent statistically significantly less hours in all three types of involvement. They volunteered less $[\mathrm{t}(167)=2.06, \mathrm{p}<.05]$, spent less hours in Workshops and Policy Meetings $[\mathrm{t}(167)=2.69, \mathrm{p}<.01]$, and participated less overall (greater Undifferentiated Involvement $)[t(167)=2.71, p<.01]$ than not engaged parents. The percent of variability in each type of involvement accounted for by this variable ranged between two and four percent (r's ranged from -.16 to -.21.) This pattern is consistent with our belief that such activities conflict with Head Start involvement.

\section{Summary and Discussion of the Findings on the Predictors of Involvement}

The correlations between the "predictor" variables and the measures of involvement were low to moderate in magnitude, with the signs of the coefficients generally in the expected"direction. Together, the predictors, self-sufficiency needs, and engagement in selfsufficiency activities during the Head Start year accounted for $10-20 \%$ of the variability in parents' involvement.

One variable, whether or not the mother was pregnant, predicted all types of participation. Those mothers who were pregnant participated less in Head Start. Related results were found in our pilot study of barriers to involvement in Head Start (Parker, Piotrkowski, Peay, Clark, Yoshikawa, Kessler-Sklar, \& Baker, 1996). In that study, mothers who had a baby or toddler at home participated less as judged by staff. 
Several other variables were related to one or two of the types of involvement. More perceived daily parenting hassles was linked with less Volunteering and less Undifferentiated Involvement. This may be because parents who felt more frustrated by their children were less inclined to volunteer in the classroom. However, it was not clear why they did not avail themselves of workshops or Social Services (see data reported later) in order to help address these feelings.

The finding that engagement in self-sufficiency activities was associated with less involvement was consistent with our pilot study. In that study, parents who were working, going to school or volunteering outside Head Start, those reporting a schedule that conflicted with Head Start activities, and those who worked during the day participated less. Together, the findings of the two studies are suggestive that parents' engagement in selfsufficiency activities needs to be considered in studying the impact of parent involvement in Head Start.

At pretest, $40 \%$ of the parents reported a large number and frequency of depressive symptoms indicative of psychological distress (e.g., Coin, 1994). ${ }^{35}$ However, neither parents' depressive symptoms nor their feelings of personal control predicted any of the measures of participation. ${ }^{36}$ The attrition data also indicated that depression was not a factor influencing who dropped out of Head Start, indicating that these mothers did not behave like middle-class parents (Radloff, 1979). Greater depressive symptomatology did not decrease their level of activity, at least with respect to Head Start, suggesting remarkable personal strength and resilience, a dedication to the educational futures of their young children, and a confidence in Head Start as a vehicle to help them.

The number of predictors associated with attendance at workshops and policy meetings was low, less than that expected by chance. This suggested the need of a theoretically-based model that specifies the types of variables which would be likely to predict specific types of parent involvement. There are many other types of variables which

\footnotetext{
35 Others (e.g., Radloff, 1979) considered scores of 16 and above to indicate clinical depression.

${ }^{36}$ In the barriers survey, $47 \%$ of the parents reported that they often felt sad, down or depressed, showing a trend towards less staff-judged participation. However, this was not replicated in the longitudinal study. This may be due to differences in the two measures of "depression". In the barriers study, the measure tapped only one type of depressive symptom, parents' feelings of sadness and depression. In the longitudinal study, a large range of bodily and other symptoms was assessed.
} 
may affect parents' participation in Head Start but which were not measured in the present study. Among these are: other family and community obligations of the parent, characteristics of the Head Start program, Head Start teacher characteristics, the skills and preferences of the parents, specific requests from the Head Start teacher and social service worker, and significant events (e.g., death in the family, separation) that may occur during the Head Start year. Other aspects of parent involvement which may be predicted by demographic, personal, and contextual factors are changes in level, type and quality of participation over the course of the Head Start year.

\section{Parent Involvement and Parent Outcomes}

Objective 2, to examine the relationship between parent participation in Head Start and the parents themselves, the Head Start children, and siblings is addressed in the following sections.

- Is parent participation in Head Start positively related to positive parent-child relationships?

Parents were assessed on four aspects of the parent-child relationship -- parental encouragement of the child's independence, strictness, aggravation, and frequency of daily parenting hassles -- at both the beginning and the end of the Head Start year. The intercorrelations among the four measures ranged from -.01 to +.55 .

At pretest, the parents reported moderately positive parent-child relations. Parents' pretest and posttest scores were compared to determine if the sample as a whole changed over the course of the Head Start year (see Table 7). There were no changes in parents' scores on three of the variables. However, mean parental encouragement of the child's independence increased statistically significantly from pretest to posttest, indicating that parents were more facilitative of their children's independence at the end of the Head Start year, likely due in part to the child's maturation.

In order to test the hypothesis of a positive association of involvement and the parent-child relationship variables, partial correlations were computed between the three measures of Involvement (Undifferentiated Involvement, and the two activity types -Volunteering and Workshops/Policy Meetings) and the four aspects of the parent-child relationship, controlling for pretest scores (see Table 8 ). The outcomes with statistically 
significant relationships or trends towards statistical significance (encouragement of the child's independence, strictness, frequency of daily parenting hassles) then were subjected to regression analyses which controlled for demographic factors related to the outcome as well as for the pretest. The results of the regression analyses are reported below. As none of the partial correlations between the involvement variables and aggravation were statistically significant, exploratory analyses were conducted on this outcome that also are reported below.

\section{Association of Parent Involvement with Parental Encouragement of the Child's} Independence

As the partial correlation tended towards statistical significance (see Table 8), a regression of parental encouragement of the Head Start child's independence on Undifferentiated Involvement was conducted, controlling for the pretest and Head Start child's gender, the one demographic variable correlated with the outcome [parents of boys reported more encouraging attitudes than parents of girls $(r=.16, p<.05)]$. In support of the hypothesis, after controlling for gender and the pretest, Undifferentiated Involvement showed a trend towards a statistically significant positive relationship with parental encouragement of the child's independence ( $\mathrm{r}$ square change $=.02, \mathrm{p}<.10$ ).

As the partial correlation between Workshops/Policy Meetings and the outcome also tended towards statistical significance, a regression was conducted which controlled for gender and the pretest. Consistent with the hypothesis, more Workshops/Policy Meetings showed a trend towards a statistically significant association with greater parental encouragement of the child's independence ( $\mathrm{r}$ square change $=.02, \mathrm{p}<.10$ ).

\section{Association of Parent Involvement with Parental Strictness}

As the partial correlation was statistically significant, a regression of parental strictness on Undifferentiated Involvement was conducted, controlling for the pretest and mother's education and age, the two demographic variables which were correlated with the outcome. [The lower the mother's educational level, the stricter the parent was with her child $(r=-.15, p<.05)$, and having an older Head Start child tended towards a statistically significant association with greater posttest strictness $(r=.13, p<.10)]$. In support of the 
hypothesis, after controlling for mother's education and the Head Start child's age, Undifferentiated Involvement was marginally statistically significantly associated with less parental strictness ( $\mathrm{r}$ square change $=.02, \mathrm{p}<.06$ ).

As the partial correlation between Workshops/Policy Meetings and strictness tended towards statistical significance, a regression was conducted which controlled for mother's education and the Head Start child's age. Consistent with the hypothesis, after controlling for the pretest and demographic variables, more Workshops/Policy Meetings showed a trend towards a statistically significant association with less parental strictness ( $\mathrm{r}$ square change $=.01, \mathrm{p}<.10)$.

\section{Association of Parent Involvement with Frequency of Daily Parenting Hassles}

As the partial correlation tended towards statistical significance, a regression of frequency of daily parenting hassles on Undifferentiated Involvement was conducted, controlling for the pretest and number of other children in the home, the one demographic factor correlated with frequency of daily parenting hassles (the more children at home, the greater the frequency of daily parenting hassles, $r=.23, \mathrm{p}<.004)$. In support of the hypothesis, after controlling for number of other children in the home and the pretest, Undifferentiated Involvement showed a trend towards a statistically significant relationship with daily parenting hassles ( $\mathrm{r}$ square change $=.01, \mathrm{p}<.07$ ) such that more involvement was associated with less parenting hassles.

The partial correlations between the two types of involvement activities (Volunteering, Workshops/Policy Meetings) and posttest daily parenting hassles were not statistically significant.

\section{Association of Parent Involvement with Parenting Aggravation}

Because there was no evidence of a linear relationship between any of the three involvement variables and parenting aggravation, three additional post-hoc analyses were performed to explore possible complex relationships. The exploratory analyses were performed on the Undifferentiated Involvement variable.

The first analysis explored the possibility of a non-linear relationship between level of Undifferentiated Involvement and parental aggravation. For this analysis, Undifferentiated 
Involvement was operationalized as a categorical independent variable having three levels: low, medium, and high. The second analysis addressed the possibility that controlling for demographic, personal, and contextual variables related to the outcome would increase the precision of the analysis, revealing a positive relationship between involvement and aggravation. The last analysis examined the possibility that a third variable -- engagement in self-sufficiency promoting activities during the Head Start year -- moderated the relationship between Undifferentiated Involvement and aggravation. None of the analyses were statistically significant.

Summary and Discussion of the Association of Parent Involvement with the Parent-Child Relationship

There was modest support for the hypothesis that parent involvement in Head Start is positively related to a positive parent-child relationship as measured by parental encouragement of the child's independence, parental strictness, and the frequency of daily parenting hassles. After controlling for demographic factors and pretest differences, parents who participated more in Head Start also: 1) reported marginally significantly decreased parental strictness, 2) had a greater tendency to encourage their child's independence, and 3) showed a tendency towards experiencing less frequent daily parenting hassles.

These findings have extended research on parent involvement in Head Start by suggesting that parent involvement is related to important aspects of the parent-child relationship. Although the results were modest, the findings are consistent with the hypothesis that parent involvement is related to better outcomes for parents and children even after controlling for pretest differences and important demographic factors. Several possible explanations were explored for the lack of a relationship between Undifferentiated Involvement and aggravation, but none explained the statistically nonsignificant findings. ${ }^{37}$

The results also suggest that specific types of participation may have different benefits for the parent-child relationship. More Workshops and Policy Meetings tended towards statistically significant associations with both greater encouragement of the child's

\footnotetext{
${ }^{37}$ There was a significant positive impact of Involvement on aggravation in Agency Two, to be reported with the Agency Two results.
} 
independence and with less parental strictness. These results can be interpreted in light of the goals of the workshops and policy meetings to improve parenting skills. Participating in policy meetings throughout the year provides parents with opportunities to be respected for their independent thinking and assertiveness in a group, which may translate to more respectful attitudes towards their own child's independent thinking. Thus, these activities may provide the types of skills and experiences they aim to. We were surprised to see that the second type of involvement activity -- Volunteering -- did not exhibit a statistically significant association with these aspects of the parent-child relationship. However, because the relationships were in the same direction as for Undifferentiated Involvement, Volunteering may have contributed to the relationships between Undifferentiated Involvement and these outcomes. The inclusion of office and kitchen work in the volunteering variable, made necessary because these activities were not recorded separately at the Head Start centers, may have attenuated the effects of classroom volunteering on the parent-child relationship.

Although multiple aspects of the parent-child relationship were measured in this study, there remain many other aspects that were not assessed that might also be influenced by attitudes and skills learned from the Head Start parent involvement activities. Future research can build on the findings of the present study by examining other outcomes in order to continue to develop a comprehensive picture of the benefits of Head Start for parents and children. The findings of the present study are encouraging because positive parent-child relationships are central to the child's well-being and successful school functioning and thus suggest potential indirect pathways through which parent involvement benefits children.

- Is parent participation in Head Start positively related to the home learning environment?

Parents' structuring of three aspects of the home learning environment were measured at the beginning and end of the Head Start year: 1) number of school readiness skills parent helped the Head Start child learn; 2) number of educationally relevant play materials in the home and 3) parent's expectation for the Head Start child's school performance. At pretest, parents reported helping their children learn a moderate number of important school readiness skills and provided a moderate number of play materials in 
the home (see Table 7). Parents reported high expectations for their child's future school achievement. For the sample as a whole, two of the three variables showed statistically significant change over the Head Start year: The number of school readiness skills the parent helped her child learn and the number of play materials in the home both increased.

Partial correlations were computed between the three involvement variables and the three home learning environment variables (see Table 8). The relationship which was statistically significant (helping the child learn school-readiness skills) and the relationship which showed a trend towards statistical significance (parental expectations for the child's school success) were submitted to regression analyses which controlled for demographic factors related to the outcome and for the pretest. These are reported below. As the partial correlations for play materials were not statistically significant, exploratory analyses were conducted on this outcome. These also are reported below.

\section{Association of Parent Involvement with the Number of School-Readiness Skills the Parent Helped her Child Learn}

As the partial correlation was significant, a regression of number of school-readiness skills on Undifferentiated Involvement was conducted, controlling for the pretest and four demographic variables which were associated with this outcome: Having an older Head Start child $(\mathrm{r}=.16, \mathrm{p}<.05)$, and having a girl $(\mathrm{r}=-.20, \mathrm{p}<.009)$ were statistically significantly associated with the parent helping her child learn more school-readiness skills. Having less other children in the home $(r=-.15, \mathrm{p}<.06)$ was marginally statistically significantly associated with more parental help, and being an older mother $(\mathrm{r}=.14, \mathrm{p}<.10)$ tended towards a statistically significant positive association. In support of the hypothesis, after controlling for these demographic variables and the pretest, greater Undifferentiated Involvement was statistically significantly associated with the parent's helping her child learn more school-readiness skills ( $\mathrm{r}$ square change $=.04, \mathrm{p}<.002$ ).

As the partial correlations between both activity types and the outcome were statistically significant, a regression was conducted for each activity type which controlled for the demographic factors and the pretest. Consistent with the hypothesis, both activity types -- Volunteering and Workshops/Policy Meetings -- were significantly and positively 
associated with the parent helping her child learn more school-readiness skills [ $\mathrm{r}$ square change for Volunteering was .04, $\mathrm{p}<.002$; for Workshops/Policy Meetings, $\mathrm{r}$ square change was $.03, \mathrm{p}<.01]$. Regression analyses varying the order of entry of the two activity types were conducted to explore the association of each variable with the outcome over and above the association of the other. The results showed that Volunteering remained a statistically significant predictor of the outcome with Workshops/Policy Meetings controlled for in the model. Workshops/Policy Meetings was no longer statistically significant with Volunteering in the model.

\section{Association of Parent Involvement with Parents' Expectations for their Head Start Child's $\underline{\text { School Success }}$}

Because none of the demographic factors was statistically significantly associated with parents' expectations for the Head Start child's school success, the partial correlation controlling for the pretest constituted the test of the hypothesis. Although Undifferentiated Involvement was not statistically significantly correlated with the outcome, one of the activity types -- Volunteering -- tended towards a positive and statistically significant association with greater parental expectations for the child's school success $(r=-.15$, $\mathrm{p}<.10) .^{38}$

\section{Association of Parent Involvement with Parent's Provision of Educationally Relevant Play Materials in the Home}

Because there was no evidence of a linear relationship between involvement and parents' provision of play materials, three additional analyses were performed to explore possible complex relationships.

The first analysis revealed tentative evidence of a non-linear relationship between Undifferentiated Involvement, measured categorically, and the outcome. There were no group differences at the beginning of the Head Start year, but the analysis of covariance of posttest play materials controlling for pretest tended towards statistical significance

\footnotetext{
${ }^{38}$ The correlation is negative because expectations was reverse-coded. A smaller score indicates higher expectations.
} 
$[F(1,161)=2.56, p<.10]$ and the repeated measures test of the change from pretest to posttest for the three levels of Undifferentiated Involvement was marginally statistically significant $[\mathrm{F}(2,162=2.96, \mathrm{p}<.055]$. Inspection of both adjusted and unadjusted posttest means indicated that medium involved parents provided the greatest number of play materials, followed by the high involved parents, at the end of the Head Start year. The low involved parents provided the least number of play materials.

The second analysis (covariate regression analysis) addressed the possibility that controlling for demographic, personal, and contextual variables which were related to the outcome would increase the precision of the analysis, revealing a positive relationship between involvement and play materials. This analysis was not statistically significant.

The next analysis examined the possibility that an interaction between Undifferentiated Involvement and engagement in self-sufficiency promoting activities during the Head Start year affected parent's provision of play materials. The analyses on the interaction were not statistically significant and follow-up analyses did not reveal a consistent pattern of effects.

\section{Summary and Discussion of the Association of Involvement with the Home Learning Environment}

There was modest support for the hypothesis that parent involvement in Head Start is positively related to parents' providing a positive home learning environment. After controlling for demographic factors and the pretest, parents who participated more overall also helped their child learn statistically significantly more school-readiness skills. Thus, in addition to the school readiness training in the Head Start child's classroom, Head Start parent participation may provide an additional opportunity for children to learn and reinforce the skills with their parents at home, resulting in a potentially stronger foundation for children's entering school and being perceived by their teachers as competent. This is. an important finding since number of skills with which a child enter kindergarten may influence the teacher's initial impression of the child's competence. This first impression then may influence the child's long-term school success by creating a self-fulfilling prophecy in which the initial positive impression elicits teacher responses that result in further 
improvements in the child's performance and further strengthening of the teacher's judgment that the child is competent (Alexander \& Entwisle, 1988).

The two activity types -- Volunteering and Workshops/Policy Meetings -- had statistically significant positive associations with parents' helping the child learn schoolreadiness skills, after controlling for demographic factors and the pretest. Volunteering remained a statistically significant predictor of the outcome when attendance at Workshops and Policy Meetings was controlled. One possible explanation for this finding is that Volunteering in the classroom may have provided concrete experience with developmentally appropriate readiness skills and effective instructional techniques, as well as individualized information for each parent about the skills that her Head Start child needed to learn.

There was tentative evidence that one of the types of involvement activities -Volunteering -- was associated with improvements in parents' expectations for the Head Start child's long-term school success. More Volunteering tended towards a statistically significant association with higher parental expectations. One plausible explanation for this pattern of findings is that parents who volunteered more in the classroom had the opportunity to directly observe their child's performance, providing additional positive information about the child's capabilities. Therefore, volunteering in the classroom may provide multiple benefits -- help for the teacher, and raised expectations of the parent for her child. Research has shown that higher parental expectations are positively associated with her child's school performance (Clark, 1993; Keith, Keith, Troutman, Bickley, Trivette, \& Singh, 1993; Miller, Manhal, \& Mee, 1991; Phillips, 1992; Reynolds \& Gill, 1994;

Schiamberg \& Chin, 1986; Wang \& Wildman, 1994).

There was tentative evidence that number of play materials in the home was nonlinearly related to extent of Undifferentiated Involvement. ${ }^{39}$ The medium involved parents tended to improve the most, but the reason for this is not apparent.

${ }^{39}$ However, at Agency Two, more Volunteering was associated with the provision of more play materials. 
- Is parent participation in Head Start positively related to parental well-being?

Two aspects of parents' well-being: 1) personal control/mastery; and 2) depression; were assessed both at the beginning and at the end of the Head Start year. Parents began the Head Start year with a moderately high degree of perceived control over their lives. In contrast, parents' reported relatively high levels of symptoms indicative of psychological distress (Coin, 1994; Radloff, 1979). ${ }^{40}$

Both personal control and depression showed statistically significant improvement from pretest to posttest, indicating that parents felt more in control of their lives and were experiencing less depressive symptomatology at the end of the Head Start year. However, the partial correlations revealed no statistically significant linear relationships or trends in the expected direction between the three involvement variables and the two outcomes. Therefore, additional analyses were conducted that explored possible complex relationships. The results of these analyses are reported below:

\section{Association of Parent Involvement with Personal Control}

The possibility of a non-linear relationship between Undifferentiated Involvement, measured categorically, and personal control was explored. The second analysis (covariate regression analysis) addressed the possibility that controlling for demographic, contextual, and personal variables measured at pretest which were related to the outcome would increase the precision of the analysis, revealing a positive relationship between involvement and personal control. The next analysis examined the possibility that a third variable, engagement in self-sufficiency promoting activities during the Head Start year, moderated the relationship of Undifferentiated Involvement with personal control. None of the analyses were statistically significant. ${ }^{41}$

\footnotetext{
${ }^{40}$ Recent research on depression in minority populations has found that Hispanic adults have a higher mean level of depressive symptomology than white adults (Golding \& Aneshensel, 1989).

41 There was evidence of a relationship between engagement in self-sufficiency activities during the Head Start year and personal control, independent of involvement. Parents who engaged in self-sufficiency promoting activities, compared to non-engaged parents, began the Head Start year with greater feelings of personal control $[F(1,163)=4.57, p<.05]$. At the end of the year, these parents also reported greater feelings of personal control, even after controlling for pretest differences $[F(1,163)=13.07, p<.001]$. Thus, engagement in self-sufficiency promoting activities was associated with greater personal control.
} 


\section{Association of Parent Involvement with Depression}

Because there was no evidence of a linear relationship between involvement and depression, additional analyses were performed to explore possible complex relationships.

First, the possibility of a non-linear relationship between Undifferentiated Involvement, measured categorically, and depression was explored. There was no evidence of a non-linear relationship, but there was evidence that less involvement, measured categorically, was associated with less depressive symptomatology. An analysis of covariance on posttest depression scores controlling for pretest depression tended towards statistical significance $[\mathrm{F}(2,161)=2.37, \mathrm{p}<.10]$. At posttest, the high involved parents tended to experience the most depressive symptoms, followed by the medium, and then the low involved parents. This trend remained after controlling for pretest depression scores. Thus, contrary to expectation, more involved parents tended to have more depressive symptomatology.

The second analysis (covariate regression analysis) addressed the possibility that controlling for demographic, contextual, and personal variables (see Table 1) which were related to the outcome would increase the precision of the analysis, revealing a positive relationship between involvement and depression. This analysis was not statistically significant.

The possibility was addressed that engagement in self-sufficiency promoting activities during the Head Start year moderated the relationship of Undifferentiated Involvement and depression. Post-hoc examination of the change from pretest to posttest for the six involvement by engagement groups (see Figure 1) suggested that all of the groups improved over the Head Start year except the high involved engaged group, who became more depressed.

Because this pattern was potentially important for theory and for policy, a regression analysis was conducted to explore the interaction using the continuous measure of Undifferentiated Involvement. Although regression analyses of interactions are harder to interpret, they have the advantage of minimizing the loss of information in the independent variable which occurs when using categorical variables. A hierarchical multiple regression analysis was performed in which the demographic and other predictor variables associated with posttest depression were entered in Block 1, followed by the pretest in Block 2, 
engagement in self-sufficiency activities in Block 3, Undifferentiated Involvement in Block 4, and a term representing the interaction of "engagement" and Undifferentiated Involvement in Block 5. The interaction term tended towards statistical significance (Beta $=-.15, \mathrm{p}<.07)$, indicating that for parents engaged in self-sufficiency promoting activities outside Head Start, more than average involvement (above 97 hours over the Head Start year) tended to result in more depressive symptoms. In contrast, for not engaged parents, more than average involvement tended to result in less depressive symptoms.

\section{Summary and Discussion of the Association of Involvement with Parental Well-Being}

There was tentative evidence that engagement in self-sufficiency promoting activities during the Head Start year moderated the effects of involvement on depressive symptomatology. Engaged parents who were more involved tended to report more frequent and/or more depressive symptomatology. Although these parents reported feeling a fair degree of personal control, their increased depressive symptomatology may indicate overload or anxiety about their activities. At least one other current study has found a similar pattern of increased depressive symptomatology related to high levels of activity (personal communication with Fern Marx, Yale University, June, 1995).

A clear pattern did not emerge with respect to the relationship between involvement and parents' feelings of personal control. Future research should concentrate on the development of a causal model that identifies potentially important variables (e.g., engagement in self-sufficiency promoting activities) which, along with involvement, influence different aspects of well-being.

- Is parent participation in Head Start positively related to parents' behaviors that promote socioeconomic self-sufficiency during the year following Head Start?

Data were collected on a subsample of 115 parents' engagement in three types of self-sufficiency promoting activities during the year following Head Start: 1) attending school or participating in a job-training program; 2) not being pregnant, and 3) working at a paid job. Although having a paid job normally means that a family is self-sufficient, the income of these working Head Start families still hovered near the poverty level -- of the 31 families who were working during the follow-up year, $45 \%$ were also receiving public assistance. 
Although pretest data were not available on parents' activities at the beginning of the Head Start year, data were available on their activities during the Head Start year: $21 \%$ of this subsample of parents were attending school or participating in job training, $87 \%$ were not pregnant, and $12 \%$ had a paid job during that year. During the follow-up year, $60 \%$ of the parents were attending school or participating in some type of job training, $90 \%$ were not pregnant, and $27 \%$ had a paid job.

For the sample as a whole, it was of interest to assess whether the percentage of parents engaging in each of the self-sufficiency activities changed from the Head Start year to the follow-up year. There were statistically significant increases in the proportions of parents in education/job training and in paid employment. Thus, there was an overall increase in parents' self-sufficiency promoting activities which may have been due in part to the institution of the "welfare to work" requirements during the follow-up year.

Because the outcomes were dichotomous, logistic regression analyses were used to test the hypotheses. Table 8 shows the odds ratios that link increases in involvement to changes in the probability of each self-sufficiency outcome. In our data set, the analyses in Table 8 predicted the odds of not having attended school or participated in job training, not having been pregnant, and not having had a job during the year after Head Start. For each outcome, a logistic regression was conducted with one of the three involvement variables as the predictor. Those relationships which were statistically significant or tended towards statistical significance (not being pregnant and Undifferentiated Involvement and Volunteering) were subjected to logistic regression analyses which also controlled for demographic factors related to the outcome, and are reported below. As there were no statistically significant relationships between any of the involvement variables and the other two outcomes (paid employment and education/job training), additional exploratory analyses were conducted on these outcomes. These also are reported below.

\section{Association of Parent Involvement with Not being Pregnant During the Year Following Head Start}

As the initial logistic regression of "not pregnant" on Undifferentiated Involvement tended towards statistical significance, a second logistic regression was conducted which 
controlled for mother's age -- the one demographic variable correlated with the outcome (older mothers were less likely to have been pregnant $[\operatorname{Exp}(B)=1.19, \mathrm{p}<.01]$ ). In support of the hypothesis, after controlling for mother's age, there was a statistically significant increase in the likelihood of not being pregnant $[\operatorname{Exp}(B)=1.32, p<.05]$ related to more Undifferentiated Involvement.

As the initial logistic regression of "not pregnant" on Volunteering tended towards statistical significance, a second regression was conducted with this involvement variable controlling for mother's age. Consistent with the hypothesis, there was a statistically significant increase in the likelihood of not being pregnant $[\operatorname{Exp}(B)=1.25, p<.05]$ during the follow-up year related to more Volunteering.

\section{Association of Parent Involvement with Having Paid Employment During the Year} Following Head Start

Because Involvement was not statistically significantly associated with paid employment during the follow-up year, additional analyses were performed to explore more complex relationships.

The first analysis explored the possibility of a relationship between Undifferentiated Involvement, measured categorically, and the outcome. The second analysis (covariate regression analysis) addressed the possibility that controlling for variables related to the outcome would increase the precision of the analysis, revealing a positive relationship between involvement and having paid employment. There was no evidence for either effect.

A third analysis examined the possibility that parents' paid employment during the Head Start year, together with Undifferentiated Involvement, predicted paid employment during the follow-up year. As reported earlier; parents engaged in self-sufficiency promoting activities during the Head Start year were less involved than non-engaged parents. Although the direction of causality linking involvement and having a paid job could not be determined, it seemed plausible that job status during the Head Start year might predict job status during the follow-up year, and that with job status during the Head Start year controlled in the model, Undifferentiated Involvement then might be associated with paid employment during the follow-up year. Therefore, an additional exploratory logistic regression analysis was conducted in which employment status (yes vs. no) and 
Undifferentiated Involvement were entered together as predictors. Consistent with the hypothesis, with job status during the Head Start year controlled for in the model, ${ }^{42}$ greater Undifferentiated Involvement tended to decrease the odds of not being employed during the follow-up year $[\operatorname{Exp}($ Beta $)=.84, \mathrm{p}<.10]$.

\section{Association of Involvement with Participation in Education or Job Training During the Follow-up Year}

As there was no relationship between any of the involvement variables and participation in education/job training during the follow-up year, additional exploratory analyses were conducted. The analyses were not statistically significant. An analysis exploring the relationship between Undifferentiated Involvement and education/job training during the follow-up year while controlling for education/job training activities during the Head Start year also was not statistically significant.

\section{Summary and Discussion of the Association of Parent Involvement with Engagement in Self-Sufficiency Promoting Activities}

There was tentative support for the hypothesis that parent involvement in Head Start is associated with parents' greater engagement in self-sufficiency promoting activities during the year following Head Start. Greater Undifferentiated Involvement and more hours of one of the activity types -- Volunteering -- tended to increase the likelihood of not being pregnant during the year following Head Start. In addition, more Undifferentiated Involvement tended to significantly increase the odds of having paid employment during the follow-up period after taking into account paid employment during the Head Start year. In contrast, there was no evidence that involvement in Head Start was linked with participation in education/job training activities in the follow-up year.

The finding that more overall involvement and more volunteering are associated with a greater likelihood of not being pregnant during the year following Head Start is

\footnotetext{
42 As expected, having paid employment during the Head Start year was also a significant predictor of having paid employment during the follow-up year.
} 
particularly important in the current harsh economic climate. In a time of shrinking social resources and job opportunities (e.g., difficulty obtaining health insurance, less financial aid to families with dependent children), not being pregnant removes one major source of physical, emotional, and financial stress for the entire family. A mother who is not pregnant may have more psychological and physical resources available to care for her current family, and to deal with other major life challenges, e.g., marriage, parenting, employment; education, and other life stresses. In addition, the entire family benefits from not having the tremendous expenses associated with pregnancy, childbearing and raising an additional child.

Our findings also are very encouraging because our design dictated a conservative test of the hypothesis. Institution of the welfare-to-work law during the follow-up year may have weakened the effect of involvement because parents, irrespective of their level of Head Start involvement, were required to engage in at least one of these activities to continue receiving income support. Nonetheless, we found that more parent involvement in Head Start was linked with greater participation in self-sufficiency promoting activities. This finding should be of interest to policy makers concerned with increasing the economic selfsufficiency of poor families. These findings are also important because engagement in selfsufficiency activities affects not just the parent but the entire family. All of the family members will benefit from increased income due to a parent's employment. The findings also suggest continued effects of participation in Head Start one year after the end of the program. This demonstrates the ability of Head Start to have an impact after the family has completed the program.

- Is parent participation in Head Start positively related to parents' involvement in their Head Start child's education in elementary school?

Teacher ratings of the parents' overall involvement and interest in their child's kindergarten school experience indicated that on the average, parents frequently participated and showed interest in their child's education. In contrast, teachers reported that over $60 \%$ of the parents never or rarely helped or offered to help in the classroom. It was not possible, from the data available in the present study, to ascertain whether 
sufficient opportunities to engage in these behaviors were made available to parents by the school.

In order to test the hypothesis of a positive association between parent involvement in Head Start and parent involvement in elementary school, zero-order correlations were computed between the three involvement in Head Start variables and the two involvement in kindergarten scales. One of the correlations was statistically significant and the other tended towards significance: More Undifferentiated Involvement tended towards a statistically significant association with greater overall involvement in the Head Start child's kindergarten experience $(\mathrm{r}=.16, \mathrm{p}<.10)$; and greater attendance at workshops and policy meetings was statistically significantly associated with more parental help in the kindergarten classroom and/or more offers of help $(\mathrm{r}=.22, \mathrm{p}<.05)$. These two relationships were subjected to hierarchical regression analyses which controlled for demographic factors related to the outcome. The results of these analyses are reported below:

\section{Association of Parent Involvement in Head Start with Parents' Overall Involvement in the Kindergarten Classroom}

As the correlation between Undifferentiated Involvement and Overall Involvement in kindergarten tended towards significance, a regression was conducted that controlled for child's age -- the demographic factor related to the outcome (parents of younger children tended to be more involved, $\mathrm{r}=-.17, \mathrm{p}<.10)$. After controlling for child's age, Undifferentiated Involvement in Head Start was no longer significantly related to parents' overall involvement in kindergarten. ${ }^{43}$

\section{Association of Parent Involvement in Head Start with Parents' Helping in the Kindergarten} Classroom

None of the demographic factors were significantly associated with parents' helping in the kindergarten classroom. Therefore a regression analysis controlling for demographic

\footnotetext{
${ }^{43}$ With both variables in the equation, the model was only marginally statistically significant, and neither age nor involvement was statistically significant.
} 
factors was not conducted, and the statistically significant zero-order correlation constituted the test of the hypothesis. More Workshops/Policy Meetings was statistically significantly associated with greater parental helping/and or offers to help in the kindergarten classroom $(\mathrm{r}=.22, \mathrm{p}<.05)$.

\section{Summary and Discussion of the Association of Parent Involvement in Head Start with} Parents' Involvement in Elementary School

There was limited support for the notion that greater parent involvement in Head Start is associated with greater parent involvement in public school: Attendance at Workshops/Policy Meetings was associated with parents' offering more help and/or helping more in the kindergarten classroom. This finding suggests that parents may feel more willing to offer help when they have more knowledge of appropriate practices for young children and child development milestones, the underlying content of many of the workshops in Head Start. Similarly, attending more policy committees and meetings might give them more confidence to approach teaching staff.

A significant direct relationship between overall involvement in Head Start and overall involvement in kindergarten was not found. However, parents may have been more involved with their child's education at home or in other ways that were not captured by these data.

\section{Parent Involvement and Child Outcomes}

- Is parent participation in Head Start positively related to the social competence of the Head Start child?

Five aspects of the Head Start child's social competence were assessed, three at the end of the Head Start year, and two at the end of the Head Start child's kindergarten year.

At the end of the Head Start year, parents rated the Head Start child's disruptiveness/demandingness, and cooperativeness/compliance (cooperativeness) at home. At the same time, the Head Start child's teacher administered to the Head Start child the CPI school-readiness test. For the sample as a whole, there were statistically significant gains on all three variables over the Head Start year (see Table 9). 
In order to test the hypothesis of a positive association between involvement and these three child outcome variables, partial correlations were computed, controlling for the pretest (see Table 10). The relationship that was statistically significant (cooperativeness) was then subjected to regression analysis which controlled for demographic factors related to the outcome as well as for the pretest. The results are reported below. As the partial correlations between involvement and the other two outcomes (disruptiveness and CPI) were not statistically significant, additional exploratory analyses were conducted on these outcomes, and also are reported below.

\section{Association of Parent Involvement with the Head Start Child's Cooperativeness at Home}

As the partial correlation was statistically significant, a regression of cooperativeness at home on Undifferentiated Involvement was conducted, controlling for the pretest and Head Start child's gender, the one demographic variable that tended towards a statistically significant correlation with the outcome (girls tended to be perceived by their parents as more cooperative than boys $(\mathrm{r}=-.13, \mathrm{p}<.10)$. In support of the hypothesis, after controlling for gender and the pretest, Undifferentiated Involvement was statistically significantly and positively correlated with greater Head Start child cooperativeness at home (rsq change $=.028, \mathrm{p}<.02)$.

The next set of analyses explored the relationship between type of involvement activity -- Volunteering and Workshops/Policy Meetings -- and Head Start child's cooperativeness at home (see Table 10). After controlling for gender and the pretest, Volunteering was marginally statistically significantly correlated with cooperativeness (rsq change $=.019, \mathrm{p}<.055)$ and Workshops/Policy Meetings tended towards a statistically significant positive correlation (rsq change $=.018, \mathrm{p}<.07$ ).

\section{Association of Parent Involvement with the Head Start Child's Disruptiveness at Home}

There was no evidence of a linear relationship between Involvement and the Head Start child's disruptiveness at home (see Table 10). ${ }^{44}$ Therefore, several additional analyses were performed to explore possible complex relationships.

\footnotetext{
${ }^{44}$ The lack of significant findings may at least in part be due to the low reliability of the scale (Cronbach's alpha $=.47)$.
} 
Prior to the planned exploratory analyses, we examined the relationship between disruptiveness and cooperativeness. Because disruptiveness and cooperativeness can be seen as somewhat opposite behaviorally, and because cooperativeness was associated with involvement, we were interested in the correlation between them. Interestingly, for this sample of mostly (92\%) Dominican mothers, there was no relationship between the two variables, suggesting that these Dominican parents did not perceive these two behavior patterns to be mutually exclusive. ${ }^{45}$

The next analysis explored the possibility of a non-linear relationship between Undifferentiated Involvement, measured categorically, and the Head Start child's disruptiveness at home. Another analysis addressed the possibility that controlling for demographic, contextual, and personal variables which were related to the outcome would increase the precision of the analysis, revealing a positive relationship between involvement and disruptiveness. These analyses; however, were not statistically significant.

The analyses examining the interaction between engagement in self-sufficiency promoting activities during the Head Start year and Undifferentiated Involvement were not statistically significant either and follow-up analyses did not reveal a consistent pattern of relationships. For example, although the high involved engaged parents reported their children to be less disruptive at pretest, there were no statistically significant group differences in amount of change over the Head Start year or on posttest scores.

\section{Association of Parent Involvement with the Head Start Child's CPI School-Readiness Score}

There was no evidence of a linear relationship between Involvement and the Head Start child's CPI score (see Table 10). Therefore, additional analyses were performed to explore possible complex relationships.

The first analysis addressed the possibility of a non-linear relationship between Undifferentiated Involvement, measured categorically, and the outcome, but there was no evidence of this relationship. The second analysis addressed the possibility that controlling for demographic, contextual, and personal variables which were related to the outcome

\footnotetext{
${ }^{45}$ The relationships between disruptiveness and cooperativeness ratings by non-Dominican parents at Agency Two $(r=-.58, p<.001)$ and by non-Dominican Agency One teachers $(r=-.60, p<.001)$ were negative.
} 
would increase the precision of the analysis, revealing a positive relationship between involvement and CPI scores. This analysis also was not statistically significant.

There was evidence of an interaction between engagement in self-sufficiency promoting activities during the Head Start year and Undifferentiated Involvement on the Head Start child's CPI scores (see Figure 2). The interaction of engagement and involvement on posttest CPI scores, controlling for pretest, was statistically significant $[F(2,112)=3.40, p<.04]$. The six involvement by engagement groups did not differ at pretest, but did differ at posttest. When comparing the three involvement groups for engaged parents, children of the medium involved parents had the highest CPI scores. Among families with non-engaged parents, children of the high involved parents had the highest CPI scores, and children of the medium involved parents had the lowest. This pattern suggests that the total amount of activity the parent is engaged in may be an important variable that may affect the child's development as well as the parent's. High involvement in Head Start and engagement in self-sufficiency promoting activities outside of Head Start may lead to stress on the part of the parent which adversely affects the school readiness of the child. In contrast, when parents are not engaged outside of Head Start, high involvement results in the child's having the best school-readiness scores.

Two additional aspects of the Head Start child's social competence were assessed at the end of the kindergarten year. The Head Start child's kindergarten teacher rated the child's: 1) overall adaptation to the kindergarten classroom; and 2) present and anticipated future performance. On average, the Head Start children were rated somewhat better than moderate on both scales. To test the hypothesis of a positive association between involvement in Head Start and the Head Start child's kindergarten functioning, zero order correlations $s^{46}$ were computed between the three measures of involvement and the two post-kindergarten outcomes. There were significant relationships between the involvement variables and both outcomes (see Table 10). These were then subjected to regression analyses which controlled for relevant demographic factors. The results of these analyses are reported below.

\footnotetext{
${ }^{46}$ Zero order correlations were computed instead of partial correlations because pretest teacher ratings were not available since this was the children's first experience in public school.
} 
Association of Undifferentiated Involvement with the Head Start Child's Adaptation to the Kindergarten Classroom

As the correlations between the three involvement variables and adaptation to the classroom were statistically significant or tended towards significance, three regression analyses were conducted which each controlled for two statistically significant demographic factors related to the outcome -- the Head Start child's gender and pretest CPI schoolreadiness scores. (Being female and having greater initial CPI scores were associated with teacher ratings of better adaptation to the kindergarten classroom.) However, after controlling for gender and pretest CPI score, the three involvement variables were no longer. statistically significantly related to teacher ratings of the Head Start child's adaptation to the kindergarten classroom. ${ }^{47}$

\section{Association of Parent Involvement with Kindergarten Teacher Ratings of the Head Start Child's Present and Anticipated Future Performance}

As the zero-order correlation between Undifferentiated Involvement and the outcome was marginally statistically significant, a regression was conducted that controlled for the pretest and the two demographic variables related to the outcome -- gender and preHead Start CPI. As the correlation between Volunteering and the outcome was significant, a second regression was conducted which also controlled for gender and pre-Head Start CPI. With the two demographic variables in the model, neither Undifferentiated Involvement nor Volunteering were statistically significantly associated with kindergarten teacher ratings of the Head Start child's current and anticipated future performance.

Summary and Discussion of the Association of Parent Involvement with the Head Start Child's Social Competence at the end of the Head Start year, and at the End of Kindergarten

With respect to one important aspect of the Head Start child's social competence -mothers' reports of their Head Start children's cooperativeness at home -- more

\footnotetext{
47 The same analyses performed on a slightly different sample (Head Start children of mothers followed up in the telephone interview) found, after controlling for gender and pre-Head Start school-readiness, that greater attendance at Workshops and Policy Meetings was statistically significantly associated with better adaptation to the classroom, and that more Undifferentiated Involvement tended towards a statistically significant positive association.
} 
Undifferentiated Involvement was statistically significantly associated with mother's report of her child's greater cooperativeness at the end of the Head Start year. Volunteering was marginally statistically significantly associated with more cooperativeness, and more Workshops/Policy Meetings tended towards a statistically significant association. These findings are encouraging because they suggest that: 1) the effects of parent involvement in Head Start may benefit both the parent and the child -- having a more cooperative child may result in positive consequences for both; and 2) the benefits of Head Start may extend to settings other than Head Start, i.e., the home, and to child outcomes other than cognitive outcomes.

The Head Start child's CPI school-readiness was affected by the combination of involvement and engagement in self-sufficiency promoting activities. Children of engaged parents demonstrated more school-readiness when their parents were medium, but not highly involved. In contrast, children of not engaged parents demonstrated more schoolreadiness when their parents were highly involved. These results are suggestive that the total amount of activity the parent is engaged in, may affect the parent, and through the parent, the Head Start child.

For this sample of Dominican mothers, parent involvement did not have a clear effect on parents' reports of the Head Start child's disruptiveness at home and disruptiveness and cooperativeness were not seen as mutually exclusive. Because the measure also taps assertive behavior, the mothers may have perceived their children as normally assertive rather than disruptive. Consistent with this notion, the Head Start children's average score on the measure indicated a normal degree of demandingness/assertiveness for this age group, suggesting that a decrease due to involvement may not have benefitted the Head Start child.

Parent involvement in Head Start was not directly related to teacher ratings of the Head Start child's adaptation to the kindergarten classroom, nor to teacher ratings of the child's kindergarten and anticipated future performance. The zero-order correlations between the involvement measures and the outcomes were accounted for by differences in the child's pre-Head Start CPI school readiness scores. Because the hypothesized direct 
relationship was not found in this study, future research should concentrate on the development of a causal model which will predict the Head Start child's kindergarten competence and adaptation by identifying direct and indirect influences on the outcome. For example, since parent involvement in Head Start was shown in the present study to influence educationally relevant outcomes such as parental help with school-readiness skills, it is possible that variables such as these may indirectly mediate the effects of involvement in Head Start on elementary school performance.

\section{Parent Involvement and Older Sibling Outcomes}

- Is parent participation in Head Start positively related to the social competence of an older sibling of the Head Start child?

Forty-seven older siblings of the Head Start child were followed up as part of the telephone interview conducted one year after Head Start. The siblings were rated by their parents on the three competence subscales of the CBCL: Social, School, and Activities. An additional CBCL score, the Total Competence score, was created by summing the three subscales. The CBCL questionnaire also included data on the siblings' retention in grade, and placement in a special class. Thus, six variables were assessed.

For the sibling subsample as a whole, there were no changes from pretest to followup in Activities or Social CBCL scores (see Table 11). However, older siblings' Total Competence and School Competence, as rated by the parents, tended towards statistically significant decreases from the beginning of the Head Start year $[\mathrm{t}(35)=1.77, \mathrm{p}<.10$; and $t(36)=1.81, p<.10$, respectively]. For the sample as a whole, from pretest to the follow-up, there was no statistically significant change in number of siblings retained in grade or in special classes. At pretest, five older siblings were reported as having repeated a grade. At follow-up, no additional retentions were reported. At pretest, five of the 47 older siblings were reported to be in a special education class. At follow-up, two of these five were still in such a class along with five other siblings.

In order to test the hypothesis of a positive association between parent involvement in Head Start and older siblings' CBCL scores, partial correlations were computed between the three involvement variables and the four $\mathrm{CBCL}$ variables, controlling for the pretest (see Table 12). Because Volunteering tended towards a statistically significant association 
with Total Competence, the relationship was subjected to regression analysis which controlled for demographic factors related to the outcome as well as the pretest. As the other relationships did not approach statistical significance, analyses were conducted to explore possible complex relationships.

\section{Association of Parent Involvement with the Older Sibling's CBCL Total Competence One Year After Head Start}

As the partial correlation between Volunteering and total CBCL competence tended towards statistical significance, a regression was performed controlling for the pretest and two demographic variables -- sibling gender (boys tended to have higher overall competence scores than girls, $\mathrm{r}=.26, \mathrm{p}<.10$ ); and number of other children in the home (homes with less children tended to have older siblings with greater social competence, $r=-.25, \mathrm{p}<.10$ ). In support of the hypothesis, after controlling for demographic variables and the pretest, more Volunteering showed a trend toward a statistically significant association with higher CBCL Total Competence ( $\mathrm{r}$ square change $=.075, \mathrm{p}<.10$ ).

\section{Association of Parent Involvement with the Older Sibling's CBCL School Competence One Year After Head Start}

As the partial correlations between the three involvement variables and sibling's School Competence were not statistically significant, additional analyses were conducted to explore possible complex relationships. The first analysis addressed the possibility of a nonlinear relationship between Undifferentiated Involvement, measured categorically, and the sibling's school competence, but there was no evidence for a non-linear relationship.

The second analysis addressed the possibility that controlling for demographic, contextual, and personal variables which were related to the outcome would increase the precision of the analysis, revealing a positive relationship between involvement and siblings' school competence. This analysis was not statistically significant.

The test of the interaction of engagement in self-sufficiency promoting activities and involvement was not performed due to small sample size. 
As there was no evidence of a linear relationship between parent involvement in Head Start and the sibling's Social Competence, additional analyses were conducted to explore possible complex relationships. The first analysis addressed the possibility of a nonlinear relationship between Undifferentiated Involvement, measured categorically, and the sibling's CBCL social competence. Although there was no evidence of a non-linear relationship, there was evidence of a positive relationship between Undifferentiated Involvement, measured categorically, and the outcome. An analysis of covariance on posttest scores controlling for pretest scores with Undifferentiated Involvement as the independent variable tended towards statistical significance $[(F(2,40)=2.88, \mathrm{p}<.10]$. Siblings of parents in the low involved group $[\mathrm{F}(1,41)=5.77, \mathrm{p}<.02]$, but not in the medium or high involved groups, tended towards a statistically significant decrease in CBCL social competence.

The second analysis addressed the possibility that controlling for demographic, contextual, and personal variables which were related to the outcome would increase the precision of the analysis, revealing a positive relationship between involvement and siblings' social competence. This analysis was not statistically significant.

The test of the interaction of engagement in self-sufficiency promoting activities and involvement was not performed due to small sample size.

\section{Association of Parent Involvement with the Older Sibling's CBCL Activities Competence One Year After Head Start}

As there was no evidence for a linear relationship between involvement and the sibling's Activities competence, additional analyses were conducted that explored possible complex relationships. These analyses were not statistically significant. Due to small sample size, the analysis exploring the interaction of engagement in self-sufficiency promoting activities and involvement was not conducted. 
Association of Parent Involvement with the Older Siblings' Retention in Grade and Placement in a Special Class

Retention in Grade. Because no siblings were reported to have been retained in grade during the two-year study period, there was no variability with which to test the hypothesis.

Placement in Special Education Class. There was no evidence of a statistically significant relationship between involvement and placement in a special class, nor of a relationship between initial placement and placement two years later at follow-up. However, because of small sample size, these results are not conclusive.

Summary and Discussion of the Association of Parent Involvement with Older Sibling Outcomes

Despite the small sample size, the results revealed tentative evidence of a positive relationship between involvement and older sibling outcomes. After controlling for demographic variables and the pretest, Volunteering tended toward a statistically significant association with greater total social competence as measured by the CBCL. In addition, the analysis with a categorical measure of Undifferentiated Involvement revealed a trend towards a statistically significant association of involvement with CBCL social competence: In the former, more Volunteering was linked with more overall social competence. In the latter, more Undifferentiated Involvement prevented a decrease in sibling social competence. Thus, despite low power due to small sample size, parent's involvement in Head Start was shown to have benefits for others in the family, i.e., the older sibling, as well as for parents and the Head Start child (see. sections above). The results also indicate that the benefits of participation in Head Start last beyond the end of the program.

\section{Utilization of Social Services: Descriptives and Predictors}

\section{Description of Parents' Utilization of Social Services}

None of the other three types of involvement (Undifferentiated Involvement, Volunteering, Workshops/Policy) was correlated with Utilization of Social Services, supporting our notion that Social Services is qualitatively different from the other activity types, and thus, should be analyzed separately. The mean number of hours of social service 
contacts was 4.7 per parent, with the scores ranging from zero to 18 hours for the Head Start year. A square root transformation was applied to the Social Services scores in order to normalize the distribution.

The average number of hours Social Services staff spent with each parent over the Head Start year was low, despite the consensus of the Head Start Research Group that staff and parents interacted constantly around social service needs and goals. The low number of hours spent with individual families may be related to a current policy concern in Head Start regarding the appropriate number of families that should be assigned to each Social Services staff. Collins (1993) suggested that 30 to 50 families constitutes a reasonable caseload, depending on the severity of family life circumstance; while the Social Services Task Force of the Silver Ribbon Panel (National Head Start Association, 1990) recommended a family/Social Services staff ratio of 35:1. However, in focus groups on parent involvement held by the ACYF in 1995 as part of their deliberations on revising the Head Start Performance Standards, concerns were voiced regarding the effectiveness of the Social Services component in light of caseloads of 85-95 families per worker. In Agency One, each Social Services staff worked with an average of 72 families. Thus, while 4.7 hours per year may appear to be low for each family, it may be the maximum amount the staff can handle given their other responsibilities such as running workshops and policy meetings, assisting in the classroom, and completing paper work. Another reason for the low number of social services hours reported is that perhaps some social services contacts are not recorded by staff -- informal contacts, for example. If this were the case, parents would be receiving more social services than our data indicate. However, unreported data -- unless equally distributed among all parents -- would change the rank order of our Utilization of

Social Services variable. Nonetheless, since these data are the first to specifically assess Utilization of Social Services, we feel it is an important first step in exploring this phenomenon.

\section{- Predictors of Utilization of Social Services}

As with the other parent involvement variables, we were interested in learning which personal, demographic, and contextual variables correlated with parental Utilization of 
Social Services. To that end, we computed zero order correlations between the $38^{48}$ predictor variables (see Table 1) and parental utilization of Social Services. Ten of the 38 $(26 \%)$ predictor variables were associated with the extent of the mother's Utilization of Social Services: 1 ) older mothers $(\mathrm{r}=.17, \mathrm{p}<.05)$, and 2$)$ those with less education $(\mathrm{r}=-.22$, $\mathrm{p}<.01)$ utilized social services statistically significantly more often. 3 ) Those mothers who were stricter $(r=.17, p<.05)$ with their children; 4$)$ those who helped their child learn more school readiness skills $(\mathrm{r}=.19, \mathrm{p}<.05)$; and 5) those who had an older Head Start child $(\mathrm{r}=.17, \mathrm{p}<.05)$ or 6$)$ a parent with a Head Start child with more health problems since birth $(\mathrm{r}=.17, \mathrm{p}<.05)$ also spent statistically significantly more hours utilizing social services. 7$)$ Parents who talked more frequently with their Head Start child about school $(r=.13, p<.10)$ tended to have statistically significantly more contact with Social Services staff. Three sibling variables also were associated with more social service contacts: 1) Having an "older" older sibling ( $\mathrm{r}=.30, \mathrm{p}<.05)$; or 2$)$ a sibling with poorer school performance $(\mathrm{r}=-.23$, $\mathrm{p}<.10$ ); and 3 ) having a sibling with greater social competence (measures extent of and quality of peer and family relationships, as well as participation in organizations in the community) $(\mathrm{r}=.25, \mathrm{p}<.05)$. The direction of the relationship between sibling's social competence and Utilization of Social Services is consistent with anecdotal data from parents and staff. ${ }^{49}$ Perhaps parents were especially concerned about the safety of their older, socially competent children participating in community organizations in light of the physical dangers and unwanted social influences of their community. These concerns may have been shared with social services staff.

It was noteworthy that the psychological well-being variables -- depression, personal control, self-esteem -- were not related to social services utilization. Rather, concerns relating to the functioning of the children predicted this type of involvement.

\footnotetext{
48 This includes all variables in Table 1 except ethnicity, engagement in self-sufficiency promoting activities and parent's need for greater self-sufficiency reported at pretest. Ethnicity was excluded because $99 \%$ of the Agency One sample was Dominican. The other two variables were analyzed separately because of their theoretical importance.

${ }^{49}$ Personal communications from Agency One and Agency Two Head Start Social Service Coordinators describing their ongoing dialogues with Head Start parents of teenagers (June, 1993). Parents reported attempting to protect their older, socially competent children from the physical dangers and unwanted social influences of their community by accompanying them to sporting events, clubs, and other community activities.
} 
In order to determine the extent to which these predictors jointly explained variability in Utilization of Social Services, a stepwise multiple regression analysis was conducted. The three sibling variables were not included in this analysis because sibling data were not available for 86 of the 172 posttested families $(\mathrm{N}=86)$ without an older sibling, leaving seven predictors in the analysis. With all seven variables entered, the overall regression model was statistically significant (final Multiple $\mathrm{R}=.37, \mathrm{~F}=3.40, \mathrm{p}<.002$ ). The percent of variance accounted for jointly by the seven variables was $9.9 \%$. Mother's education, the health of the Head Start child since birth, and the extent of parent's helping the child learn school readiness skills all contributed statistically significant unique variance to the prediction of Social Services Utilization. Thus, mothers who utilized Social Services the most had less education, had children with more health problems from birth, and reported spending more time helping their child prepare for school.

As with the other types of involvement, we examined the relationship between two additional variables -- self-sufficiency needs and engagement in self-sufficiency promoting activities -- and extent of Utilization of Social Services. Only families whose Head Start child was beginning his or her first year in Head Start reported whether or not they had self-sufficiency related needs. The correlation between this variable and Utilization of Social Services was $\mathrm{r}=.18, \mathrm{p}<.05$, indicating that parents with these needs spent more hours with social services staff. In this subsample, self-sufficiency needs accounted for approximately four percent of additional variability in Social Services.

The relationship between engagement in self-sufficiency promoting activities during the Head Start year and Utilization of Social Services was not statistically significant. Thus, Social Services was the only type of involvement not associated with parents' self-sufficiency promoting activities.

\section{Utilization of Social Services and Parent, Head Start Child and Sibling Outcomes}

As with the other types of involvement, partial correlations were computed between Utilization of Social Services and the outcome variables, controlling for pretest differences. 
The outcome domains examined were: the parent-child relationship; the home learning environment; parents' well-being; engagement in self-sufficiency activities one year following Head Start; the Head Start child's social competence and school readiness; and sibling engagement in community organizations and activities, social competence, school performance, grade retention, and placement in special education. Of the partial correlations computed, only one outcome domain was affected by parents' use of Social Services: Similar to the relationships found between personal control and Volunteering and Undifferentiated Involvement (see previous section), contrary to expectation, greater Utilization of Social Services tended to be significantly associated with lower feelings of personal control $(\mathrm{r}=-.14, \mathrm{p}<.10)$.

There are several possible reasons for the lack of positive relationships between the Utilization of Social Services and our outcome variables. First, the data on the predictors of Utilization of Social Services indicates that some of the reasons parents sought Social Services -- and hence were variables most likely to be affected by it -- were not assessed as outcome variables included in the present study. For example, one of the predictors of Utilization of Social Services was having a Head Start child with more health problems since birth; yet we did not include a health domain among our outcomes. Thus, a different set of outcome variables may have revealed positive effects of utilization of social services.

Further, some predictors of social services were issues not addressed in the actual parent-staff contacts. For example, although aspects of parents' provision of the home learning environment predicted Utilization of Social Services, and may indicate parents' positive interest in and motivation to help their child, the scope of Social Service staff's job does not include child-related educational services in their one-on-one contacts with the parent (USDHHS, 1992). Other more pressing problems such as children's health, mother's educational future, and sibling's functioning are the purview of Social Services. Issues dealing with school-readiness are more appropriately addressed by the Volunteering and Workshops components of the program (see results reported earlier). Therefore, in order to better evaluate the effectiveness of the social services component of Head Start, future research should take into account the nature of the issues dealt with in each parent's social services contact and assess improvement in outcomes related to those issues. 
Second, as stated earlier, parents may have received too few in-depth Social Services contacts to have the desired changes occur. Similarly, some of the outcome measures themselves may not be sensitive enough to detect the small changes that may have occurred in the parent. An example comes from the possible relationship between parents' use of Social Services and parents' engagement in self-sufficiency activities such as job training, education and/or employment during the year following Head Start. There was no association of Utilization of Social Services with parents' engagement in self-sufficiency activities despite the fact that Social Services addresses educational and self-sufficiency issues. This lack of findings may be due to limitations in the nature of the data available in the present study. Our data represents large changes in parental status, and may not be sensitive to smaller improvements in the self-sufficiency of Head Start parents. Herr and her colleagues (Herr, Halpern, \& Majeske, 1995) have developed a taxonomy of job-related skills and their positions relative to each other on a "ladder" leading to self-sufficiency. In this framework, parent involvement activities in Head Start are matched with the job-skills they teach. For example, Social Services staff provide resources related to job training and often help parents make appointments for interviews or for enrolling in educational programs. These kinds of activities could serve as incremental steps towards a parent's achieving self-sufficiency. If data on these smaller steps had been available, they may have demonstrated a positive association of parents' use of Social Services on these outcomes. A content analysis of the issues discussed and the recommendations made by social services staff during their interactions with the parents might shed light on this issue, and is recommended for future research.

Third, the relationship between Utilization of Social Services and outcomes often appeared to be complex. For example, Utilization of Social Services tended towards a statistically significant association with personal control $(r=-.14, p<.10)$. However, it was in the direction opposite to prediction: Greater Utilization of Social Services was associated with lower feelings of personal control at posttest. Post hoc analyses did not provide a clear picture of the processes at work. Thus, further research is needed to identify the variables 
and/or processes mediating the relationships between different types of involvement and changes in personal control. ${ }^{50}$

Finally, the present study took only the first step in developing an understanding of the impact of Social Services on the family by conceptualizing Social Services as amount of time spent by the parent with Social Services staff. However, this metric does not reflect more complex processes such as the quality and strength of the relationship which the family service worker develops with the Head Start parent. In addition to examining the nature of the issues dealt with in the Social Service contacts, a crucial next step will be to gain an understanding of whether and how this ongoing interpersonal relationship produces the benefits that so many believe are a result of participation in the Social Services component of the Head Start experience.

\section{Summary and Conclusions}

The inclusion of the contact of parents with Social Services/Parent Involvement staff as another type of parent involvement seems to be a critical part of the Head Start experience for families by all reports (Collins, 1993; Melon, 1995; Parker, Piotrkowski, \& Peay, 1987; Powell, 1989; Replogle, 1995). However, up to the present and including this study, few clear, empirical, causal relationships have been documented of involvement in the Social Services/Parent Involvement component of Head Start and positive changes in parents and their children after Head Start. It is one of the most elusive phenomena of Head Start's story. It is unclear why the empirical data are not consistent with the anecdotal accounts of the positive impact of social services on parents. This study suggests several limitations on our ability to fully assess how this type of parent involvement is linked to parent outcomes.

The first issue involves the caseload of Social Services/Parent Involvement staff and the need to keep accurate records. Social Services/Parent Involvement staff might need to

\footnotetext{
${ }^{50}$ Third variables related to both the outcome and to social services utilization may result in a spurious negative correlation between social services utilization and this outcome. One such scenario might result from parents' experiencing stress from adverse life events during the Head Start year. The experience of lack of control over such life events may. result in both lowered feelings of personal control and in greater utilization of social services to deal with the consequences of the life events.
} 
document every single, however small, interaction between themselves and the parents, almost like the "process notes" of a Social Worker, in order to capture more of the nature of the evolving relationship between staff and parents. Additionally, the time and effort expended by the Social Services staff on behalf of parents -- but not in their presence -- may also need to be documented in order to adequately measure the extent of Utilization of Social Services. This information could then be coded, and a more detailed measure could be developed than the one used in the present study. However, with a family caseload averaging 85 in Head Starts nationwide, this route at present is an impossibility unless a small, subsample of Head Start parents were chosen for this experiment.

The Peer Group strategy developed for the Parent Involvement Project (see Parker, Piotrkowski, Young, Clark, Peay, Flynn, \& Baker, 1996) attempted to move Social Services/Parent Involvement staff in that direction. Qualitative data from that intervention revealed that offering more intensive social services to a targeted, small group of parents, giving peer support to that staff, building their professional skills, and keeping better records for assessing impact, resulted in perceived changes in some important parental outcomes, including personal control, movement toward self-sufficiency, and improved parent-child relationships.

It is especially important to provide adequate training for social services staff because these staff are primarily recent Head Start parents with many of the same major life stresses as the current Head Start parents they must help. Their task is made even more difficult by the complex nature of the network of available community and government services which they utilize to help Head Start parents.

The second issue involves having more sensitive measures for detecting small changes in the desired outcomes and measures of outcomes reflecting the issues that are dealt with by Social Services staff and parents (e.g., child health). It might be necessary to develop a qualitative approach to more fully understand how and why social services contacts appear so influential for some parents by anecdotal account.

The third issue, and probably the most important, involves the exploration of potential theoretical models of what outcomes are likely to be affected by Social Services/Parent Involvement staff. There may be mediating variables between parent-staff 
contacts and parent and child outcomes that have not yet been explored. One early attempt in that direction was the dissertation work of Parker, Piotrkowski, and Peay (1987). In that study it was theorized that Head Start served as an institutional social support for parents and that support was part of the parent involvement relationship that was then associated with parents' psychological well-being and attitudes in the parent-child relationship. This early work needs to be translated into testable research questions that focus on unpacking this "black box." The Peer Group strategy conducted in the 1992-93 Head Start year, described in Parker, Piotrkowski, Young, Clark, Peay, Flynn, and Baker (1996), also attempted to address this intangible quality in the parent-staff relationship that seems to make a difference. Barnard (1995) suggested that mother's initial and changing attachment style over the course of the Head Start year will be related to ongoing family-staff relationships, family functioning, and the development of the identified child.

As a national early intervention model program that is over 30 years old, Head Start has been taking a critical look at the gaps in understanding of how and why the program seems to be so successful with many parents. A recent evaluation of the Head Start Performance Standards revealed that the Mental Health Component is the least understood and the least developed. Ironically, the Social Services/Parent Involvement staff have traditionally been the staff most closely involved with parents, and have had to help parents deal with their increasingly difficult life circumstances due to the ever-growing negative impact of poverty. Creative research in this area continues to be important and necessary for a clearer understanding of Head Start's role in helping families.

\section{RESULTS: AGENCY TWO}

\section{Demographic, Contextual, and Personal Characteristics of the Sample at Pretest}

The 103 pretested families at Agency Two were English-speaking and American-born and educated for the most part, though they were ethnically diverse. Highlights of the specific demographic and contextual factors that characterized these mothers, Head Start children, and their families are presented below (also see Table 2). 


\section{Mothers}

Mothers ranged in age from 18 to 54 (average age was 31 years). Only fourteen percent gave birth to their Head Start child before the age of 20. Most of the mothers were native United States citizens (82\%) and English-speaking (90\%). Their diverse ethnic backgrounds included: African-American (60\%); Hispanic (18\%); and Euro-American $(18 \%)$. Most of the mothers were educated in the United States. Thirty-six percent were high school drop-outs; $50 \%$ completed high school or its equivalent; and the remainder $(14 \%)$ received some training or education beyond high school. Almost four fifths of a subsample of 52 mothers for whom data were available ${ }^{51}$ reported needing additional education, job training, or employment in order to attain economic self-sufficiency.

\section{Head Start Children}

Of the 103 pretested Head Start children, 54\% were girls. They ranged in age from 2 years 8 months to 4 years 7 months. Most (64\%) were enrolled in Head Start for the first time, but $36 \%$ were returning for a second year.

\section{$\underline{\text { Families }}$}

Approximately half of the families at Agency Two were headed by a single mother. Another $36 \%$ lived with one other adult who was either a relative, friend, or partner.

For $16 \%$ of the families at Agency Two the Head Start child was an only child. Thirty-three percent of the families had one other child, but most commonly for this group, $51 \%$ of the families had two or more children in addition to the Head Start child. For nearly half $(49 \%)$ of all of the families, at least one of the other children was younger than six years. In $20 \%$ of the families at Agency Two, another pregnancy or birth was reported during the Head Start year.

Families' economic and physical resources were clearly inadequate. At intake, families' total income were under $\$ 10,000$ per year, and for $99 \%$ of these families, some or all of this was derived from AFDC benefits. At intake, $40 \%$ of families reported housing,

\footnotetext{
${ }^{51}$ Data were available on the self-sufficiency needs of families of children starting their first year of Head Start.
} 
space, or repair problems and/or inadequate utilities. Sixty-three percent of the mothers felt that their neighborhood was safe and most (87\%) intended to remain there the following year.

\section{Changes in Sample Size Over the Course of the Study}

In order to determine the population of families to whom our results would generalize, a set of analyses was conducted comparing the families who finished the posttest to the families who did not. At Agency Two, 151 families were eligible to participate in the study, 113 of them (74\%) signed consent forms to participate, and 103 families completed the pretest. Eighty-two of these 103 families also completed the posttest, representing an attrition rate of $20 \%$. T-tests were performed comparing the posttested and non-posttested families on the demographic, contextual, and personal variables listed in Table 3.

Of the $24^{52} \mathrm{t}$-tests performed comparing those who completed the posttest and those who did not, three were statistically significant and one tended towards statistical significance. One of the differences involved family characteristics: posttested parents reported more social support $[\mathrm{t}(101)=-3.34, \mathrm{p}<.001]$ than parents who were not posttested. With respect to parenting, posttested parents had lower expectations for their Head Start child's school performance [ $\mathrm{t}(23.4)=-3.20, \mathrm{p}<.004]$; and they showed a tendency towards less encouragement of their child's independence $[t(89)=1.71, p<.10]$. With respect to the Head Start child's characteristics, there was a statistically significantly greater percentage of girls among the posttested parents [chi $\mathrm{sq}(102)=8.12, \mathrm{p}<.01]$. Thus, the mothers who stayed in Head Start had less favorable scores at pretest on some childrearing-related variablesbut perceived more social support that may have enabled them to remain in Head Start over the full program year.

\footnotetext{
52 The sibling variables were excluded because there were not enough non-posttested families with siblings to constitute a viable group for comparison.
} 


\section{Description of Parents' Involvement in Head Start}

\section{Undifferentiated Involvement}

While virtually all of the parents (97\%) participated in some Head Start activities, there was considerable variation from parent to parent (see Table 13). Parents averaged approximately 54 hours over the course of the Head Start year, ranging from zero to 419 . Because the Head Start year was ten months, this was equivalent to a mean of approximately 5.4 hours per month. A logarithmic transformation was applied to the scores to normalize the distribution for subsequent analyses.

\section{Involvement by Type of Activity}

Volunteering in the classroom, office, kitchen, and on trips accounted for approximately $49 \%$ of total participation (see Table 14), or an average of 26.4 hours over

the Head Start year. Parents' volunteering ranged from zero to 299 hours, with $21 \%$ not volunteering at all.

Workshops/Policy Meetings accounted for approximately 51\% of total participation, averaging approximately 27 hours over the Head Start year, and ranging from zero to 255 hours. Fourteen percent of the parents did not participate in any workshops or policy activities over the course of the year. Logarithmic transformations were applied to both activity types in order to normalize the distributions.

\section{Intercorrelations among the (Untransformed) Parent Involvement Variables}

Both Volunteering and Workshops/Policy Meetings were highly correlated with Undifferentiated Involvement $(r=.89$ and $r=.92$, p's <.001, respectively; also see Table 14). Volunteering and Workshops/Policy Meetings also were statistically significantly correlated with each other $(r=.63, p<.001)$.

In summary, in this sample virtually all mothers participated in some Head Start activities. Although the participation of mothers varied widely, average number of hours of 
participation was only five hours per month. ${ }^{53}$ Volunteering accounted for approximately half of the total involvement hours; however, one-fifth of the mothers did not volunteer at all. At this agency, attending workshops and policy meetings was clearly an important part of the Head Start experience for families since attendance at them accounted for half of the total involvement hours.

\section{Predictors of Parent Involvement: Barriers and Facilitators}

\section{- What are the demographic, contextual, and personal variables related to parent participation in Head Start?}

Objective 1, to identify through a longitudinal study, the "predictor" variables associated with parents' participation in Head Start, was addressed in analyses conducted on the families who completed the posttest $(n=82)$. Zero-order correlations were computed between each involvement variable and $41^{54}$ demographic, contextual, and personal variables relating to characteristics of the mother, Head Start child, and older sibling at pretest (see Table 1). The number of statistically significant predictors and trends was below that expected by chance for each of the three involvement variables. Because the number of statistically significant findings was below chance, these findings were not considered reliable. 55 This may be due in part, perhaps, to the large number of possible predictors that were examined in an attempt to be exhaustive.

${ }^{53}$ Compared to Agency One parents, Agency Two parents volunteered half as many hours, and spent two-thirds as many hours, in workshops and policy meetings.

54 This.includes all of the variables in Table 1 except parents' need for self-sufficiency reported at pretest and parents' engagement in self-sufficiency promoting activities during the Head Start year. These two variables were analyzed separately because of their theoretical importance.

55 Because two of the relationships found at Agency Two were also found at Agency One, these relationships may represent reliable findings: 1) Not being pregnant was associated with more Undifferentiated Involvement ( $r=-.29$, $p<.05$ ), less Volunteering $(r=-.33, p<.05)$, and less Workshops/Policy Meetings $(r=-.23, p<.05)$; and 2$)$ having an older sibling with better CBCL school competence was associated with more Undifferentiated Involvement $(r=.37, p<.05)$ and more Volunteering $(\mathrm{r}=.43) .2)$. The other variable associated with more Undifferentiated Involvement in this sample was helping the Head Start child learn more school readiness skills $(r=.19)$. The other variables associated with more Volunteering in this sample were: 1) having a Head Start child with less health problems ( $r=-.23)$; and 2$)$ helping the Head Start child leam more school readiness skills. The other variables associated with more Workshops/Policy Meetings in this sample were: 1) talking more with one's Head Start child about school ( $r=.23)$; and 2) letting one's Head Start child help with more household tasks $(r=.19)$. These other variables may not represent reliable findings. 


\section{Self-Sufficiency Needs, Engagement in Self-Sufficiency Promoting Activities During the} Head Start Year and Extent of Parent Involvement

Two other variables which might affect the extent of parents' involvement also were explored: whether parents reported self-sufficiency needs at pretest and their pursuit of self-sufficiency activities outside Head Start over the course of the Head Start year.

Over $80 \%$ of the mothers for whom data were available ${ }^{56}$ reported needing one or more of the following: paid employment, additional education, or job-training. However, the correlations between this predictor and the three involvement variables were not statistically significant.

Approximately $48 \%$ of the full sample of posttested mothers reported that they were engaged in self-sufficiency promoting activities over the course of the Head Start year. However, the correlations between engagement and the three involvement variables were not statistically significant.

\section{Summary and Discussion of the Findings on the Predictors of Involvement}

Involvement at Agency Two was not well predicted by the demographic, contextual, and personal variables tested in this study as the number of variables predicting each type of involvement was lower than that expected by chance. ${ }^{57}$ Part of the reason for the low number of statistically significant relationships relative to the number of predictors examined may be that in our attempt to be exhaustive, we were overly inclusive of variables that may have a lower probability of being predictors.

It is not clear why self-sufficiency needs and engagement in self-sufficiency activities were not statistically significantly associated with extent of involvement, as they were in Agency One. Perhaps the lower involvement of the Agency Two mothers, compared with those of Agency One, conflicted less with their engagement in self-sufficiency promoting activities.

\footnotetext{
${ }^{56}$ Data were available for the subsample of posttested mothers $(\mathrm{N}=45)$ whose child was beginning his or her first year in Head Start.

${ }^{57}$ Nevertheless, in this sample of Agency Two mothers, pregnancy was associated with less participation. Because this result is also found in Agency One mothers, it may represent a true relationship rather than a spurious one.
} 
As in Agency One, the personal well-being variables (depression and personal control) did not predict parents' involvement. Contrary to expectation, depression was not associated with decreased activity, at least with respect to Head Start.

\section{Parent Involvement and Parent Outcomes}

- Is parent participation in Head Start positively related to effective parent-child relationships?

Parents were assessed on four aspects of the parent-child relationship at the beginning and at the end of the Head Start year: 1) aggravation; 2) encouragement of independence; 3) frequency of daily parenting hassles, and 4) parental strictness. At pretest, the parents reported moderately positive parent-child relations (see Table 15). For the sample as a whole, only parenting aggravation changed, marginally statistically significantly decreasing over the Head Start year $[t(78)=1.95, p<.055]$.

In order to test the hypothesis of a positive association between involvement and the parent-child relationship variables, partial correlations were computed between the three involvement variables and the four aspects of the parent-child relationship, controlling for pretest differences (see Table 16). The outcome statistically significantly related to involvement (aggravation) then was subjected to regression analysis which controlled for demographic factors related to the outcome as well as for the pretest. The results of this analysis are reported below. As the partial correlations between involvement and the other three parent-child outcomes (strictness, encouragement of independence, frequency of daily hassles) were not statistically significant in the expected direction, exploratory analyses were conducted. The additional analyses with respect to strictness were not statistically significant. The results of the additional analyses on encouragement of independence and frequency of daily hassles are reported below.

\section{Association of Parent Involvement with Parenting Aggravation}

As the partial correlation between Volunteering and aggravation was statistically significant, a regression was conducted which controlled for mother's age and years the parent was in Head Start, the two demographic factors related to the outcome [younger 
mothers $(r=-.21, \mathrm{p}<.10)$ and mothers in Head Start more than one year $(r=.28, \mathrm{p}<.05)$ experienced more posttest aggravation]. In support of the hypothesis, after controlling for relevant demographic factors and the pretest, more Volunteering was statistically significantly associated with less parenting aggravation [ $\mathrm{r}$ square change $=.033, \mathrm{p}<.05$ ].

\section{Association of Parent Involvement with Parental Encouragement of the Head Start Child's} Independence

As there was no evidence of a linear relationship between involvement and parental encouragement of the child's independence, additional analyses were performed to explore possible complex relationships. Although the first two analyses (non-linear and covariate regression analyses) were not statistically significant, there was evidence of an interaction between Undifferentiated Involvement, measured categorically, and engagement in selfsufficiency activities on parental encouragement of independence. An analysis of covariance of posttest means controlling for the pretest showed a trend towards statistical significance for the interaction $[\mathrm{F}(2,69)=2.44, \mathrm{p}<.10]$. An examination of the posttest means adjusted for the pretest (see Figure 3 ) indicated that amount of involvement had a differential effect on the unengaged parents: In the not engaged group, medium involved parents encouraged their child's independence less than low or high involved parents.

\section{Association of Parent Involvement with Frequency of Daily Parenting Hassles}

Two of the involvement variables -- Undifferentiated Involvement and Workshops/Policy Meetings -- were not significantly associated with frequency of daily parenting hassles. Although the partial correlation between the other activity type, Volunteering, and the outcome was statistically significant, it was in the direction opposite to expectation. Thus, at Agency Two, more Volunteering was statistically significantly correlated with more frequent parenting hassles $(\mathrm{r}=.25, \mathrm{p}<.05)$. The correlation of Undifferentiated Involvement and frequency of daily hassles, although not significant, was also in the opposite direction.

Post-hoc analyses (non-linear relationship, covariate regression analysis, interaction of involvement and engagement in self-sufficiency activities) were conducted with 
Undifferentiated Involvement as the independent variable. Although these analyses were not statistically significant, there was a statistically significant relationship between engagement in self-sufficiency promoting activities and frequency of daily hassles independent of involvement. Engaged parents reported statistically significantly more frequent daily parenting hassles both at the beginning and at the end of the Head Start year. Thus, in this sample of Agency Two mothers, being involved in many activities -engaged either outside of Head Start or volunteering in Head Start -- was associated with more perceived daily parenting hassles.

\section{Summary and Discussion of the Association of Parent Involvement with the Parent-Child} Relationship

Involvement had a statistically significant positive association with parenting aggravation, one aspect of the parent-child relationship measured in the present study. After controlling for demographic variables and the pretest, more Volunteering was statistically significantly associated with less parenting aggravation at the end of the Head Start year.

Interestingly, although more Volunteering was also associated with more perceived daily parenting hassles, these hassles did not result in more parenting aggravation. Thus, Volunteering seems to have had a positive impact on parents' perceived level of stress, but a negative impact on parent's perception of the child's behavior. From the data of the present study, we are unable to determine whether parenting hassles have in fact increased for parents who volunteered more, or whether parents perceive more hassles because they are busy.

There was tentative evidence for an interaction of Undifferentiated Involvement and engagement in self-sufficiency promoting activities on parental encouragement of the child's independence: Medium involved not engaged parents tended to encourage their Head Start child's independence less than the other five groups. The reason for this relationship was not clear. 
- Is parent participation in Head Start positively related to a positive home learning environment?

Three aspects of the home learning environment were assessed at the beginning and end of the Head Start year: 1) number of educationally relevant play materials provided by the parent for the home; 2) number of school readiness skills the parent helped her Head Start child learn; and 3) parents' expectations for the Head Start child's school performance. Parents had generally high expectations for their children's school success, helped their children learn almost all of the school-readiness skills included in the measure, and provided a moderate number of play materials in the home. For the sample as a whole, one of the three variables showed change over the Head Start year: number of play materials in the home increased significantly $[t(73)=-4.38, p<.001$, see Table 15]. Because parents already were helping their children learn almost all of the school-readiness skills, this variable was subject to possible ceiling effects.

Partial correlations were computed between the three involvement variables and the three aspects of the home learning environment controlling for pretest differences (see Table 16). The outcome with a statistically significant relationship with involvement (play materials) then was subjected to regression analysis which controlled for demographic factors related to the outcome as well as for the pretest. The results of the regression analysis are reported below. As the partial correlations for the other two outcomes (parents' helping their children learn school-readiness skills, parents' expectations) were not statistically significant, exploratory analyses were conducted on these outcomes. The exploratory analyses on the variable measuring parents' help with school-readiness skills were all not statistically significant, probably due to a ceiling effect. Analyses on parents' expectations are reported below.

Association of Parent Involvement with Number of Educationally Relevant Play Materials in the Home

As the partial correlation between Volunteering and play materials was statistically significant, a regression was conducted controlling for the pretest and three demographic 
variables associated with this outcome [having more education $(\mathrm{r}=.21, \mathrm{p}<.10)$ tended towards a statistically significant association; and having an older Head Start child $(r=.25$, $\mathrm{p}<.05)$, and one with a higher CPI school readiness score $(\mathrm{r}=.25, \mathrm{p}<.05)$ had statistically significant associations with more play materials in the home]. In support of the hypothesis, after controlling for the demographic variables and the pretest, more Volunteering was statistically significantly associated with the parent's providing more play materials in the home ( $\mathrm{r}$ square change $=.05, \mathrm{p}<.05$ ).

\section{Association of Parent Involvement with Parent's Expectations for the Head Start Child's School Success}

As there was no evidence of a linear relationship between involvement and the parents' expectations for the Head Start child's school success, additional analyses were conducted to explore possible complex relationships.

The first analysis revealed tentative evidence of a non-linear relationship between level of Undifferentiated Involvement, measured categorically, and parents' expectations for the child's school success. An analysis of covariance on parents' posttest expectations, adjusted for the pretest, tended towards statistical significance $[F(2,64)=2.41, p<.10]$. An examination of the posttest means adjusted for the pretest indicated that the medium involved parents tended to have higher expectations for their child's school success than both the low and high involved parents.

The other exploratory analyses (covariate regression analysis, interaction of involvement and engagement in self-sufficiency promoting activities) were not statistically significant.

Summary and Discussion of the Association of Parent Involvement with the Home Learning Environment

There was evidence that one type of parent involvement -- Volunteering -- was statistically significantly associated with parents' provision of educationally relevant play materials in the home, an important aspect of the home learning environment. Even after controlling for demographic factors and the pretest, more Volunteering was statistically 
significantly associated with more play materials in the home. Thus, parents' volunteering in Head Start resulted in benefits for the Head Start child and other siblings at home, giving them additional opportunities to develop motor and cognitive skills by the use of educationally relevant play materials.

There also was tentative evidence for a non-linear relationship between involvement and parents' expectations for the Head Start child's school success. Although all groups of parents had high expectations, the medium involved parents tended to have the highest expectations.

The lack of a relationship between involvement and parents' helping the Head Start child learn school-readiness skills might have been due to a ceiling effect: at the beginning of the Head Start year, parents on average were already helping their child learn almost all of the skills included in the measure.

- Is parent participation in Head Start positively related to parental well-being?

Parents were assessed at pretest and again at posttest on two aspects of well-being: 1) personal control/mastery, and 2) depression. At pretest, parents reported moderately positive feelings of personal control, but a level of depressive symptomatology close to the criterion for clinical depression. At the end of the Head Start year, parents reported statistically significant improvement on both aspects of well-being, although depressive symptomatology remained relatively high.

Partial correlations were computed between the three involvement variables and the two aspects of well-being controlling for pretest differences (see Table 16). As none of the partial correlations approached statistical significance, additional analyses were conducted to explore alternative complex relationships. The additional analyses with respect to depression were not statistically significant, whereas one of the post-hoc analyses on personal control was. There was tentative evidence of a relationship between engagement in self-sufficiency activities and feelings of personal control, independent of involvement. Engaged and non-engaged parents did not differ significantly in personal control at pretest, but did differ at the end of the year. Engaged parents increased statistically significantly 
over the Head Start year $[F(1,76)=6.72, \mathrm{p}<.02]$ and ended the year with greater feelings of personal control $[\mathrm{F}(1,76)=4.95, \mathrm{p}<.03] . .^{58}$

\section{Summary and Discussion of the Association of Involvement with Parental Well-Being}

The link between parent involvement and increased emotional well-being of Head Start parents found in previous work by the authors (Parker, Piotrkowski, \& Peay, 1987) was not replicated here. In the discussion section, we discuss possible reasons for this lack of replication.

\section{Parent Involvement and Head Start Child Outcomes}

- Is parent participation in Head Start positively related to the social

-... competence of the Head Start child?

Three aspects of the Head Start child's social competence were assessed at the beginning and end of the Head Start year: 1) compliance/cooperativeness at home; 2) disruptiveness/assertiveness at home; and 3) Cooperative Preschool Inventory (CPI) schoolreadiness. Only Head Start children's CPI score changed significantly from pretest to posttest (see Table 17), indicating increased school readiness at the end of the Head Start year $[t(75)=-10.76, p<.0001]$.

Partial correlations were computed between the three involvement variables and the three child outcomes (see Table 18). As none of the partial correlations between the involvement variables and the Head Start child outcomes were statistically significant, additional analyses (non-linear relationship with involvement, covariate regression analysis, interaction of involvement and engagement in self-sufficiency promoting activities) were conducted to explore possible complex relationships. However, none of the exploratory analyses was statistically significant.

\footnotetext{
${ }^{58}$ However, a two-way analysis of covariance controlling for pretest scores did not show a significant main effect for engagement.
} 


\section{DISCUSSION}

This discussion section is divided into four sections: (1) summary and interpretation of major findings; (2) additional methodological considerations;

(3) recommendations for future research; and (4) policy and programmatic implications and suggestions.

\section{Summary and Interpretation of Major Findings}

\section{Predictors of Parent Involvement}

The first objective addressed by the present research was to identify through a longitudinal study the "predictor" variables associated with parents' participation in Head Start. At Agency One, approximately one fourth of the 40 demographic, contextual, and personal variables measured at pretest predicted at least one of the three types of involvement (Overall, Volunteering, Workshops/Policy Meetings). These variables explained $10-20 \%$ of the variability in parents' involvement.

The following variables emerged as predictors ${ }^{59}$ of more than one type of involvement at Agency One:

- Parents who reported needing education, job training, or paid employment at the beginning of the Head Start year participated more overall $(p<.01)^{60}$, volunteered more $(p<.05)$, and spent more time in workshops and policy meetings $(p<.05)$.

- Parents who were pregnant during the Head Start year participated less overall $(p<.05)$, volunteered less $(p<.05)$, and spent less time in workshops and policy meetings $(p<.05)^{61}$.

- Parents who engaged in self-sufficiency promoting activities during the Head Start year participated less overall $(p<.01)$, volunteered less $(p<.05)$, and spent less time in workshops and policy meetings $(p<.01)$.

\footnotetext{
59 As the present study used more conservative two-tailed tests in evaluating the benefits of Head Start, trends are also included here because they represented meaningful effect sizes (Cohen, 1977).

${ }^{60}$ The significance tests are two-tailed.

61 Although the total number of significant predictors of Workshops/Policy Meetings was below that expected by chance, it is likely that this relationship is reliable because pregnancy was correlated with all of the three involvement variables at both Agencies One and Two.
} 
- Parents who reported less frequent parenting hassles participated more overall $(p<.05)$ and volunteered more $(p<.05)$.

- Parents who reported less intense parenting hassles participated more overall $(p<.10)$ and volunteered more $(p<.10)$.

The following variables predicted one type of involvement at Agency One:

- Parents with a younger Head Start child participated more overall $(p<.10)$.

- Parents whose child was beginning his or her first year in Head Start participated more overall $(p<.10)$.

- Parents whose Head Start child experienced fewer birth difficulties volunteered more $(p<.10)$.

- Parents whose Head Start child experienced fewer health problems volunteered more $(p<.10)$.

- Parents who reported the older sibling to have greater school competence participated more overall $(p<.10)$.

At Agency Two, involvement was not well predicted by this set of variables because the number of variables associated with each type of involvement was below those expected by chance. A large number of potential predictors of involvement were explored in an attempt to be exhaustive, and this may have contributed to the low percentage of statistically significant findings. Nevertheless, two findings replicated those at Agency One: At Agency Two pregnancy during the Head Start year was associated with less participation overall, less volunteering, and less hours spent in workshops and policy meetings; and parents who reported that the older sibling had better school performance participated more overall and volunteered more.

The present study examined a large range of predictors related to parents' psychological characteristics, parenting attitudes, the home learning environment, the Head Start child and older sibling characteristics, and family demographic variables. The total amount of variability accounted for by this set of predictors was modest, suggesting that the next step in understanding which variables predict parent involvement in Head Start requires the development of a theoretical model specifying the most important factors influencing the extent of parents' participation. Additional variables which were not 
examined in the present study include requests from Head Start staff; characteristics of the Head Start program, educational needs of the Head Start child, the skills and preferences of the parents, and significant events impinging on the family during the Head Start year (e.g., death in the family, separation).

\section{Outcomes Associated with Parent Involvement}

The results of the present study provide modest support for the hypothesis that more parent involvement in Head Start is associated with improved outcomes for the Head Start parent, her Head Start child and an older sibling. The findings listed below represent positive linear relationships between one or more types of parent involvement and each outcome, controlling for important demographic factors and pretest scores.

Parent Involvement and the Parent-Child Relationship, Home Learning Environment, and the Social Competence of the Older Sibling

At Agency One, greater parent involvement was associated with an improved parentchild relationship, enhanced home learning environment, and parents' perception of greater social competence of the Head Start child and an older sibling as evidenced in the following seven outcomes:

- Greater parental encouragement of the Head Start child's independence $(p<.10)$

- Less frequently perceived parenting hassles $(p<.10)$

- Less parental strictness $(p<.06)$

- Greater number of school-readiness skills parent tries to teach her Head Start child $(p<.01)$

- Higher parental expectations for the Head Start.child's-long-term school success $(p<.10)$

- Parent's perception of her Head Start child as more cooperative at home $(p<.05)$

- Parent's report of the older sibling's greater overall social competence one year after Head Start $(p<.10)$ 
At Agency Two, greater parental involvement was linked with an improved parentchild relationship and enhanced home learning environment, as evidenced in the following outcomes: ${ }^{62}$

- Less parenting aggravation reported by parents $(p<.05)$

- Parental provision of more educationally relevant play materials in the home $(p<.05)$

The above nine findings showing positive associations between parent involvement and measures of the parent-child relationship, the home learning environment, parents' perceptions of their Head Start child's and older sibling's social competence provide evidence -- albeit modest -- of the effectiveness of Head Start resulting in benefits for the parent, Head Start child, and an older sibling through interventions with the parents (Collins, 1993; Parker, Piotrkowski, Horn, \& Greene, 1995; Zigler \& Valentine, 1979). First, these results provide modest evidence of the effectiveness of Head Start in helping mothers to be better parents, and experience less parenting stress and an enhanced parent-child relationship. Second, in addition to the already established direct benefits of Head Start for children (McKey et al., 1985; USDHHS, 1996), this study suggests potential indirect benefits for children as their parents' childrearing attitudes and ability to provide a more enriched home learning environment become more positive. These findings point to the success of Head Start in meeting a major programmatic goal to improve the lives of at-risk children living in poverty.

The present study was the first to gather data exploring the association of parent involvement with older sibling outcomes. Although the results were suggestive that greater volunteering was positively associated with the sibling's enhanced social competence, this study did not provide a full test of the relationship between involvement and sibling outcomes because the sample size was small and a full range of outcomes was not assessed. Another test of the hypothesis in future research would be to assess the benefits of parent

\footnotetext{
${ }^{62}$ At both agencies, greater parent involvement was associated with an improved parent-child relationship and home learning environment. However, the smaller number of positive findings for Agency Two may result from lower levels of parent involvement overall, a smaller sample size, or pretest differences in the amount of stress the parents experienced (Agency Two parents reported more stressors and higher levels of perceived stress). However, because the two agencies differed in several ways, firm conclusions cannot be drawn from the present data about the reasons for the somewhat different patterns of results. See discussion of Design Limitations to follow.
} 
involvement on a sufficiently large sample, some of whom are younger siblings, as they may also be more likely to benefit from change within the family.

\section{Parent Involvement and Parents' Economic Self-Sufficiency and Parental Well-Being}

The results of the present study also provide tentative support for the hypothesis that parent involvement in Head Start is positively associated with outcomes related to the selfsufficiency of the family: ${ }^{63}$

- Greater involvement in Head Start was associated with decreased likelihood of being pregnant during the year following Head Start $(p<.05)$.

- Greater involvement in Head Start was associated with increased likelihood of having paid employment $(p<.10)$.

The above two findings provide support for Head Start's effectiveness as a twogeneration program in improving the self-sufficiency of parents through their involvement. Greater parent involvement was associated with greater likelihood of employment and reduced likelihood of being pregnant during the year following Head Start. The financial benefit of paid employment, and the absence of the stress associated with pregnancy may benefit the children as well as the parents, resulting in "multiple payoffs" for the entire family from the parent component of the Head Start program. The finding that more overall involvement and more volunteering are associated with a greater likelihood of not being pregnant during the year following Head Start is particularly important in the current harsh economic climate. In a time of shrinking social resources and difficulty obtaining essential benefits such as health insurance from low wage jobs (Piotrkowski \& Kessler-Sklar, 1996), not being pregnant removes one source of physical, emotional, and financial stress for the mother as well as the entire family. A mother who is not pregnant may have more psychological and physical resources available to care for her family, and to deal with other life challenges, e.g., marriage, parenting, employment, education, and other life stresses.

The findings with respect to parents' engagement in self-sufficiency promoting activities are very encouraging because they were obtained despite the limited sample size, the molar-level measures of self-sufficiency promoting activities, and the institution of the

\footnotetext{
${ }^{63}$ This hypothesis was tested at Agency One only.
} 
welfare-to-work law aiming to enhance the self-sufficiency of all Head Start parents receiving public assistance regardless of their level of involvement in Head Start.

Thus, overall, the results provide tentative evidence that Head Start benefits parents and children in several ways, through improvement in the parent-child relationship, an enhanced home learning environment, and increased self-sufficiency.

Contrary to previous research (e.g, Parker, Piotrkowski, \& Peay, 1987), in the present study parent involvement in Head Start was not associated with increased parental psychological well-being, notably depression and personal control/mastery. Three factors may in part account for this: differences in the conceptualization and measurement of parent involvement; ${ }^{64}$ lower levels of participation; ${ }^{65}$ and changes in the larger society negatively influencing the life circumstances of parents in the 1990's. This third explanation is of particular importance to policy makers. The results of post-hoc tests at Agency One suggested that parents may be overloaded by the additional burden of engaging in selfsufficiency promoting activities while simultaneously raising young children and participating in Head Start. ${ }^{66}$ Thus, further research is needed to develop a model of the processes by which involvement and engagement in self-sufficiency promoting activities have their effects on parental well-being (also see discussion below).

Association of Parent Involvement in Head Start with Parent Involvement in Public School

Another aim of the present study was to explore the association of Head Start parent involvement with parents' involvement in public school. ${ }^{67}$ The following relationship emerged:

- Greater attendance at workshops and policy meetings during the Head Start year was associated with parents' helping more frequently and/or more frequently offering to help in the kindergarten classroom $(p<.05)$.

This finding is consistent with the notion that parents might feel more willing to offer help when they have more knowledge of appropriate practices for young children and child

${ }^{64}$ In the 1987 study, volunteering, attendance at workshops and policy meetings, and social services contacts all were correlated, and were included in one measure of involvement. In the present study, social service contacts are not correlated with the other types of involvement and are not included in the measure of overall involvement.

${ }^{65}$ Most high participators in the present study would have been classified as low participators in the earlier study.

${ }^{66}$ There was also post-hoc evidence that children's school-readiness may be adversely affected.

${ }^{67}$ Data on involvement in elementary school were collected for Agency One only. 
development milestones, the underlying content of many of the workshops in Head Start. Similarly, attending more policy committees and meetings might give them more confidence to approach teaching staff.

In summary, the relationships between involvement and positive outcomes for parents, the Head Start child, and the older sibling are complex, and many factors need to be considered. These results are suggestive that future research needs to concentrate on developing outcome-specific theoretical models of the effects of parent involvement.

\section{Relationships between Specific Types of Head Start Parent Involvement Activities and Outcomes}

The present study also explored the differential effects of specific types of parent involvement activities on outcomes. The two activity types explored in the present study (Volunteering; Workshops/Policy Meetings) contained different content, required different parental behaviors and interactions with others; and had different goals. Overall involvement appeared to account for more of the statistically significant relationships with outcomes than did the activity types. There are two possible explanations which may account for this: 1) There was more variability and range in overall involvement than in either Volunteering or Workshops/Policy Meetings, which resulted in more statistical power to detect statistically significant relationships; and 2) Because of the nature of Head Start record keeping, the activity types considered in the present study were still composites of somewhat different activities ${ }^{68}$ which might have different effects on outcomes.

Nevertheless, there were two findings which emerged at Agency One and were suggestive of differential effects of the two activity types:

- Volunteering in the classroom was associated with parents' helping the Head Start child learn school-readiness skills $(p<.05)$.

- Attendance at Workshops/Policy Meetings was associated with changes in parenting attitudes $(p<.10)$.

\footnotetext{
${ }^{68}$ For example, Volunteering included classroom, office, and kitchen activities as well as assistance on trips -activities which probably offer parents different skill benefits and experiences. interaction.
} 
The first finding may be understood in light of the fact that parents volunteer in their own child's classroom, providing the parent with information about her child's skills and capabilities, as well as with instructional techniques and knowledge of the types of skills that preschool children should be mastering. It is also possible that parents who are concerned about their children's school-readiness skills volunteer more in the classroom. The association of Workshops/Policy Meetings with parenting attitudes is interpreted in light of the parenting focus of many of the workshops.

Individual parent involvement activities differ in many ways. The next step in understanding the benefits of specific parent involvement activities requires the development of a theoretical model relating specific activities to specific outcomes, enhancing our ability to fully assess the benefits of Head Start. Testing such a model would require that Head Start staff and researchers work together to develop a record keeping procedure that would detail individual parent involvement activities. The testing of such a model would provide information to policy makers and educators about how to maximize the effectiveness of the parent component of the Head Start experience.

\section{Social Services Component of Head Start Parent Involvement}

The Social Services component of Head Start parent involvement differed from the other components, and was therefore, considered separately. Empirically, the present study found that Social Services utilization was not correlated with the other types of involvement, was predicted by different variables, and was not associated with positive outcomes. Thus, our data indicated that the social services component of Head Start was qualitatively different from the other components of parent involvement.

The predictors of Social Services utilization at Agency One ${ }^{69}$ accounted for approximately $10 \%$ of the variance. Greater utilization of social services during the Head Start year was associated with the following variables:

- Older parents $(\boldsymbol{p}<.05)$

- Less educated parents $(p<.01)$

- Parents who reported more self-sufficiency needs $(p<.05)$

${ }^{69}$ Social Services data were available on Agency One only. 
- Parents who helped their child learn more school-readiness skills $(p<.05)$

- Parents of Head Start children with more health problems $(p<.05)$

- Parents of older Head Start children $(p<.05)$

- Stricter parents $(p<.05)$

- Parents who spoke more frequently with their child about school $(p<.10)$

- Parents having an "older" older sibling $(p<.05)$

- Parents who reported the older sibling as having poorer school performance $(p<.10)$

Social Services did not have statistically significant positive associations with any of the parent, Head Start child or sibling outcomes.

The benefits of Social Services may be underestimated in the present study for several reasons: 1) Parents' level of utilization of social services was generally low, due to high caseloads and resultant staff overload; 2) Several appropriate outcomes (e.g., child health outcomes) were not assessed; 3) Parents who used Social Services more had more life problems which might not have been amenable to short-term change; and 4) The present study did not take into account the content of parents' social services contacts or the nature of the relationship between the family service worker and the parent.

The predictors data showed that the parents who were "worse off" to begin with appropriately utilized Social Services more often. However, this factor might have mitigated the relationship between Head Start involvement and outcomes. Although controlling for pretest scores controlled for one aspect of initial disadvantage, others which would influence the same outcome (e.g., negative life events occurring during the Head Start year) could not be statistically controlled.

As a first step in assessing the benefits of involvement on parent, child and older sibling outcomes, the present study examined the associations between number of hours of social services contacts and these outcomes. However, this study did not examine the content of parents' social services contacts, nor characteristics of the relationship between family service workers and the parent. The types of knowledge and aid given by staff during social service contacts varies greatly from parent to parent. Thus, in order to obtain a better notion of which outcomes are expected to improve for which subgroups of parents, future research should analyze the content of the social services visits. 
Another characteristic of the social services component of parent involvement not measured in the current study is the nature of the relationship between the Social Services worker and the parent developed over the course of the Head Start year. The quality and strength of this relationship may be important mediators of the benefits of Social Services contacts and should, therefore, be considered in future research on the impact of Head Start Social Services.

Although one major goal of the Social Services component is to aid in identifying opportunities for increasing self-sufficiency, the data did not reveal a statistically significant association with parents' self-sufficiency activities during the year following Head Start. ${ }^{70}$ Again, the families who utilized social services more may have had more life problems requiring a greater time period or more intervention for improvement to occur. Future research could examine the impact of social services on intermediate self-sufficiency promoting outcomes (e.g., following through on opportunities, keeping appointments for interviews, being on time) which might be more amenable to change. An evaluation of the full potential of the Social Services component will require an examination of the issues addressed in the social services contacts, and a reduction in staff caseloads so that more time can be allocated to individual families.

\section{The Next Steps: Fulfilling the Mission of Head Start as a Two-Generation Program}

As part of a two-generation program, the Head Start parent involvement component was designed to have multiple benefits for both the parent and Head Start child. To this end, parent involvement in Head Start has had several key goals: to promote parents' personal development and self-sufficiency; to promote their involvement in their children's development and school-readiness; and to help Head Start programs satisfy federal requirements for in-kind contributions which provide needed services to Head Start (Parker, Piotrkowski, Horn, \& Greene, 1995).

The present study provides tentative evidence of some of the ways in which the parent involvement component of Head Start may achieve these goals. Positive effects of

\footnotetext{
${ }^{70}$ Overall Involvement and Volunteering, however, did show relationships with aspects of self-sufficiency.
} 
involvement were found with respect to parental self-sufficiency, parents' involvement in their children's development and school-readiness, and aspects of Head Start child and sibling social competence. These findings provide additional evidence that Head Start is an institutional support system and effective two-generation program (Parker, Piotrkowski \& Peay, 1987).

The results also raise several issues regarding the conceptualization and measurement of parent involvement which suggest avenues by which future research can help shape an even more effective parent involvement program. These suggestions are especially timely in light of the current challenge of defining an effective Head Start parent involvement component for the 21 st century within the context of current economic and political realities, such as welfare reform (Parker, Piotrkowski, Horn \& Greene, 1995). Three related challenges for Head Start are highlighted by the results of the present study: how to customize services to meet the individual needs of each family; how to make it easier for parents to participate and benefit from involvement in Head Start; and, how to reconcile the new welfare-to-work demands on parents' time with Head Start's present requirements for parents' participation. These challenges raise two important issues regarding how to define and measure parent involvement so as to best study and understand its benefits for families.

The present study conceptualized and measured parent involvement as number of hours of participation. Although positive benefits of involvement were found using this metric, it is nevertheless only a first step because it does not take into account the quality of the parent's experience, which itself has many components. The skills parents may gain from different Head Start involvement activities, the perceived importance of the skill to the parent, the initiative required to engage in the activity, and parents' satisfaction and engagement with the activity, may influence the types and levels of positive outcomes for the Head Start family. ${ }^{71}$ There may be individual variation among parents in their responses to various involvement activities which affect how much they learn, what they

\footnotetext{
${ }^{71}$ We are currently conducting analyses which will shed light on the "black box" of involvement by examining the skill benefit derived from engagement in different parent involvement activities and the level of initiative required to engage in these activities.
} 
learn, and their satisfaction with their Head Start experience. The effectiveness of the parent's participation resulting in positive outcomes may also vary with the time during the year in which the parent participated. For example, if their experience is positive, parents who participate earlier in the year may have the opportunity and motivation to participate more later and thus benefit more from Head Start.

Another aspect of the quality of parents' participation may be the transactional or reciprocal nature of the parent's involvement with Parent Involvement/Social Services Staff, the Head Start child's teacher, other program administrators, and other Head Start parents and children. Individual differences in the fit between parents and teachers and between parents and staff may influence the parent's subjective experience of the program, her motivation to participate, and finally, the benefit derived from participation. Thus, conceptualizing involvement in terms of all of the processes involved in producing positive outcomes would be the next step in understanding how Head Start produces benefits for parents and children.

A significant definition and measurement issue relates to whether there is a minimum threshold of participation for involvement to have positive effects. In the present study, overall involvement at Agency One was substantially lower than in the previous study at the same Agency by Parker, Piotrkowski, and Peay (1987). Several factors not under the control of parents or the Head Start Agency may account for this. First, in the present harsher economic climate, a greater percentage of mothers at Agency One (33\% versus $18 \%$ in the earlier study at Agency One) were engaged in self-sufficiency promoting activities (working, attending school, job training) outside the Head Start center. In the present study, this engagement in self-sufficiency promoting activities was associated with a decrease in Head Start participation. Thus, these activities compete with traditional Head Start activities for parents' limited time and resources. Second, the insufficient funds for the parent workshops which are cornerstones of the Head Start parent involvement program (e.g., Exploring Parenting) meant that not all parents who wanted to attend were able to do so. Third, Social Services contacts were severely limited by unrealistically high staff caseloads. Because the earlier study (Parker, Piotrkowski, \& Peay, 1987) found that the high and medium involved, but not the low involved parents, benefited from involvement, it 
is very important for future research to determine whether there is a minimum threshold of involvement necessary for positive change. Although the present study found some benefits for the parent, Head Start child, and older sibling with current levels of involvement, these benefits might be increased if participation were greater.

Currently, there are no objective criteria available for categorizing parents as low, medium and high involved. These categories are usually defined relative to each study's own sample. Therefore, in the present study, the results of analyses which used the categorical measure of involvement ${ }^{72}$ may not generalize to other Head Start Agencies with different distributions and profiles of parent participation.

Another way of addressing the issue of how much involvement is needed for positive outcomes is to ask whether there is an optimum amount of involvement in light of other activities parents are engaged in, such that involvement above this level may result in overload, stress, or negative outcomes. This issue addresses the related challenges of how to reconcile participation in Head Start with the new welfare-to-work laws which place additional demands on parents' time, and how to make it easier for busy parents to participate in Head Start. Although a causal relationship cannot be established from our data, nevertheless, as stated earlier, the present study found an association between working, going to school, or being in job training and reduced participation in Head Start. Moreover, our post-hoc analyses were suggestive that the processes leading to positive outcomes and, therefore, the benefits of parent participation, may be different for Head Start parents who are also working, going to school, or in job training. For example, our post-hoc analyses revealed that parents who were engaged in self-sufficiency promoting activities were more depressed with greater involvement, whereas those who were not engaged were less depressed with greater involvement. For engaged parents, high levels of involvement, as currently defined may not always lead to positive outcomes. Policy makers need to take into account all of the activities in which parents are engaged when designing programs to benefit them.

\footnotetext{
${ }^{72}$ In the present study, these analyses included the post-hoc test of the non-linear relationships between involvement and outcomes; and the post-hoc test of the interaction of involvement and engagement in self-sufficiency promoting activities.
} 
One set of activities not currently taken into account in measuring parent involvement in Head Start is parent activities initiated by Head Start staff to be carried out at home (e.g., preparing food for a sale to raise money for Head Start activities; phoning other parents to schedule a meeting; engaging in a teacher-initiated learning activity with the Head Start child at home). This type of participation could not be assessed in the present study because records of parents' involvement in these types of activities are not kept by these (and most) Head Start programs. Researchers and Head Start staff need to develop a system for recording these activities so that their relationship to center activities and outcomes can be addressed. This type of participation may be favored by parents who are working, in job training or attending school during the day.

Another type of activity not usually assessed in evaluating the benefits of Head Start parent involvement is engagement in self-sufficiency promoting activities. The present study took the first steps in addressing the effects of this type of activity in combination with parent involvement. Understanding how engagement in self-sufficiency promoting activities combines with involvement to influence parent and Head Start child outcomes is important because many Head Start parents will be increasing their engagement in self-sufficiency promoting activities outside Head Start through mandatory participation in the new welfareto-work programs. The goals of these programs and of Head Start parent involvement are similar: Both aim to help parents engage in job training or education leading to paid employment. Parker, Piotrkowski, Horn and Greene (1995) suggest that helping parents to become self-sufficient is a component of the Head Start program that has not attained maximum effectiveness because of insufficient funds and staff training. Herr and Halpern (1991) suggest that creating a partnership between Head Start and welfare-to-work programs would provide both with additional resources that would help each fulfill its objective. For example, some welfare-to-work programs could be located at Head Start centers. Since Head Start is perceived by parents as a friendly, supportive environment which also provides good early-childhood child care, parents might be more comfortable attending welfare-to-work programs at their Head Start center. In addition, because parents' might spend less time and expense traveling between programs, they may have more time to learn the skills needed to attain self-sufficiency. Thus, this partnership might 
not only be cost-effective by reducing duplication of services, but could also increase the likelihood of parents' completing economic self-sufficiency programs. An additional benefit for Head Start could be the inclusion of these activities as in-kind services for the Head Start program, for better funding and accountability.

With demands on poor parents' time growing larger as a result of the welfare-towork provisions of current welfare reform, asking parents to substantially increase the number of hours of parent involvement in Head Start may be unrealistic. Thus, increasing the quality of the parent's Head Start experience may be crucial. This would accomplish two goals simultaneously: 1) making involvement in the Head Start program more satisfying and engaging to the parent, and 2) making involvement more effective in improving parents' lives.

In summary, helping parents receive maximum benefit from Head Start and attain self-sufficiency in part depends on understanding how all of the activities parents are involved in, both in and out of Head Start, together affect the parent and her family. Research can shed light on this process by providing a deeper understanding of the major forces impinging on the family. To summarize, research can inform policy by:

- Determining how current parent involvement activities produce positive outcomes in order to maximize their effectiveness.

- Identifying the benefits of different activities in order to customize programs to help the family attain self-sufficiency and a better quality of life.

- Developing additional involvement activities that are most effective in imparting the skills needed by parents and families to succeed in today's harsher economic climate.

- Identifying the support services needed by parents (e.g., child care) that will make it easier for them to take advantage of Head Start and other services and programs.

\section{Additional Methodological Considerations}

The present study's findings of a positive association of parent involvement with parent, Head Start child and sibling outcomes extends previous research because of the more rigorous methodology employed to test the hypotheses. The present study represents several key conceptual and methodological advances over previous parent involvement 
research because it: 1) measures parent involvement objectively from Head Start agency records rather than from retrospective parent reports; 2) examines a wide range of possible predictors of parent involvement; 3 ) evaluates the impact of parental participation on a wide range of outcomes and on family members other than the Head Start child; 4) explores the predictors of and impact of parent involvement by type of involvement activity as conceptualized in the Head Start Performance Standards; and 5) controls for initial differences on important demographic, contextual and personal characteristics of the Head Start parent, child and sibling, as well as for pretest differences on the outcomes. These methodological advances have provided a more rigorous evaluation of the effects of parent involvement than have previous studies. However, there were also some limitations in the design that qualify the present study's findings.

The two agencies in the study serve populations differing in ethnicity, acculturation, and neighborhood characteristics. Agency One parents were almost all Dominicans, largely born and educated outside the U.S.; whereas Agency Two parents were African-Americans, Hispanics, and Caucasians, born and educated in the U.S. Agency Two families reported statistically significantly more stress and negative life circumstances at the beginning of the Head Start year. The sample size at Agency One was almost twice that available at Agency Two. The results showed different patterns of participation and somewhat different outcomes of involvement in Agency One and Agency Two families, probably due to these initial differences as well as to differences in the Head Start parent involvement programs. Agency One emphasized volunteering in the classroom, whereas Agency Two emphasized workshops, trips and policy activities, and had a more comprehensive social services program. Because Social Services was an important component of the Agency Two intervention and because these data were not available to us, ${ }^{73}$ the benefits of the Agency Two parent involvement program was underestimated in the present study. The lesser number of statistically significant findings with respect to Agency Two, however, clearly does not reflect the many strengths of their high quality program. Agency Two parents and

\footnotetext{
${ }^{73}$ These data were not available to our research because much of the social services component was delivered at an outside mental health organization.
} 
children showed statistically significant improvement in several outcomes over the Head Start year. ${ }^{74}$

Because the two agencies differed in several ways, it was not possible to determine which factors may have contributed to the somewhat different patterns of results for the two agencies. The findings of the present study with respect to Agency One may not generalize to non-Dominicans born and educated in the U.S.

The results of the present study finding positive associations between parent involvement and parent, child, and sibling outcomes need to be interpreted in light of parents' self-selection into different levels of involvement. Because the present study employed a correlational design, there may be other unmeasured and uncontrolled variables which are actually causing the relationships between involvement and outcomes. Nevertheless, because this research does control for nine important demographic variables and pretest scores, it represents a major methodological advance over previous work, as well as a more rigorous test of the hypothesis.

Although pretest differences among parents were controlled in evaluating the benefits of Head Start, there was variability in extent and quality of parents' Head Start experiences prior to the beginning of the study which might make some parents more responsive to change during the study period. Number of years the parent was in Head Start was included in the set of demographic variables controlled for in evaluating the hypothesis of positive outcomes of Head Start, and it did not account for any of the statistically significant findings.

Head Start children also differed in whether they were in a full-time or a part-time program. At Agency One, preliminary analyses indicated that this variable did not account for differences in outcome scores. ${ }^{75}$ At Agency Two, because this variable was confounded with site and busing, its effects could not be isolated. ${ }^{76}$

\footnotetext{
${ }^{74}$ Parents overall reported less parenting aggravation, provision of more educationally relevant play materials, improved personal control and decreased depressive symptomatology at the end of the Head Start year.

${ }_{76}$ Approximately $11 \%$ of the Agency One children were in full-day classrooms because their parents were working.

${ }^{76}$ Approximately $45 \%$ of the children at Agency Two were in a full-day program.
} 


\section{Recommendations for Future Research}

The following recommendations for future research would add substantively to the ability to document and understand the ways in which parent involvement in Head Start has a positive impact on the lives of Head Start parents and their families. Such information is vital as Head Start moves into the 21st century.

\section{Analyses That Can be Conducted With Our Existing Data Set}

We plan to conduct the following analyses with our existing data set to further explore relationships between parent involvement and outcomes:

- Conduct cluster analyses to create combinations of parent involvement experiences (e.g., high in Workshops and policy but low in volunteering.) There may be types of participation combinations that have meaningfully different outcomes.

- Conduct post-hoc analyses using the different activity types, in addition to those already performed on overall. Control for important demographic variables in post hoc tests of the interaction between involvement and engagement in self-sufficiency promoting activities.

- Conduct regression analyses to examine possible interaction effects between involvement and other third variables (in addition to self-sufficiency). For example, involvement may have a greater positive association with aggravation for less strict . parents vs. stricter parents.

- Examine outcome analyses for specific subgroups of parent who may be more likely to benefit from Head Start parent involvement.

- Examine the effects of involvement on depression, after controlling for mastery and self-esteem. (s)

- Examine Dominican parents' outcome data (Agency One) from a cultural perspective, to better understand their culturally based beliefs, perceptions, and responses with regard to the major outcome constructs of the study (e.g., parentchild relationship, psychological well-being).

- Examine the content of parents' social services contacts and relate this content to improved outcomes. 
Suggestions for Future Research Studies That Could Build on This Study but Cannot be Done With the Existing Data Set:

Following is a summary of the directions in future research which we have recommended in this report to the Administration on Children, Youth, and Families:

- Refine the measure of involvement to take into account quality of experience for the parent.

- Identify additional predictors of involvement related to the characteristics of Head Start programs, other neighborhood variables and community obligations such as church, sibling's school, etc.

- Continue to develop a process model in which specific involvement activities are linked to specific types of outcomes.

- Examine the effects of involvement on additional outcomes that would best capture the benefits of the Head Start experience (e.g., child health outcomes).

- Examine the benefits of involvement for younger siblings of the Head Start child. Perhaps they would more likely benefit from changes within the family and the effects would be more pronounced.

- Examine the relationship between the family service worker, the Head Start teacher, other Head Start staff, and the parent as a mediator of improved outcomes.

\section{Policy and Programmatic Implications and Suggestions}

Following is a summary of the policy and programmatic recommendations made in this report:

- Redefine Head Start parent involvement to include activities initiated by the Head Start center to be carried out by the parent at home (e.g., reading to the Head Start child, volunteering on a Head Start fund-raising activity).

- Examine the feasibility of instituting state-level coordination between Head Start agencies and welfare-to-work programs so that Head Start agencies can offer on-site job training and educational activities leading to paid employment as part of their parent involvement component.

- Tailor programs to meet parents' specific goals, guided by a model of which aspects of parent involvement have which outcomes.

- Add services to decrease barriers to fuller participation in Head Start (e.g., child care, fullday program for Head Start child).

- Re-examine the needs and skills of the parents and how the program can address these by the types of participation activities offered. 


\section{REFERENCES}

ABT Associates. (October, 1988). Evaluation of Project Giant Step. (Technical Progress Report No. 4). Cambridge, MA: Author.

Achenbach, T.M. (1978). The child behavior profile: I: Boys aged 6-11. Journal of Consulting and Clinical Psychology, 46(3), 478-488.

Adkins, D.C. (1971). Programs of Head Start parent involvement in Hawaii: A section of the final report, 1969-1970. Hawaii: Education Research and Development Center, University of Honolulu.

Allen, W.R. (1978). The search for applicable theories of black family life. Journal of Marriage and the Family, 40, 117-129.

Alexander, K.L., \& Entwisle, D.R. (1988). Achievement in the first 2 years of school: Patterns and processes. Monographs of the Society for Research in Child Development, 53(6) (Serial No. 218).

Baker, A.J.L. (1993). Parent Involvement in School-Teacher Ratings Measure. New York: NCJW Center for the Child.

Boger, B.S. (November, 1969). Parents as primary change agents in experimental Head Start program of language intervention. Experimental Program Report. East Lansing, Michigan: Michigan State University Head Start Evaluation and Research Center. Michigan: Merrill Palmer Institute.

Bromley, K.E. (Ed.). (1972). Investigation of the effects of parent participation in Head Start. (Nos. ED 080 215-080 218). Denver: MIDCO Educational Associates.

Bronfenbrenner, U. (1986). Ecology of the family as a context for human development. Research Perspectives . Developmental Psychology, 22, 723-742.

Caldwell, B.M. (1974). Cooperative Preschool Inventory. Princeton: Educational Testing Service.

Chase, J.H., Weeldreyer, J.C., Cooper, C.S., \& Dunst, C.J. (September, 1986; Revised August, 1987). Parent Observation Scale for Assessing Parent-Professional Interaction. Morganton, N.C.: Project HAPPEN; Family, Infant and Preschool Program; Western Carolina Center.

Clark, R. (1993). Homework-focused parenting practices that positively affect student achievement. In N.F. Chavkin (Ed.), Families and schools in a pluralistic society. Albany, NY: SUNY University Press. 
Clarizio, H.F. (1968). Maternal attitude change associated with involvement in Project Head Start. Journal of Negro Education, 37, 106-113.

Cohen, J. (1969; Revised, 1977). Statistical Power Analysis for the Behavioral Sciences. New York: Academic Press.

Collins, R.C. (1993). Head Start: Steps toward a two-generation program strategy. Young Children, $\underline{48}(2), 25-33,72-73$.

Collins Management Consulting, Inc. (1990). Head Start research and evaluation: A blueprint for the future. Recommendations of the Advisory Board for the Head Start Evaluation Design Project. Contract No. 105-89-1610. U.S. Department of Health and Human Services. Washington, D.C.

Collins, R.C., \& Kinney, P.F. (1989). Head Start research and evaluation: Background and overview. Technical Paper prepared for Head Start evaluation design project. Vienna, VA: Collins Management Consulting, Inc.

Cook, T.D., \& Campbell, D.T. (1979). Quasi-experimentation. Chicago: Rand McNally.

Crnic, K.A., \& Greenberg, M.T. (1990). Minor parenting stresses with young children. Child Development, 61, 1628-1637.

Easterbrooks, M.A., \& Goldberg, W.A. (1984). Toddler development in the family: Impact of father involvement and parenting characteristics. Child Development, 55, 740-752.

Garfield. R.M. \& Abramson, D.M. (Eds.). (1994). Washington Heights/Inwood: The health of a community. New York: The Health of the Public Program at Columbia University.

Goldberg, W.A. \& Easterbrooks, M.A. (1984). Role of marital quality in toddler development. Developmental Psychology, 20, 504-514.

Gordon, I.J. (1969). Reaching the child through parent education: The Florida approach. Gainsville, FL: Institute for the Development of Human Resources.

Herr, T., \& Halpern, R. (1991). Changing what counts: Re-thinking the journey out of welfare. Evanston, IL: Center for Urban Affairs and Policy Research, Northwestern University.

Herr, T., Halpern, R., \& Majeske, R. (1995). Bridging the worlds of Head Start and welfare-to-work: Building a two-generation self-sufficiency program from the ground up. In S. Smith (Ed.). Two generation programs for families in poverty. Volume in the series, Advances in Applied Developmental Psychology. I. Sigel (Series Ed.). Norwood, NJ. Ablex. 
Hogan, A.E., Scott, K.G., \& Bauer, C.R. (1991). The adaptive social behavior inventory (ASBI): A new assessment of social competence in high risk three-year-olds. Unpublished manuscript. Miami: University of Miami.

Horn, W.F. (1993). Administration on Children, Youth and Families accomplishments: FY 1989 to FY 1993. Washington, DC: ACYF.

Hubbell, R. (1983). A review of Head Start research since 1970. Head Start Evaluation, Synthesis and Utilization Project. (No. OH 83-31184). Washington, DC: U.S. Government Printing Office.

Jacobs, S.H. (1970). Parent involvement in Project Head Start (Doctoral dissertation, University of Texas, 1970). Dissertation Abstract International, 31, (4-A), 1649.

Jones, E., \& Derman-Sparks, L. (1992). Meeting the challenge of diversity. Young Children, $\underline{47}(2), 12-18$.

Keith, T., Keith, P., Troutman, G., Bickley, P., Trivette, P., And Singh, K. (1993). Does parental involvement affect eighth-grade student achievement? Structural analysis of national data. School Psychology Review, 22, 474-496.

Lee, V., Brooks-Gunn, J., \& Schnur, E. (1988). Does Head Start work? A one year follow up comparison of disadvantaged children attending Head Start, no preschool, and other preschool programs. Developmental Psychology, 24, 210-222.

McKey, R.H., Condelli, L., Ganson, H., Barrett, B.J., McConkey, C., \& Plantz, M.C. (1985). The impact of Head Start on children, families and communities. Final Report of the Head Start Evaluation, Synthesis and Utilization project. (No. 105-81-C-026). Washington, DC: CRS, Inc.

Melon, R. (Ed.). (1995). A collection of original poems, stories and letters by the parents of children attending Washington County Head Start. New York, NY: Washington County Child Care Programs.

Meisels, S.J., \& Wiske, M. S. (1988). Early Screening Inventory Parent Questionnaire. New York: Teachers College Press.

Miller, S., Manhal, M., and Mee, L. (1991). Parental beliefs, parental accuracy, and children's cognitive performance: A search for causal relations. Developmental Psychology, 27(2), 267-276.

National Council of Jewish Women Center for the Child. (1991). About Your Older Child Questionnaire. Unpublished measure. New York: Author. 
NCJW Center for the Child. (1992). Intake/Family Profile. Unpublished measure. New York: Author.

NCJW Center for the Child. (1992). Parent Involvement Information Protocol. Unpublished measure. New York: Author.

National Head Start Association. (May, 1990). Head Start: The Nation's Pride , A Nation's Challenge. Recommendations for Head Start in the 1990's. The Report of the Silver Ribbon Pannel. Alexandria, VA: Author.

Norbeck, J.S. (1984). Modification of life events questionnaires for use with female respondents. Research in Nursing and Health, $\underline{7}$, 61-71.

O'Brien, P. (1985). Global self-esteem scales: Unidemensional or multidimensional? Psychological Report, 57, 383-389.

Oyemade, U., Washington, V., \& Gullo D. (1989). The relationship between Head Start parent involvement and economic self-sufficiency of Head Start families. Journal of

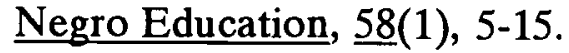

Parker, F.L., Piotrkowski, C.S., Horn, W.F., \& Greene, S. (1995). The challenge for Head Start: Realizing its vision as a two-generation program. In S. Smith (Ed.). Two generation programs for families in poverty. Volume in the series, Advances in Applied Developmental Psychology. I. Sigel (Series Ed.). Norwood, NJ. Ablex.

Parker, F.L., Kessler-Sklar, Piotrkowski, C.S., S., Peay, L., Clark, B., \& Baker, A.J.L. (1993). Telephone Interview Protocol. Unpublished measure.

Parker, F.L., Piotrkowski, C.S., Yoshikawa, H., Clark, B. \& Peay, L. (1991). Barriers to Head Start Parent Participation Survey. Unpublished measure.

Parker, F.L., Piotrkowski, C.S., \& Peay, L. (1987). Head Start as a social support for mothers: The psychological benefits of involvement. American Journal of Orthopsychiatry, 57(2), 220-233.

Pascoe,J.M., \& French, J. (1990). The reliability and validity of the Maternal Social Support Index for primiparous mothers. Farm Medicine, 22(3), 228-230.

Pascoe, J.M., Ialongo, N., Horn, W.F., \& Reinhart, M.A. (1988). The reliability and validity of the maternal social support index. Family Medicine, 20, 271-276.

Pearlin, L., \& Schooler, C. (1978). The structure of coping. Journal of Health and Social Behavior, 19, 2-21. 
Phillips, L. (1992). Parent involvement: Relationships of expectations, goals, and activities to student achievement among minority, socioeconomic, and gender groups. Paper presented at the annual meeting of the American Educational Research Association.

Piotrkowski, C.S., \& Kessler-Sklar, S.L. (1996). Welfare reform and access to family-supportive benefits in the workplace. American Journal of Orthopsychiatry, 538547.

Powell, D.R. (1984). Social support and demographic predictors of length of participation in a parent education program. Journal of Community Psychology, 11, 1-24.

Radloff, L. (1977). The CES-D scale: A self-report depression scale for research in the general population. Applied Psychological Measurement, 1, 385-401.

Rauh, V.A., Wasserman, G.A., \& Brunelli, S.A. (1990). Determinants of maternal child rearing attitudes. Journal of the American Academy of Child and Adolescent Psychiatry, $\underline{29}(3), 375-381$.

Rauh, V., Velez, C.N., \& Lederman, S.A. (1990). A comparison of lifestyles during pregnancy between two groups of Puerto Rican women: Island versus New York City residents. Presented at Brown University conference. Health and Development of Puerto Rican Mothers and Children in the Mainland.

Replogle, E.M. (1995). Head Start as a family support: Renewing a community ethic. Cambridge, MA: Harvard Family Research Project.

Reynolds, A. \& Gill, S. (1994). The role of parental perspectives in the school adjustment of inner-city Black children. Journal of Youth and Adolescence, 23, 671-694.

Rosenberg, M. (1965). Society and the adolescent self-image. Princeton, NJ: Princeton University Press.

Schiamberg, L. and Chin, C. (1986). The influence of family on educational and occupational achievement. Paper presented at the annual meeting of the American Association for the Advancement of Science, Philadelphia, PA.

Slaughter, D.T., Lindsey, R.W., Nakagawa, K., \& Kuehne, V.S. (1989). Who gets involved? Head Start mothers as persons. The Journal of Negro Education, 58(1), 16-29.

Stack, C. (1974). All our kin: Strategies for survival in a Black community. New York: Harper \& Row.

Staples, R. (1971). Toward a sociology of the Black family: A theoretical and methodological assessment. Journal of Marriage and the Family, 33, 119-138. 
Swift, M. (1968). Language style of the lower class mother: A preliminary study of a therapeutic technique. Philadelphia, PA: Hahnemann Community Mental Health Center.

U.S. Department of Health and Human Services, Administration on Children, Youth and Families. (1996). A annotated bibliography of Head Start research: 1985-1996. Washington, DC. U.S. Government Printing Office.

U.S. Department of Health and Human Services, Administration on Children, Youth and Families. (1995, February). Project Head Start Statistical Fact Sheet. Washington, DC: U.S. Government Printing Office.

U.S. Department of Health and Human Services. (1987). Looking at life. (USGPO 1987175-782). Washington, DC: U.S. Government Printing Office.

U.S. Department of Health and Human Services. (1977). Head Start Assessment. Washington DC: U.S. Government Printing Office.

U.S. Department of Health and Human Services. (1976). Exploring parenting. (DHHS Publication No. 82-31134). Washington DC: U.S. Government Printing Office.

U.S. Department of Health and Human Services. (1975; Reissued, 1984; Reprinted 1992; Revised, 1996). Head Start Program Performance Standards. (DHHS Publication No. OHDS 84-31131). Washington, DC: U.S. Government Printing Office.

Valentine, J., \& Stark, E. (1979). The social context of parent involvement in Head Start. In E. Zigler and J. Valentine (Eds.), Project Head Start: A legacy of the war on poverty (pp. 291-313). New York: Free Press.

Wang, J. \& Wildman, L. (1994). The effects of family commitment in education on student achievement in seventh grade mathematics. Education, 115, 317-319.

Zigler, E. (1978). The effect of Head Start: Another look. Educational Psychology, $\underline{13}$, 71-77.

Zigler, E., \& Berman, W. (1983). Discerning the future of early childhood intervention. American Psychologist, $\underline{38}, 894-906$.

Zigler, E., \& Muenchow, S. (1992). Head Start: The inside story of America's most successful educational experiment. New York: Basic Books.

Zigler, E., \& Trickett, P. (1978). IQ, social competence, and evaluation of early childhood education programs. American Psychologist, $\underline{33}$, 789-798.

Zigler, E., \& Valentine, J. (Eds.). (1979). Project Head Start: A legacy of the war on poverty. New York: Free Press. 


\section{LIST OF ITEMS:}

1) Table 1: Potential Predictors of Parent Involvement

2) Table 2: Demographic \& Contextual Characteristics of Head Start Samples at Pretest

3) Table 3: Pretest Variables Tested in Attrition Analyses

4) Table 4: Description of Parents' Participation

5) Table 5: Intercorrelations Among the Parent Involvement Variables

6) Table 6: Predictors of Parents' Involvement by Type of Involvement

7) Table 7: Analyses Comparing Pretest and Posttest Scores on Parent Outcomes

8) Table 8: Partial Correlations Between Parent Involvement and Parent Outcomes Controlling for Pretest

9) Table 9: T-tests Comparing Head Start Child Pretests to Post Head Start Outcomes

10) Table 10: Partial Correlations Between Parent Involvement and child Outcomes Controlling for Pretest

11) Table 11: T-tests Comparing Older Siblings' Pretest and One Year Follow-up CBCL Scores

12) Table 12: Partial Correlations Between Parent Involvement and CBCL Sibling Outcomes

13) Table 13: Description of Parents' Participation

14) Table 14: Intercorrelations Among The Parent Involvement Variables

15) Table 15: T-tests Comparing Parent Pretests To Post Head Start Parent Outcomes

16) Table 16: Partial Correlations Between Parent Involvement and Parent Outcomes Controlling for Pretest

17) Table 17: T-tests Comparing Pretests to Post Head Start Child Outcomes

18) Table 18: Partial Correlations Between Parent Involvement and Child Outcomes Controlling for Pretest

19) Figure 1: Depression

20) Figure 2: Cooperative Preschool Inventory Scores

21) Figure 3: Parental Encouragement of The Head Start Child's Independence 


\section{MOTHER'S CHARACTERISTICS:}

1) Number of years in U.S. (NOYRSUS)

2) Acculturation factors (born, educated in U.S., speaks English) (ACCULT1)

3) Highest grade mother completed (HIGRADEB)

4) Age (MOTHRAGE)

5) Pregnancy (PRGORBAB)

6) Number of life changing events or experiences in prior year (TOPSTRES)

7) Self esteem (LGSEPRI)

8) Personal control (MASTPR1)

9) Depression (SMCESPR1)

10) Number of years parent was in Head Start (YRSPARHS)

11) Need for greater economic self-sufficiency (NEEDY1)

12) Engagement in self-sufficiency promoting activities during the Head Start year (working, attending school or job training) (BUSYMAN)

\section{PARENTING CHARACTERISTICS:}

A. Parenting Daily Hassles

13) Frequency of hassles (PDHFRPR1)

14) Intensity of hassles (PDHINPR1)

B. Parenting Attitudes Toward Childrearing

15) Parental attitudes towards the expression of affection (Warmth) (PACWAPR1)

16) Parental attitudes towards children's autonomous behavior (Encouragement of Independence) (PACENPR1)

17) Parental attitudes towards discipline and self-control (Strictness) (PACSTPR1)

18) Parental feelings of being annoyed or upset by their children's behavior (Aggravation) (PACAGPR1)

C. Home Learning Environment

19) How frequently child helps with household tasks (HTASKPR1)

20) Number of play materials in the home (PLAMAPR1)

21) Number of school-readiness skills parent has helped child learn (CHLRNPR1)

22) How frequently parent and child talk about school (DOTGRPR1)

23) Parent's expectations for child's school performance (EXCHPR1A)

\section{HEAD START CHILD CHARACTERISTICS:}

24) Age (CHILDAGE)

25) Gender (CHILDSEX)

26) Birth difficulties (EPQMDPR1)

27) Not achieving developmental milestones (EPQDVPRI)

28) Health-related problems since birth (EPQHLPR1)

29) Number of years child has been in Head Start (HSCNOYRS) 


\section{Table 1 (Continued)}

IV. OLDER SIBLING CHARACTERISTICS:

30) Age (SIBAGE)

31) Gender (SIBSEX)

32) Birth problems (SQMDPR1)

33) Health-related problems (SQHLPR1)

34) $\mathrm{CBCL}$ activity competence (CBCAC)

35) CBCL school competence ( $C B C S L$ )

36) CBCL social competence ( $C B C S O$ )

37) $\mathrm{CBCL}$ total competence (CBCTC)

\section{FAMILY CHARACTERISTICS:}

38) Number of other children in the home (TOTCHA)

39) Mother's social support (MSSSPR1)

40-42) Ethnicity (ETHNICA, ETHNICB, ETHNICC)

\section{COMMUNITY CHARACTERISTICS:}

43) Perceived neighborhood safety (NSAFETYA)

Because $99 \%$ of the families in Agency One were Dominican, ethnicity was considered as a predictor for Agency Two only. Ethnicity was coded as a set of three dummy variables: (a) African-American vs not; (b) Hispanic
vs not; and (c) Caucasian vs not. 


\section{Table 2 \\ DEMOGRAPHIC \& CONTEXTUAL CHARACTERISTICS \\ OF HEAD START SAMPLES AT PRETEST \\ (COHORT I: AGENCIES ONE AND TWO)}

VARIABLES

I. MOTHER

A. Ethnicity:

1. African-American

2. Euro-American

3. Hispanic

4. Other

B. Mother's Country of Birth:

USA

Other

C. Number of Years in United States:

Average

14

D. Acculturation Factors:

(born, educated US, speaks English)

0
1
2
3

25

7

20

E. Education:

Grade 1-8

Grade 9-11

Grade 12 or GED

College or other Post High

School Education

F. Age:

Average

G. Pregnancy or Birth in Head Start Year:

60

18

18

4

8

82

18

27

1

0

11

80

3

33

50

15

31

20
AGENCY TWO (a) $(\mathrm{N}=103)$

\section{PERCENT or AVERAGE}

II. HEAD START CHILDREN AND OLDER SIBLINGS

A. Head Start Child:

1. Years in Head Start 1

2

2. Gender

Girls

Boys

3. Age

Average

B. Older Sibling: ${ }^{(b)}$

1. Gender

Girls

Boys

2. Age 
I. MOTHER'S CHARACTERISTICS:

1) Highest grade completed

2) Age

3) Number of life changing events or experiences in prior year

4) Personal control

5) Depression

6) Self esteem

II. PARENTING CHARACTERISTICS:

A. Parenting Daily Hassles

1) Number of hassles

2) Intensity of hassles

B. Parenting Attitudes Toward Childrearing

1) Parental attitudes towards the expression of affection (Warmth)

2) Parental attitudes towards children's autonomous behavior (Encouragement of Independence)

3) Parental attitudes towards discipline and self-control (Strictness)

4) Parental feelings of being annoyed or upset by their children's behavior (Aggravation)

C. Home Learning Environment

1) How frequently child helps with household tasks

2) Number of play materials in the home

3) Number of school-readiness skills parent has helped child learn

4) How frequently parent and child talk about school

5) Parent's expectations for child's school performance

\section{HEAD START CHILD CHARACTERISTICS:}

1) Age

2) Gender

3) Birth difficulties

4) Not achieving developmental milestones

5) Health-related problems

IV. OLDER SIBLING CHARACTERISTICS:

1) Age

2) Gender

3) Birth problems

4) Health-related problems

5) $\mathrm{CBCL}$ activity competence

6) CBCL school competence

7) CBCL social competence

8) CBCL total competence

V. FAMILY CHARACTERISTICS:

1) Number of other children in the home

2) Mother's social support 


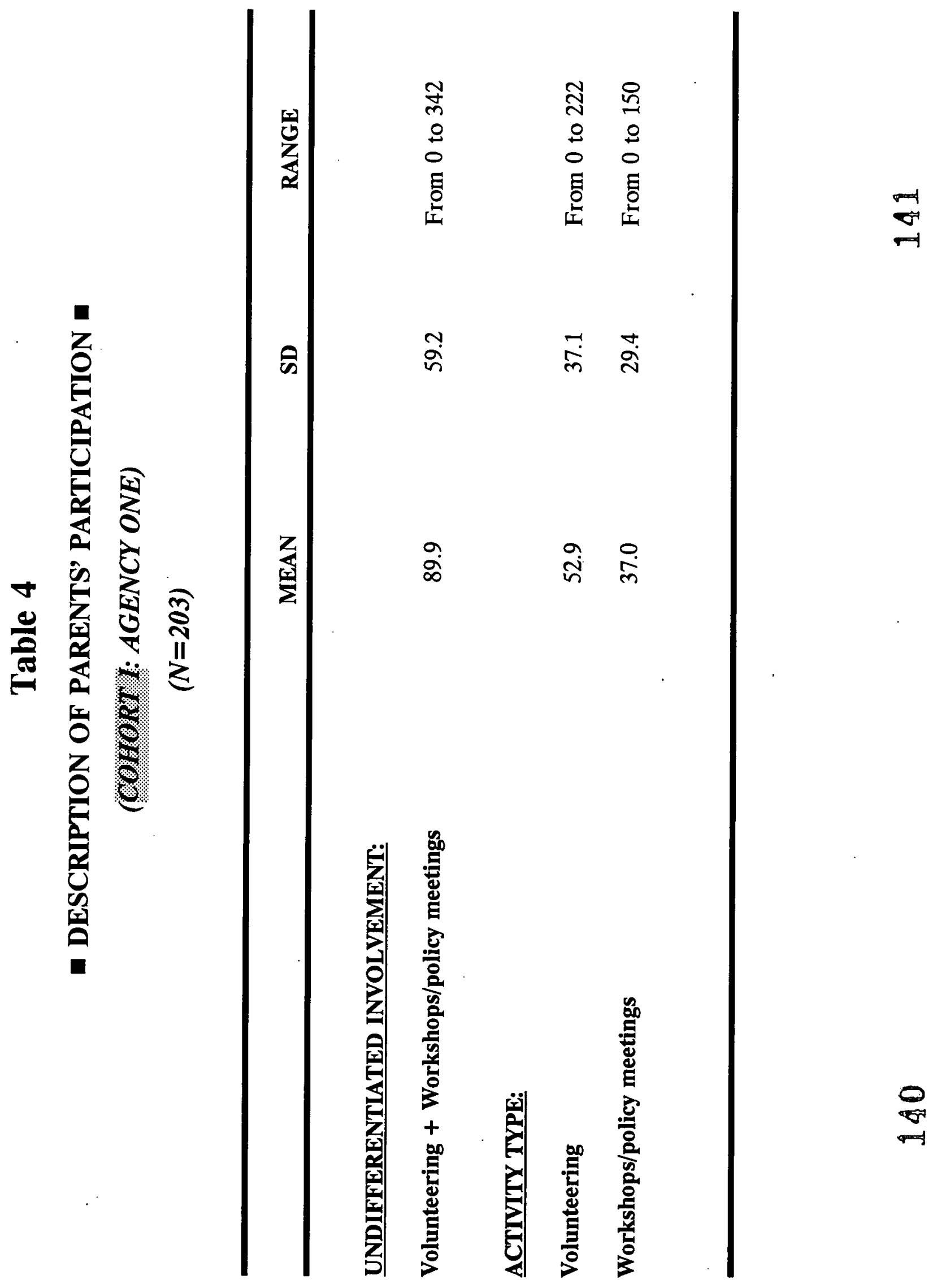




\section{Table 5}

INTERCORRELATIONS AMONG

THE PARENT INVOLVEMENT VARIABLES

(COHORT : AGENCY ONE)

$(N=203)$

WORKSHOPS/

VOLUNTEERING POLICY MEETINGS

\section{UNDIFFERENTIATED INVOLVEMENT:}

1. Volunteering + wokshops/policy meetings

$$
.91 * * *
$$

$.86 * * *$

\section{ACTIVITY TYPE:}

2. Volunteering

$-.-58 * * *$

3. Workshops/policy meetings

$* * *=p<.001$ 


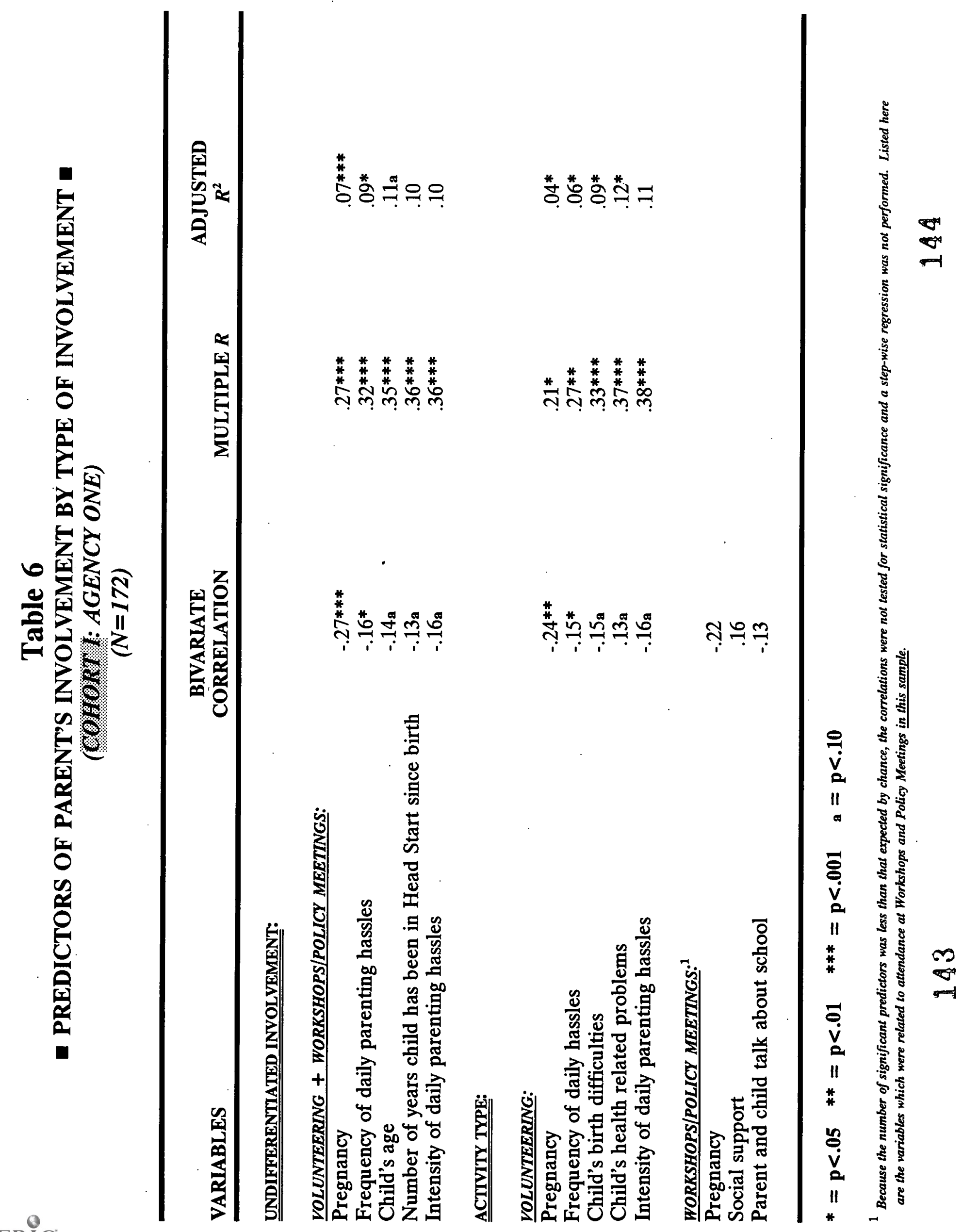




\section{Table 7}

ANALYSES COMPARING PRETEST AND

POSTTEST SCORES ON PARENT OUTCOMES

(COHORT I: AGENCY ONE)

$(N=172)$

OUTCOME

PARENT-CHILD RELATIONSHIP:

1) Encouragement of independence $\underline{\mathrm{M}}(\underline{\mathrm{SD}})$

2) Strictness

$\underline{\mathrm{M}}(\underline{\mathrm{SD}})$

3) Aggravation

$\underline{\mathrm{M}}(\underline{\mathrm{SD}})$

4) Frequency of parenting daily hassles $\underline{\mathrm{M}}(\underline{\mathrm{SD}})$

HOME LEARNING ENVIRONMENT:

1) Number of play materials at home $\underline{\mathrm{M}}(\underline{\mathrm{SD}})$

2) Number of school-readiness skills parent has helped child learn $\underline{\mathrm{M}}(\underline{\mathrm{SD}})$

3) Parent's expectations for child's school performance $\underline{\mathrm{M}}(\underline{\mathrm{SD}})$

PARENTAL WELL-BEING:

1) Personal Control

$\underline{\mathrm{M}}(\underline{\mathrm{SD}})$

2) Depression

$\underline{\mathrm{M}}(\underline{\mathrm{SD}})$
169
$4.7(0.8)$
$4.9(0.7)$
$-3.15^{* *}$

169

$3.2(0.7)$

$3.3(0.7)$

169

$3.4(0.6)$

$3.4(0.6)$

.57

161

$9.3(5.1)$

$9.6(5.9)$

$-.96$

170

$6.6(2.3)$

$8.0(2.3)$

$-8.61 * * *$

170

$8.1(2.3)$

$9.1(2.0)$

$-6.22 * * *$

162

$1.4(0.6) \quad 1.3(0.6)$

.67

167

$2.8(0.4)$

$3.0(0.4)$

$-3.92 * * *$

170

$15.7(10.6) \quad 13.4(9.8)$

$2.88 * *$
PROPORTION OF SAMPLE

HEAD START YEAR FOLLOW-UP CHI SQUARE

PARENT SELF-SUFFICIENCY ACTIVITIES:

1) No paid job in year after Head Start

2) Not pregnant in year after Head Start

$82 \%$

$87 \%$

3) No education or job training in year after Head Start

$77 \%$

$73 \%$

$90 \%$

$24 \%$

$10.2 * *$

0.1

$33.1 * *$

$* *=p<.01 ; \quad * * *=p<.001$ 

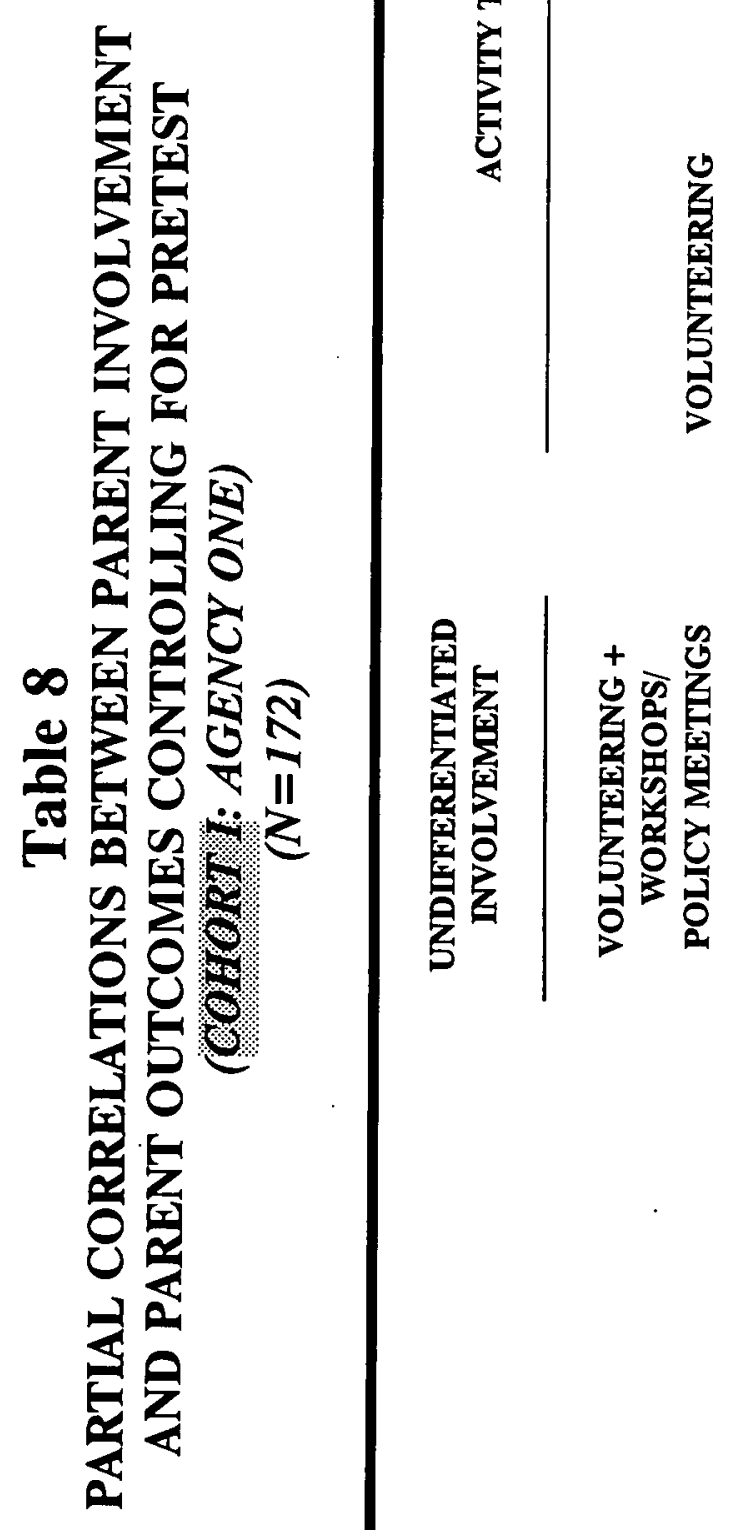

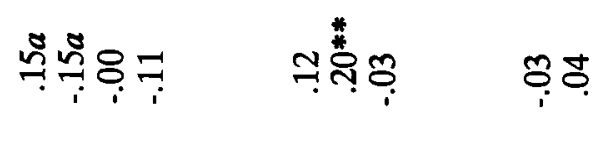

๙ุํํ용

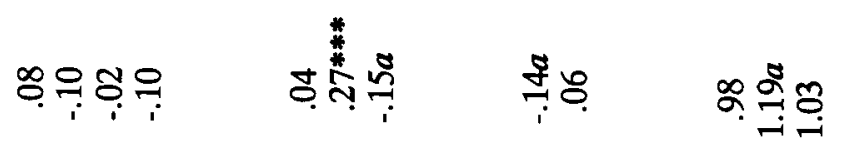

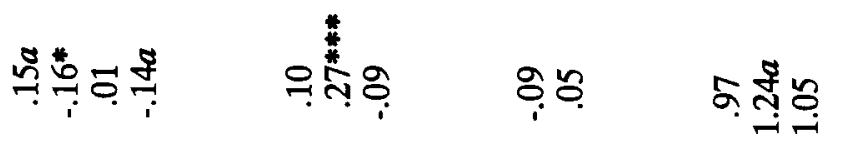

莺

융

욤

斗

氙

i்

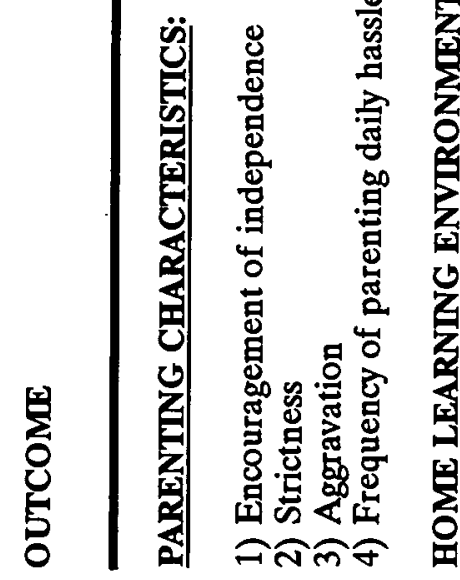

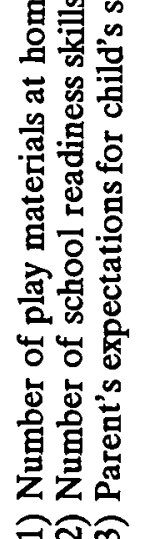

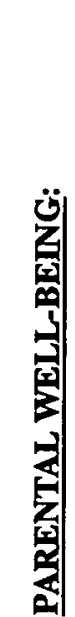
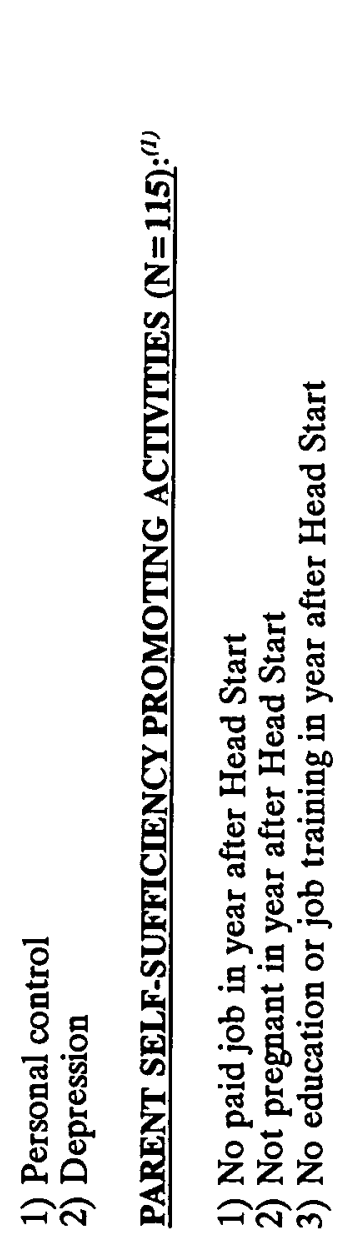


\section{Table 9}

\section{T-TESTS COMPARING HEAD START CHILD PRETESTS TO POST HEAD START OUTCOMES}

(COHORT I: AGENCY ONE)

\section{OUTCOME}

1) Parent's Report of Child's Disruptive or Demanding Behavior

$$
\underline{\mathrm{M}}
$$

2) Parent's Report of Child's Cooperativeness/

Compliance With Rules, Directives

$$
\underline{\mathrm{MD}}
$$

3) Cooperative Preschool Inventory Total

$$
\underline{\mathrm{MD}}
$$

df

PRETEST
143

$\begin{array}{lcl}12.6 & 11.7 & 3.57^{* * *} \\ 2.8 & 2.5 & \end{array}$

160

$\begin{array}{ccc}24.5 & 25.1 & -2.15^{*} \\ 3.2 & 3.2 & \end{array}$

128

$\begin{array}{cc}27.4 & 36.7 \\ 9.7 & 9.1\end{array}$

$$
*=p<.05 \quad * * *=p<.001
$$




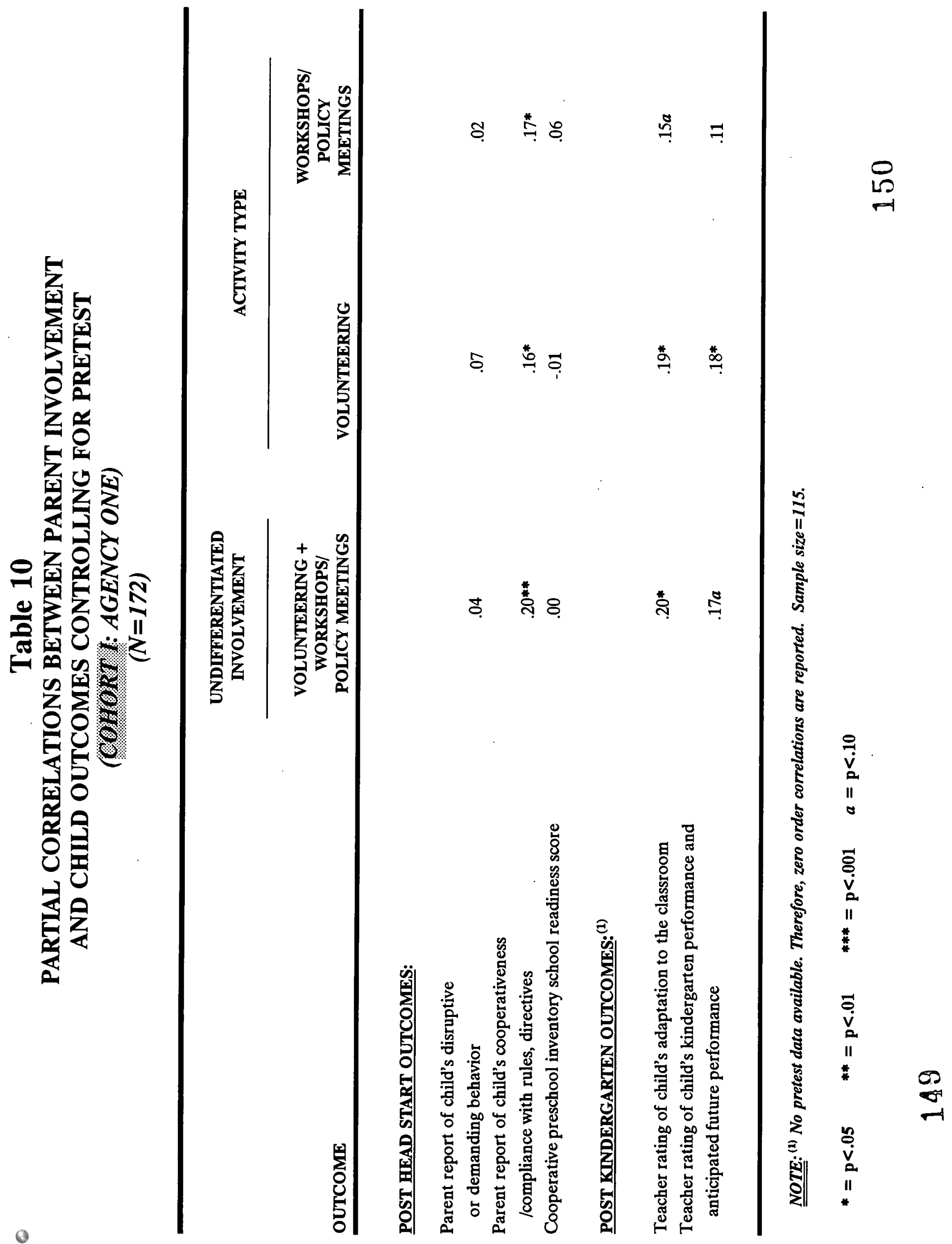




\section{Table 11}

\section{T-TESTS COMPARING OLDER SIBLINGS'}

PRETEST AND ONE YEAR FOLLOW-UP CBCL SCORES

(COHORT I: AGENCY ONE)

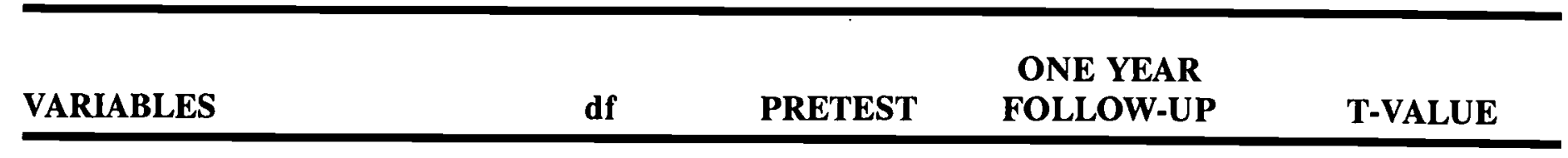

ACTIVITIES SCALE:

Mean

SD

SCHOOL SCALE:

Mean

SD

36

5.06

.91

4.71

1.11

3.88

1.41

1.99
.67

SOCIAL SCALE:

OVERALl SOCIAL COMPETENCE:
1.11
Mean
43
4.43
4.33
1.62
1.45
SD

.35

Mean

35

13.98

2.97

13.01

2.93

$1.81 a$

SD

$a=\mathrm{p}<.10 ; \quad *=\mathrm{p}<.05 ; \quad * *=\mathrm{p}<.01 ; \quad * * *=\mathrm{p}<.001$ 


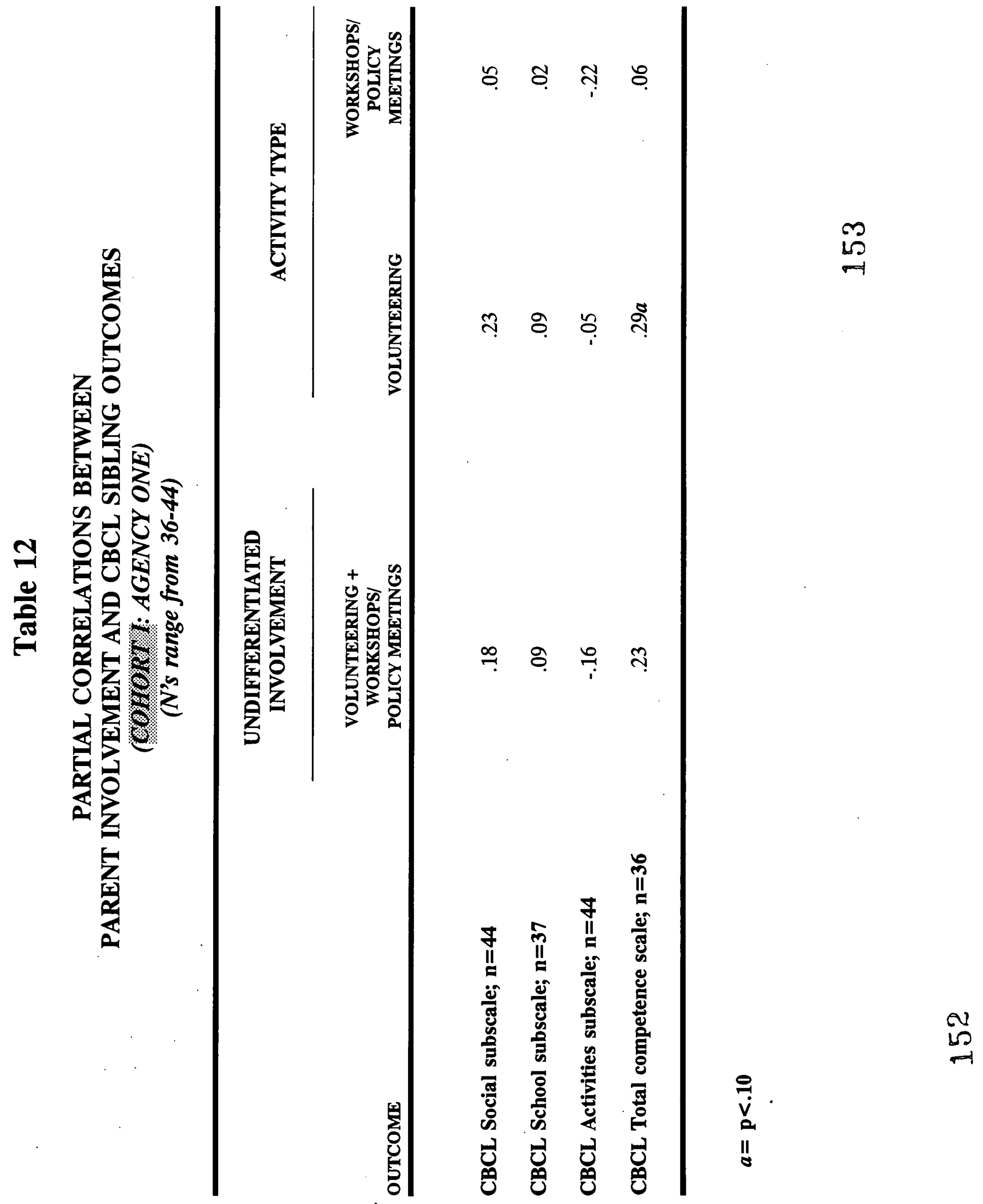




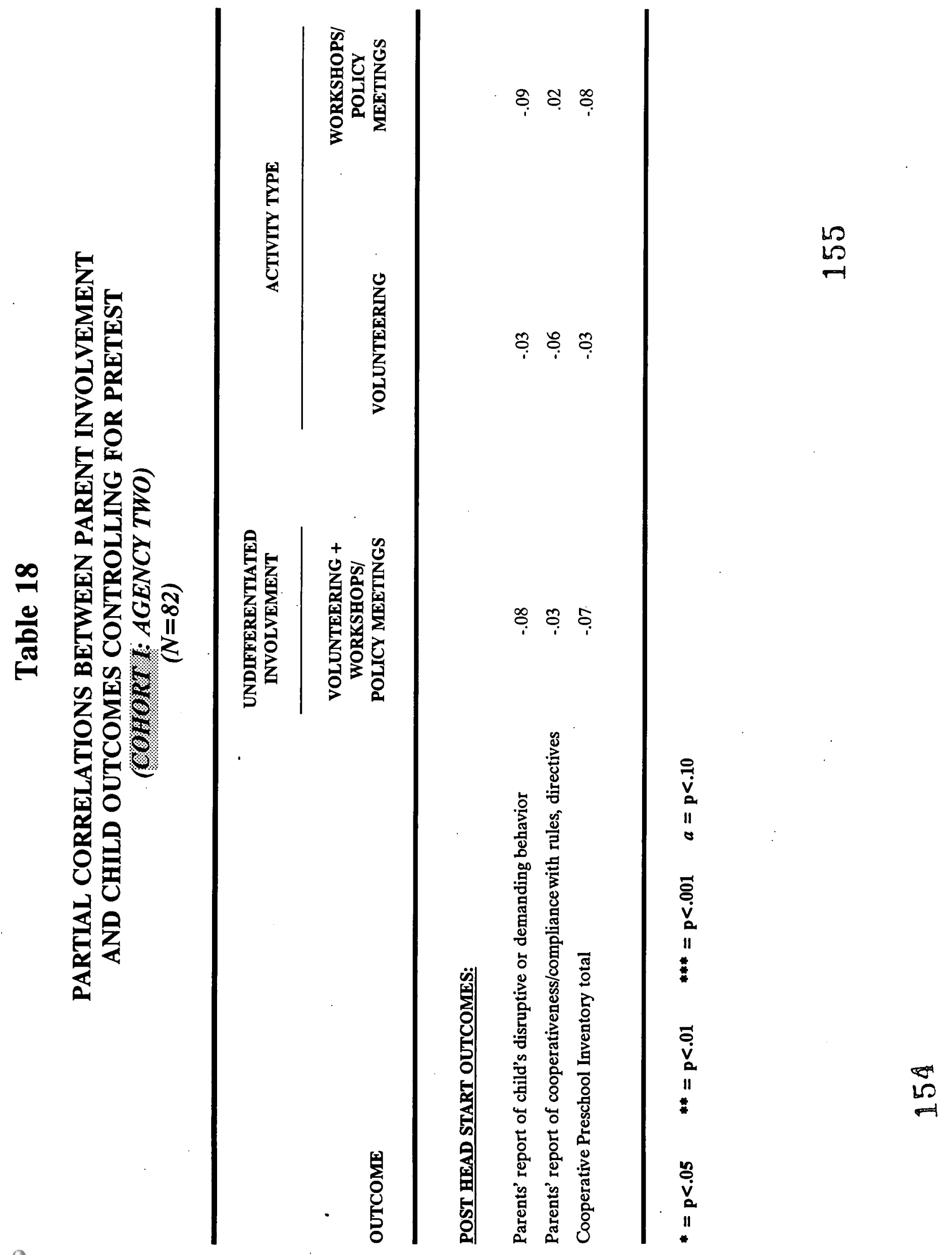




\section{Table 13}

ם DESCRIPTION OF PARENTS' PARTICIPATION

(OOHORT I: AGENCY TWO)

$$
(N=103)
$$

\begin{tabular}{llll}
\hline & MEAN & SD & RANGE \\
\hline $\begin{array}{l}\text { UNDIFFERENTIATED INVOLVEMENT: } \\
\text { Volunteering + workshops } \\
\text { /policy meetings }\end{array}$ & 53.8 & 82.8 & From 0 to 419 \\
$\begin{array}{l}\text { ACTIVITY TYPE: } \\
\text { Volunteering }\end{array}$ & & & \\
Workshops/policy meetings & 26.4 & 42.6 & From 0 to 299 \\
\end{tabular}




\section{Table 14}

\section{INTERCORRELATIONS AMONG THE PARENT INVOLVEMENT VARIABLES \\ (COHORT I: AGENCY TWO) \\ $(N=103)$}

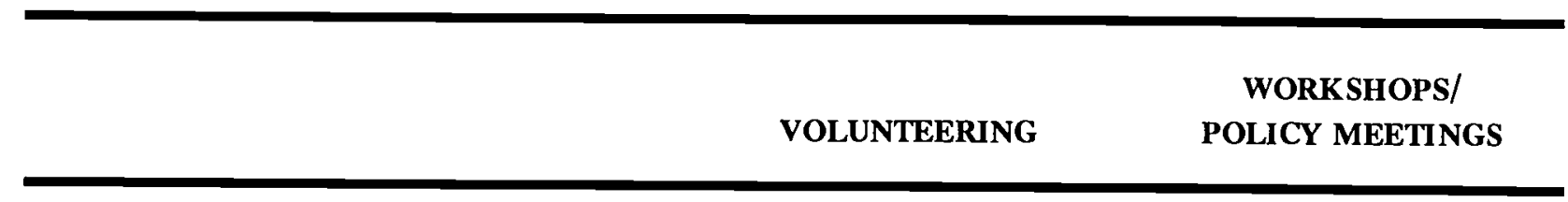

UNDIFFERENTIATED INVOLVEMENT:

Volunteering + workshops /policy meetings

$.89 * * *$

$---$
$.63^{* * *}$

Workshops/policy meetings

$* * *=p<: 001$ 


\section{Table 15}

\section{T-TESTS COMPARING PARENT PRETESTS \\ TO POST HEAD START PARENT OUTCOMES \\ (COWORT I: AGENCY TWO) \\ $(N=82)$}

PRETEST

POSTTEST

T-VALUE

\section{PARENT-CHILD RELATIONSHIP:}

1) Encouragement of independence $\underline{\mathrm{M}}(\underline{\mathrm{SD}})$

78

$4.7(0.7)$

$4.8(0.7)$

$-.32$

2) Strictness

$\underline{\mathrm{M}}(\underline{\mathrm{SD}})$

78

$3.2(0.7)$

$3.2(0.6)$

$-1.16$

3) Aggravation

78

$\underline{\mathrm{M}}(\underline{\mathrm{SD}})$

$3.3(0.7)$

$3.2(0.7)$

$1.95 a$

4) Frequency of parenting daily hassles $\underline{M}(\underline{S D})$

76

$12.0(6.1)$

$12.2(6.6)$

$-.24$

HOME LEARNING ENVIRONMENT:

1) Number of play materials at home $\underline{M}(\underline{S D})$

73

$0.6(0.2)$

$0.7(0.2)$

$-4.38 * * *$

2) Number of school-readiness skills parent has helped child learn $\underline{\mathrm{M}}(\underline{\mathrm{SD}})$

$0.9(0.2)$

$0.9(0.2)$

3) Parent's expectations for child's school performance $\underline{\mathrm{M}}(\underline{\mathrm{SD}})$

$1.4(0.6)$

$1.5(0.6)$

\section{PARENTAL WELL-BEING}

1) Personal Control

$\underline{M}(\underline{S D})$

2) Depression

$\underline{\mathrm{M}}(\underline{\mathrm{SD}})$
80

$3.2(0.5)$

$-2.80 * *$

$$
a=\mathrm{p}<.10 \quad * *=\mathrm{p}<.01 \quad * * *=\mathrm{p}<.001
$$
$15.2(10.9)$
$12.6(9.9)$
$2.85^{* *}$ 


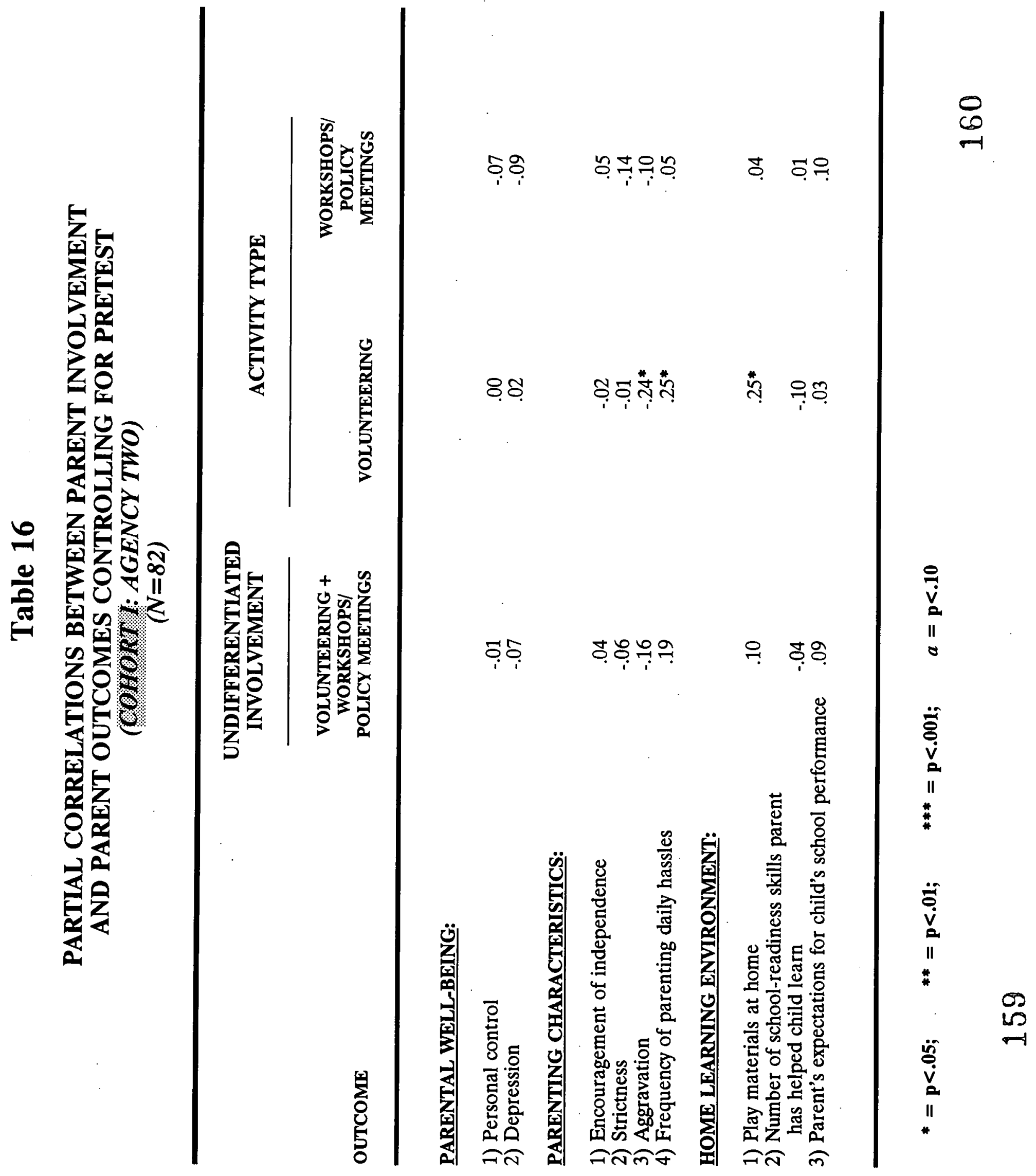




\section{Table 17}

\section{T-TESTS COMPARING PRETESTS \\ TO POST HEAD START CHILD OUTCOMES \\ (COHORT I: AGENCY TWO) \\ $(N=82)$}

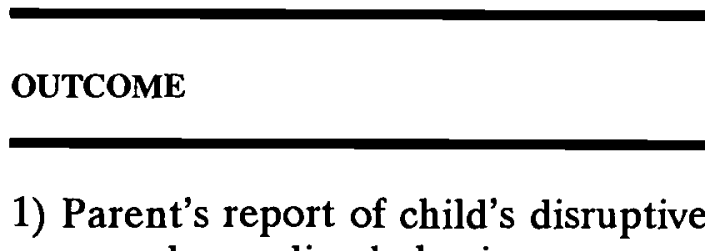
or demanding behavior

M

$\underline{\mathrm{SD}}$

2) Parent's report of child's cooperativeness/ compliance with rules, directives

$\underline{\mathrm{M}}$

$\underline{\mathrm{SD}}$

3) Cooperative preschool inventory total

M

$\underline{\mathrm{SD}}$
63

$\begin{array}{rr}10.8 & 11.2 \\ 2.3 & 2.5\end{array}$

1.65

72

24.4

24.4

.08

3.3

3.2

75

34.8

11.5

42.8

10.2

$* * *=p<.001$ 


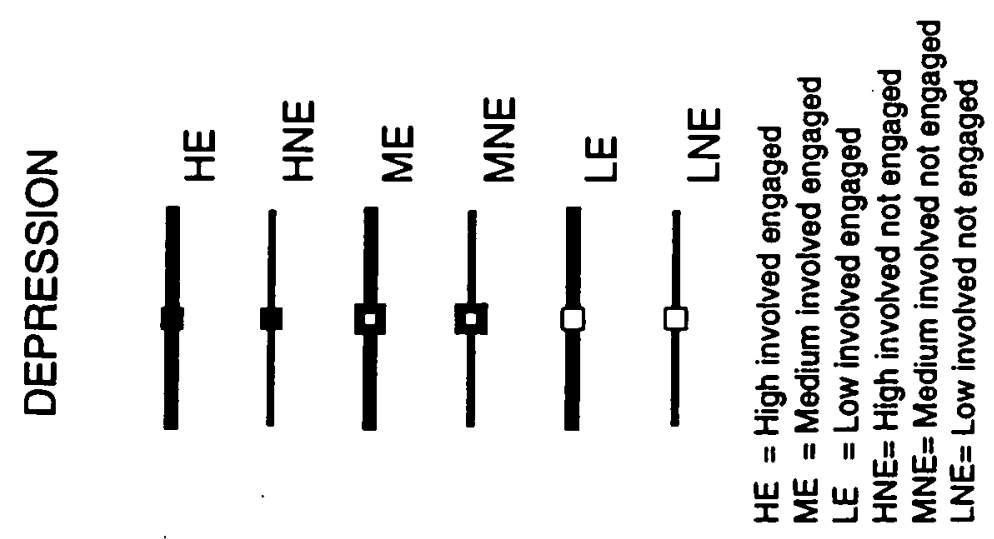

$\stackrel{\infty}{-\oplus}$
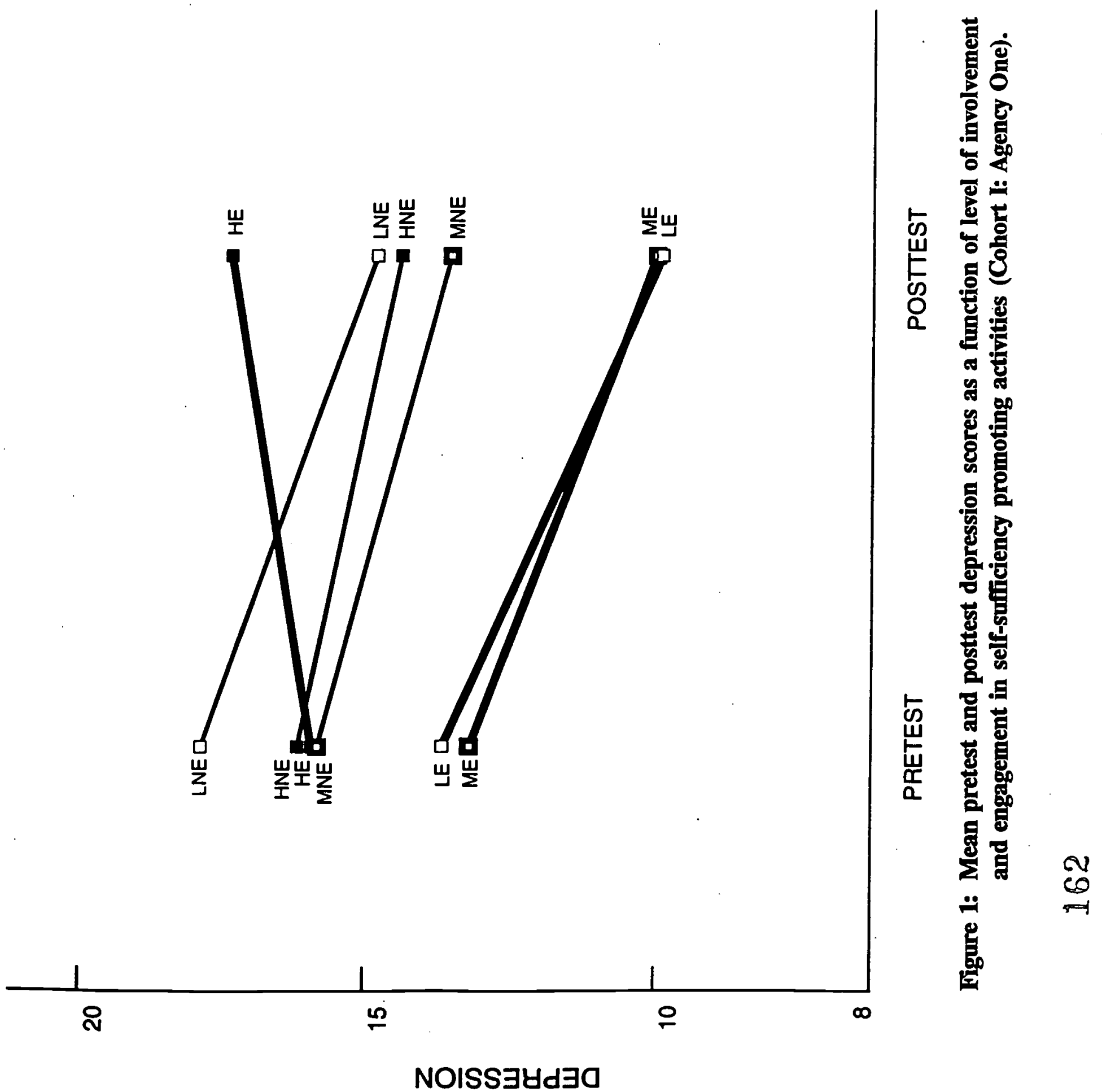


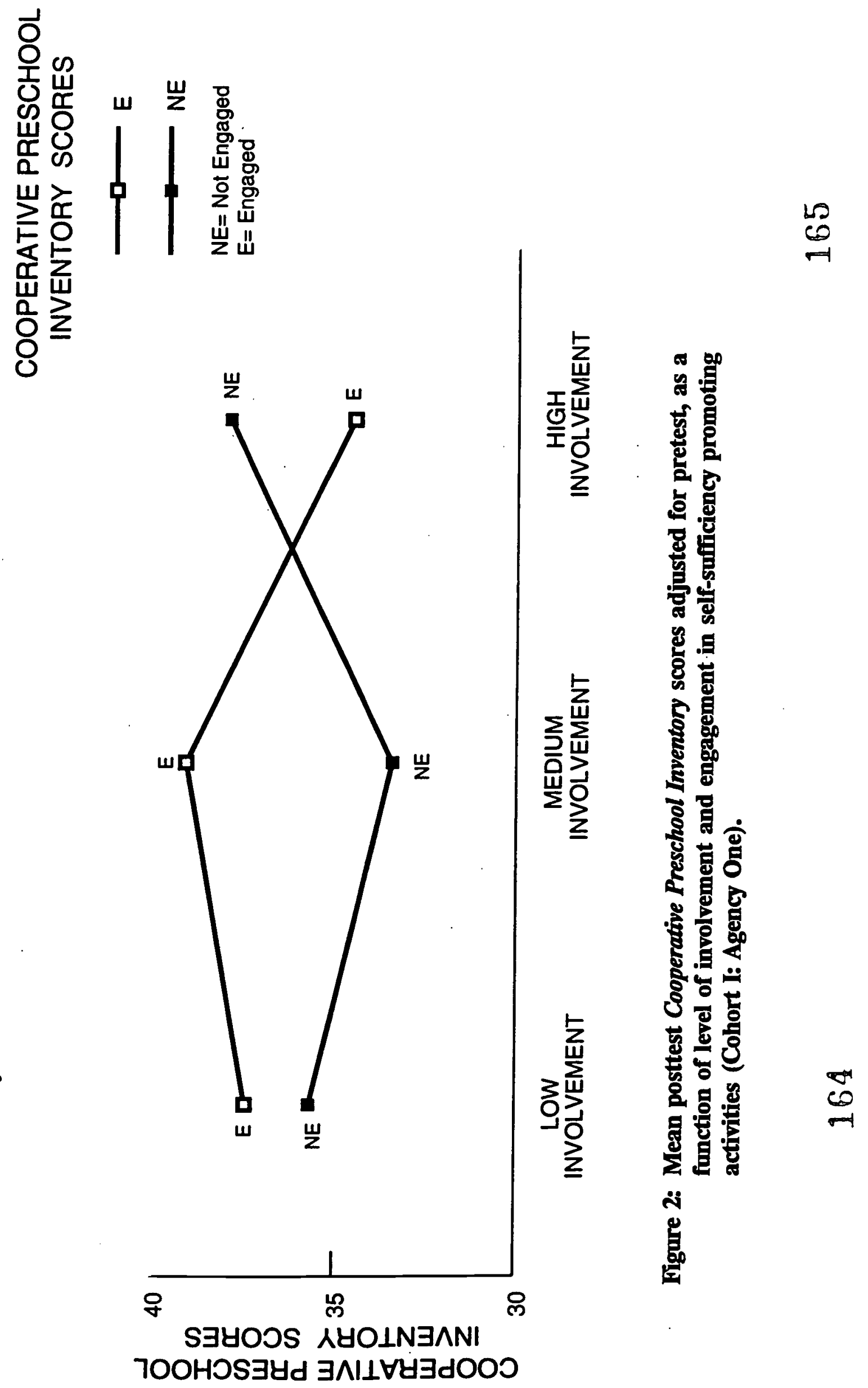



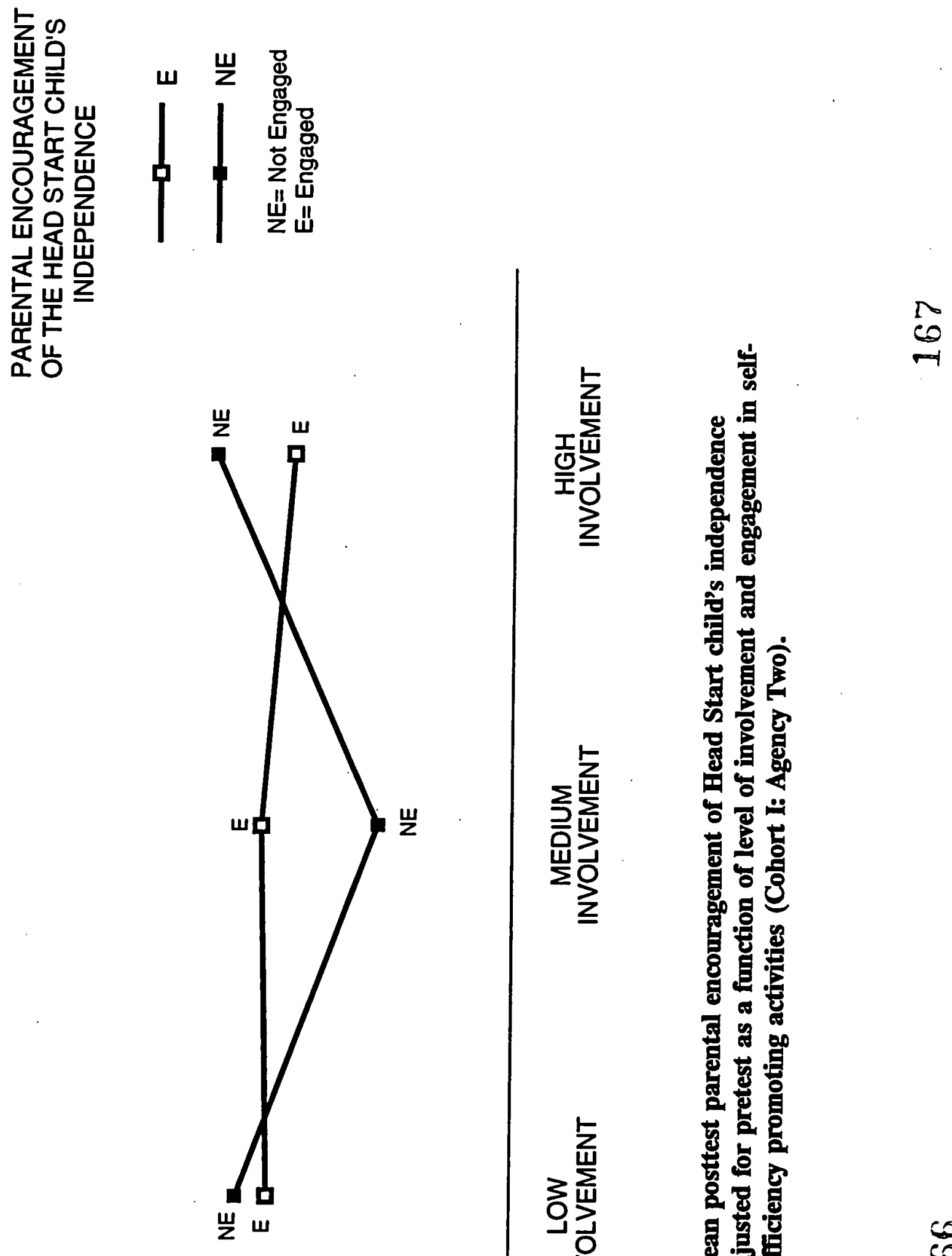


\title{
EXECUTIVE SUMMARY
}

\section{- FINAL REPORT \\ PARENT INVOLVEMENT IN HEAD START}

\author{
Authors: \\ Faith Lamb Parker \\ Chaya S. Piotrkowski \\ Susan Kessler-Sklar \\ Amy J. L. Baker \\ Lenore Peay \\ Beryl Clark
}

Prepared by:

- NCJW Center for the Child a

In Collaboration With:

The Bush Center in Child Development and Social Policy, Yale University

Ft. George Community Enrichment Center, Inc.

Staten Island Mental Health Services Head Start

30

$\triangle 2$

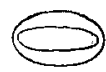

(3)

(6)

$\infty$

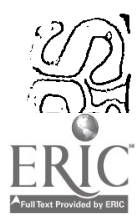

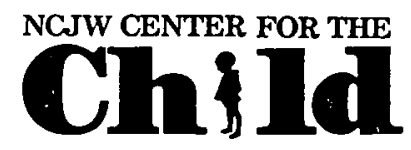

53 West 23rd Street - New York • NY 10010

TEL: (212) 645-4048 • FAX: (212) 645-7466

(C) National Council of Jewish Women, 1997. All rights reserved. 


\section{NOTE:}

Faith Lamb Parker, Ph.D., formerly Senior Research Associate/Associate Director, NCJW Center for the Child is currently Assistant Clinical Professor, School of Public Health, Columbia University [TEL: 212-304-5251; FAX: 212-544-1911; e-mail: flp1@columbia.edu]. Chaya S. Piotrkowski, Ph.D., formerly Director, NCJW Center for the Child is currently Professor, Graduate School of Social Services, Fordham University [TEL: 212-636-6652; FAX: 212-666-3658]. Susan Kessler-Sklar, Ph.D. is Senior Research Associate, NCJW Center for the Child [TEL: 212-645-4048; FAX: 212-646-7466]. Amy J. L. Baker, Ph.D., formerly Senior Research Associate/Associate Director, NCJW Center for the Child is currently Director, NCJW Center for the Child [TEL: 212-645-4048; FAX: 212-645-7466]. Lenore Peay, B.A. is Director, Ft. Geonge Community Enrichment Center, Inc. [TEL: 212-927-2210; FAX: 212-740-5540]. Beryl Clark, M.S. is Executive Director, Staten Island Mental Health Services Head Start [TEL: 718-987-7755; FAX: 718- 987-2909]. 
- Major Findings from the Head Start Parent Involvement Project

\section{HOW DOES HEAD START PARENT INVOUVEMENT \\ IMPROVE THE LIVES OF FAMIIIES?}

Through An Improved Parent child Relationship?

4. 1.ess parentat strithess.

- I.ess parenting agstovation.

Through An Enhanced Home Learning Environument:

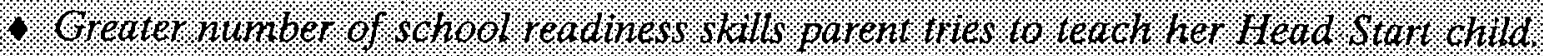

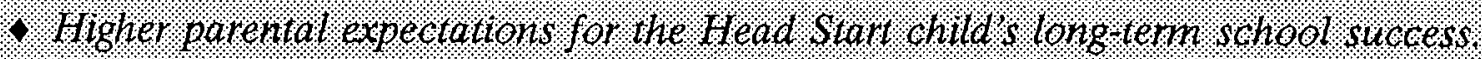

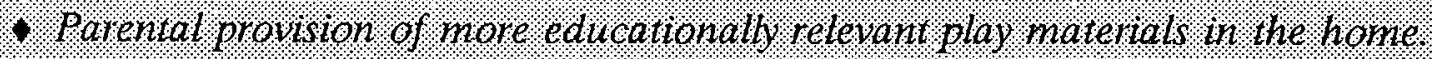

Through Chillaren's Greater Social Competence:

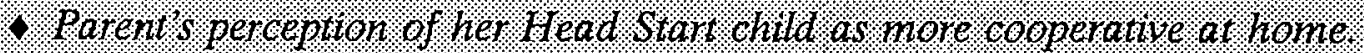

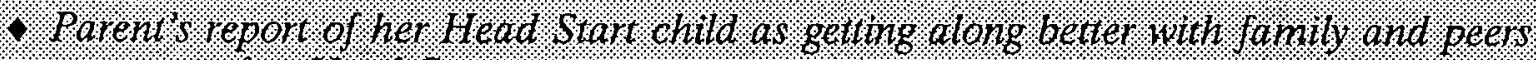
one jear after Hieat Start.

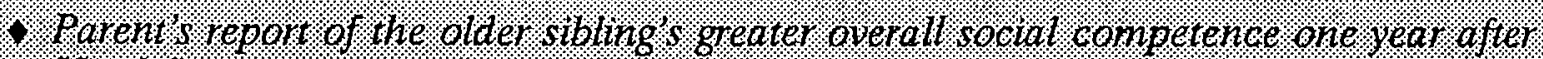
Head Stan.

Through Greater Parent Involvemeat in Glementart. School:

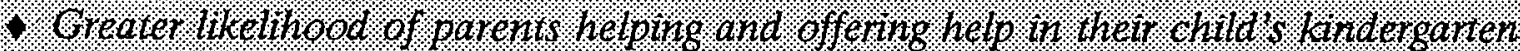
classtoon

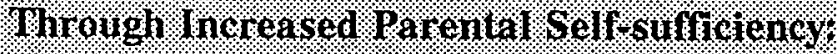

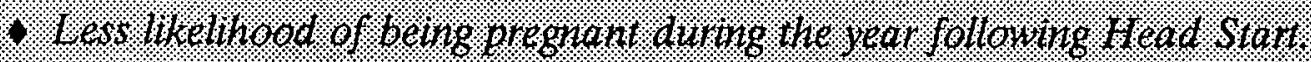

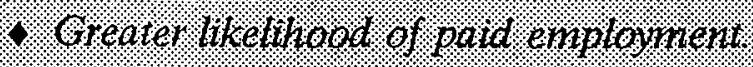




\section{WHO GETS INVOLVED IN MEAD START?}

\section{Parents Who:}

? Reported heeding edication, 106 irathing, or patd employnent al the begithing of the thead Start year.

8. Were not pregrant dinng the Head Stan year.

6. Were not engaged in self suffictency promoling activities ledication, lob training employnent dung the Head Stant vear.

4 Reported less frequent parenting hasstes:

4 Reported less intense parenting hosstes.

4 Had greater expectalions for thet Head Start chlld longterm scheol success:

4. Had a jounger Head Stat chith.

- Had a head Stent child with fewer bith difficutties

5. Hid a Head Start chlld With greater healih problems.

9. Reported the older sibling to have betrer school performantee 


\section{WHO UTILITES WEAD START SOCLAL SERVICES?}

\section{Demographic Cactors}

Parents 1720 .

1. Were older.

1. Were less educated.

1. Reported nore self suffletenor needs.

\section{Barent.Child Relationship and Homed Gearing Envionment:}

Parents 17.0.

100.01010101

4 Spoke nore frequenty hith theit chitd 0 bout school.

4 Helped heil ghid leann nore school readiness shils.

\section{Head Stain Gild and Older. Siblings:}

Porents 1710 .

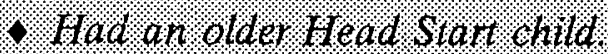

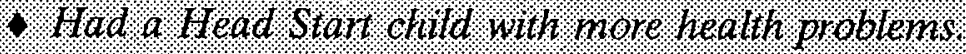

5. Hlad on t older' older sibting.

$\checkmark$ Reported the older sibling as having poorer school performance.

4. Reported the older sibling to have better peer and forith relations. 


\section{POLICT AND PROCRAM RECOMMEND TTIONS}

4. Reexamine the needs and shills of parents and how hedd Stant can address these through the ypes of involvement activites offered.

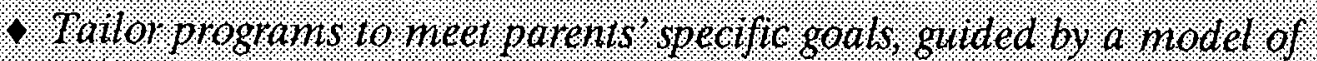
which aspects of patent involiement have which outcomes.

$\checkmark$ Redefine Head Start parent involvement lo include achivities initiated by.

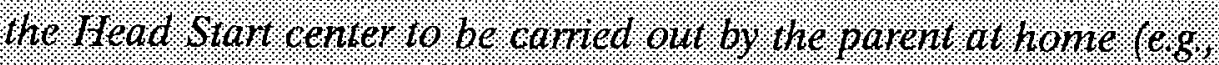

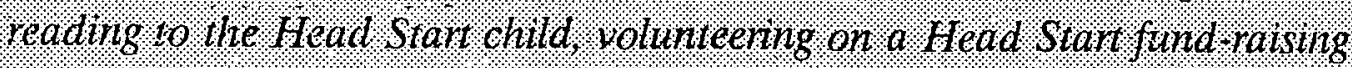
activity):

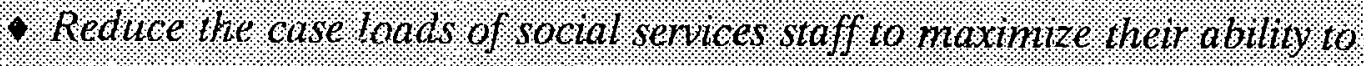

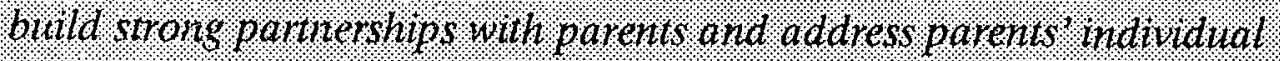
needts so that thet cont attath their goats.

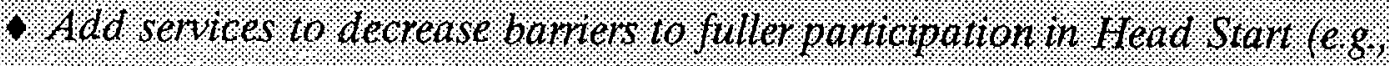

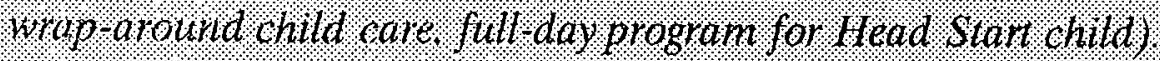

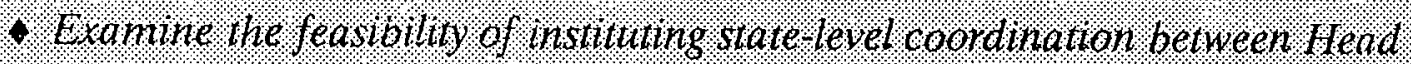
Stant agencies and welfare to wort programs so that Hed d Start agencles

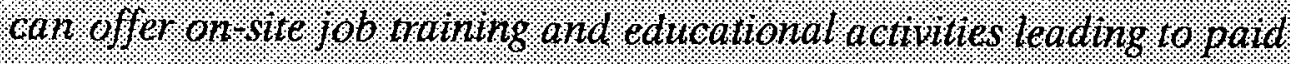
employment as part of their parent intolvenent component. 


\section{ACKNOWLEDGMENTS}

There are many people whom we would like to thank for their help and expertise in conducting this complex research project. First we would like to thank the Head Start Research Group (HSRG) for their hard work, creativity, and support in developing and implementing the three parts of the Parent Involvement Project: the parent involvement survey; the longitudinal study; and the staff development and training intervention. The original group included Blanca Baez, Clare Marie Bonafede, Jane Bradshaw, Beryl Clark, Margaret Dana, Elaine Danavall Williams, Catherine Faye, Pamela Freeman, Margaret Hernandez, Rochelle Moody, Lenore Peay, John Van Orden, Ruth Robinson, Kiley Roman, Aida Seda, Susan Young and Dr. Edward Zigler. We also would like to thank those who joined the HSRG later in the project, for their assistance.

Next, we would like to thank the parents and children from the two Head Start agencies who participated in the project. Confidentiality precludes naming them personally, but we gratefully acknowledge their contribution.

Dr. Edward Zigler and Elaine Danavall Williams deserve special mention. As consultants to the HSRG, they freely offered their time and expertise over the course of the project. We are also grateful to Dr. Louis Primavera and Dr. Bernard Gorman for their statistical talents and analytic skills in helping to bring the longitudinal study to completion.

Cynthia Flynn and Hirokazu Yoshikawa are given special thanks. Cynthia, for her undaunted energy and extraordinary interpersonal ability in carrying out the objectives of the project, and Hiro, for his meticulous and enthusiastic participant in the parent involvement survey.

Chabiram Rampersad's excellent skills, loyalty, and steadfastness in his administrative and fiscal roles throughout the life of the project has been invaluable to its successful completion. Acknowledging him, and his assistant Nuris Cevallos, for providing administrative support and for producing numerous drafts of the manuscript, including the final report and this executive summary, is especially important.

We also would like to thank Dr. Laura Soden and Jennifer Ritter for their efforts in cleaning and verifying the voluminous amounts of data, gathering information and contributing to the initial drafts of the manuscipts.

Finally, we must express our deepest gratitude to the Administration on Children, Youth and Families and the U.S. Department of Health and Human Services for its support of this enormous project. In particular, the Federal Project Officers Adrienne Brigmon and Dr. Esther Kresh are thanked for their support and considerable patience over the years.

Without the efforts, contributions, talents and support of all of the above-mentioned, this project would not have been possible. 


\section{INTRODUCTION}

Head Start is a comprehensive, federally funded program for low-income families with young children. Since its inception during President Johnson's War on Poverty, Head Start has served as a model for the delivery of comprehensive child development services. Today, Head Start stands as the first two-generation intervention designed to focus on children's development and well-being within the context of strong family support and involvement. Head Start seeks to alleviate family poverty and increase the sense of dignity and self-worth of family members (Parker, Piotrkowski, Horn, \& Greene, 1995). From its inception, Head Start's legislative mandate called for "maximum feasible participation" of parents in all programmatic efforts and policy decisions. Parental involvement has always been an integral component of the Head Start program model [U.S. Department of Health and Human Services (USDHHS), 1970].

Traditionally, research on Head Start's effectiveness has focused on its impact on the Head Start child; specifically, on his or her cognitive, language, and motor development, and health, nutrition, and subsequent educational attainment. Policymakers and practitioners alike have used this child-focused research base as their sole source of evidence for assessing the efficacy of the program, despite the fact that Head Start founders -- notably Dr. Edward Zigler -- see parents as the vital force behind long-lasting effects of Head Start on families. Dr. Zigler has consistently called for research that focuses on parents in addition to children (Zigler, 1978; Zigler \& Muenchow, 1992; Zigler \& Styfco, 1993; Zigler \& Valentine, 1979). Furthermore, legislated federal panels and focus groups, as well as the National Head Start Association, have strongly advocated for research that explores parent involvement in Head Start (NHSA, 1990; USDHHS, 1993; NAS, 1996). Nevertheless, there is little research on the benefits of Head Start to parents and on the role of parents as mediators of child and family outcomes. This lack of attention to parents is unfortunate because only by investigating the potential benefits of parent participation for parents themselves and for other family members can the full impact of Head Start be understood (Parker, Piotrkowski \& Peay, 1987; Slaughter, Lindsey, Nakagawa, \& Kuehne, 1989). In light of President Clinton's pledge in his first State of the Union address of 1997 to expand Head Start by 2002, documenting all of the benefits of Head Start to justify the investment of these substantial federal funds becomes more crucial than ever before.

The National Council of Jewish Women (NCJW) Center for the Child, in collaboration with the Bush Center in Child Development and Social Policy at Yale University, undertook the Head Start Parent Involvement Project in 1990 to explore this virtually untapped area. This five-year initiative consisted of three parts:

- A parent survey exploring life events and experiences that might function as barriers to parents' participation in Head Start;

- A longitudinal study of parent involvement in Head Start assessing its impact on parents, Head Start children, and their siblings;

- An intervention focused on staff development and training to enhance staff's work with Head Start parents. 
The first three years of this project were funded through a Head Start/University Partnership Grant from the Administration on Children, Youth, and Families (ACYF) and NCJW. Later funding from NCJW and private foundations helped support the project and enabled a follow-up study of Head Start children and their families in elementary school.

The project was designed and implemented through a partnership of NCJW Center for the Child and the Head Start community. The commitment to this joint effort was based in the belief that both practitioners and researchers have expertise and creativity that, when combined, improve the validity and utility of the results. The partnership, named the "Head Start Research Group" (HSRG), was comprised of representative Head Start staff and parents, Center for the Child research staff, and consultants from the research and the practitioner communities. The HSRG worked together throughout the project to refine the designs, constructs, and methods of the studies; to develop and/or select measures; and to implement all phases of the project.

\section{The Participating Head Start Agencies}

Two Head Start delegate agencies in New York City participated in the project. Agency One was established in 1981. with Head Start expansion money; Agency Two was one of the original Head Starts, established in 1966. Both agencies use a standard center-based model with half-day and full-day classrooms. While Agency One's two centers are within walking distance in the same community, Agency Two has one center that buses families from three widely dispersed geographic areas to a middle-class neighborhood. Agency One serves Latino families of Dominican origin (99.6\%), while Agency Two's families are predominantly African American. Virtually all of the families from both agencies have incomes below the poverty line and receive Temporary Assistance to Needy Families (TANF) benefits.

Based on community needs assessments, police reports, and other sources of demographic data, ${ }^{1}$ the following information about the catchment areas of the two participating agencies at the time of the study was available.

The catchment area of Agency One is a recently immigrated Latino (mostly Dominican) community in which half the residents speak little or no English. Forty-four percent of the Dominican homes are headed by women. The high school drop out rate in the community for Dominicans is 66\%. The neighborhood surrounding Agency One is reported to be among the most dangerous in the city. Related to the high homicide rate is the pervasive and violent underground drug economy. Nevertheless, the community lacks adequate police protection, with only one precinct serving the area.

Agency Two's catchment area is large and diverse in terms of ethnicity and SES, with African-American, Asian, Euro American, Middle-Eastern, Native-American, and Latino ethnic groups represented. The majority culture is English speaking (89\%), Euro-American $(90 \%)$, native born $(88 \%)$, and with wages or salary as the source of income $(81 \%)$.

Educational attainment in this middle-class community is relatively high, with almost threefourths of adults $(72 \%)$ completing their schooling. The crime rate appears to be moderate, compared to statistics for the city as a whole. Within this emerging picture of a nearly homogeneous, White, middle-class borough, pockets of poverty coexist.

Families and staff at these two Head Start agencies participated in all three parts of the Head Start Parent Involvement Project. 
PART I: A PARENT SURVEY: LIFE EVENTS AND EXPERIENCES AS POTENTIAL BARRIERS TO PARENT INVOLVEMENT

Faith Lamb Parker, Chaya S. Piotrkowski, Lenore Peay, Beryl Clark, Hirokazu Yoshikawa, Susan Kessler-Sklar, \& Amy J. L. Baker.

\begin{abstract}
Sixty-eight mothers differing in level of Head Start involvement as judged by staff were surveyed about the occurrence of 33 life events and experiences during the Head Start year and their perceptions of these events as barriers to fuller involvement. Twenty-two of the life events were reported by at least $10 \%$ of the mothers. Eight of the life events were associated with reduced involvement, whereas two were associated with increased involvement. Mothers' expressed a need for additional job-training activities as part of their Head Start experience.
\end{abstract}

\title{
INTRODUCTION AND METHODS
}

A survey was developed to generate information about the types of life events experienced by Head Start mothers and the extent to which they perceived these life events as barriers to their involvement in Head Start. The number and types of life events experienced were also related to Head Start staff's ratings of the mothers' level of involvement compared to other mothers in the program.

The Barriers to Head Start Parent Participation Survey was conducted through personal interviews in June 1991. The survey explored indepth four domains of life events and experiences that represent potential barriers to mothers' participation in Head Start activities and experiences: 1) institutional -- quality and types of activities offered, appearance of physical space, perceptions of staff friendliness; 2) personal -- race and ethnicity, job training and education, health, and psychological well-being; 3 ) family and friends -- household size, ages of children, and supports and drains in and outside of the household; and 4) community -- neighborhood safety, accessibility of transportation, and availability of child care.

The HSRG's decision to conduct the survey was based on interest in and concern about why some parents were less involved in parental experiences and activities at these agencies. Survey items were selected by members of the HSRG based on a review of the literature and their experience with Head Start families. A summary section of the survey listed 33 life event items; mothers indicated whether they had experienced each of the 33 events and if so whether or not they perceived that event as a barrier to their involvement. The mothers also answered two open-ended questions about different ways 
that parents could be involved in Head Start and their ideas about how to improve the parent involvement component.

Each Head Start mother at the two agencies was classified by staff as a low, moderate, or high participator. Research staff randomly selected 72 mothers, 24 from each of the three involvement categories (low, moderate, high). Sixty-eight of the $72(94 \%)$ invited mothers participated in the survey.

\section{HIGHLIGHTS OF RESULTS AND DISCUSSION}

\section{What Life Events Are Experienced by Head Start Mothers?}

A mixture of personal, family, and community life events was reported by the mothers. The most frequent of these was "often feeling sad, down, depressed" during the Head Start year $(47 \%)$. Furthermore, about one-quarter $(27 \%)$ reported that they "lacked energy or had little interest in things." Having a major health problem also ranked among the personal factors mentioned by the mothers (21\%). Parental factors ranking high on the list included having a baby or toddler at home (40\%), having children with a disability or behavioral problem $(29 \%)$, and not having dependable, convenient child care $(21 \%)$. Factors related to maintaining a home also were prevalent in these women's lives. Mothers reported lacking heat, hot water, or electricity (34\%), experiencing flood, fire, or other disaster (21\%), and having no telephone (19\%) during the Head Start year. That nearly half of the mothers experienced depressed feelings and one-fifth to onequarter reported having a major health problem, a child with a disability or behavior problem, no dependable child care, or a combination of these speaks to the immense burden of living in poverty.

Several life events related to self-sufficiency (i.e., job training, education, employment) were ranked among the top 15: working, going to school, or volunteering outside Head Start (44\%), having a schedule that conflicted with : Head Start activities (37\%), and having inflexible hours at work or school (26\%). Interestingly, the only barrier mentioned that related to the Head Start agency or program itself was not being satisfied with the physical space allocated for parent involvement activities (19\%).

\section{Are These Life Events and Experiences Related to Level of Involvement?}

Mothers varied greatly in the total number of events experienced, with the average number between five and six. Of primary interest to this study was the extent to which these events functioned as barriers to mothers' involvement. We addressed this question in two ways. First, we asked whether number of events was related to level of involvement. Results revealed that mothers reporting more life events and experiences were rated by staff as significantly less involved in Head Start. 
We also examined whether each event alone was associated with level of involvement. These analyses focused on the 22 events that were reported by at least $10 \%$ of the mothers. Eight of these 22 life events were associated with less participation, including feeling sad, down, or depressed, having a conflicting schedule, working or going to school during the day, having a baby or toddler at home, having a child with health problems, moving during the Head Start year, and experiencing a flood, fire, or other disaster. Two demographic variables were found to be associated with less staff-judged involvement: 1) having more adults in the home and 2) being married, suggesting that activities, chores, and possibly the attitudes of others in the home may be important factors influencing mothers' level of participation in Head Start. Two life events were associated with greater involvement: feeling shy and being without heat, hot water, or electricity. The direction of these two relationships suggested that mothers may utilize Head Start as a supportive institution and as a temporary "home away from home" when necessary.

\section{Do Mothers Perceive These Life Events as Barriers to Their Participation in Head Start?}

Of the 22 life events experienced by at least $10 \%$ of the mothers, 20 were perceived as barriers to involvement by at least one mother. There was considerable variability in the percentage of mothers perceiving each of the 22 life events and experiences as barriers to their participation. The most frequently perceived barrier, among those who indicated that the event applied to them, was having a schedule that conflicted with Head Start activities (84\%). Interestingly, although almost half of the mothers reported often feeling sad, down and even depressed, only one-third believed that those feelings hindered their participation. Thus, mothers do not perceive their depressive feelings as interfering with their ability to be involved in Head Start. A somewhat different picture emerged, however, from staff reports. Head Start staff perceived the mothers who characterized themselves as depressed as less involved. Such differences in perception of level of involvement between staff and parents should be of interest to Head Start administrators, because staff and parents must work together to address parents' needs and goals. Developing shared goals and perceptions is an important ingredient in addressing barriers to the development of an effective parent involvement program (see Part III).

\section{How Can Parents be Involved in Head Start?}

Mothers' ideas for how they could be involved in Head Start were consistent with the Head Start program performance standards (USDHHS, 1996): 1) direct involvement in decision making in program planning and operations; 2) participation in classroom and other program activities as paid employees, volunteers, or observers; 3 ) activities for parents that they have helped to develop; and 4) working with their children in 
cooperation with Head Start staff. Some mothers also mentioned activities related to other parents or staff, such as "socializing" or "meeting new parents" and having supportive relations with staff. These experiences, although not explicit in the standards, reflect the spirit that permeates Head Start for some parents and may in fact be critical to its long-term success.

\section{How Can the Parent Involvement Component be Improved?}

The suggestion most frequently made by mothers for improving Head Start parent involvement was to have more self-sufficiency related activities such as ESL, GED, and other skill-building training experiences. Anecdotal evidence (e.g., Replogle, 1995) also supports these findings. Head Start and TANF program developers and policy makers should take these data into account as they consider how Head Start parent involvement might be effectively integrated into present welfare-to-work efforts. Many current programs requiring TANF-supported parents to participate in job training and education (Family Support Act of 1988) create conflicts for parents and for staff, who are trying to recruit and engage parents required by law to be involved elsewhere. Recent thinking on this issue (Herr, Halpern \& Majeske, 1995; Parker et al., 1995) has urged policymakers at both federal and state levels to coordinate with each other and to integrate Head Start's parent component with the welfare reform agenda to reduce duplication of services and to help parents build skills that could lead to economic selfsufficiency. Coordinating welfare-to-work programs with new or expanded Head Start programming in this area would enhance the value of Head Start to its communities, a goal of the program that was central to its original mandate.

\section{Summary}

The results of this survey provide insight into the daily lives of Head Start mothers and suggest several avenues for enhancing staff-parent relationships and for integrating Head Start with the current welfare reform legislation. The Head Start mothers who completed this survey revealed that they experienced a variety of stressors and major life events during the Head Start year. Interestingly, mothers did not always perceive these life events to be barriers to their involvement. They did report that having a work schedule that conflicted with the Head Start schedule was a barrier to fuller participation. Mothers who reported a greater number of total life events were rated by staff as less involved compared to other mothers. The mothers also shared their ideas for improving the parent involvement component of Head Start, focusing specifically on opportunities for enhancing their self-sufficiency through ESL, GED, and other skillbuilding and training experiences. 


\section{PART II: THE LONGITUDINAL STUDY: THE IMPACT OF PARENT INVOLVEMENT ON PARENTS AND CHILDREN}

Faith Lamb Parker, Chaya S. Piotrkowski, Susan Kessler-Sklar, Lenore Peay, Beryl Clark, Laura M. Soden, \& Amy J.'L. Baker.

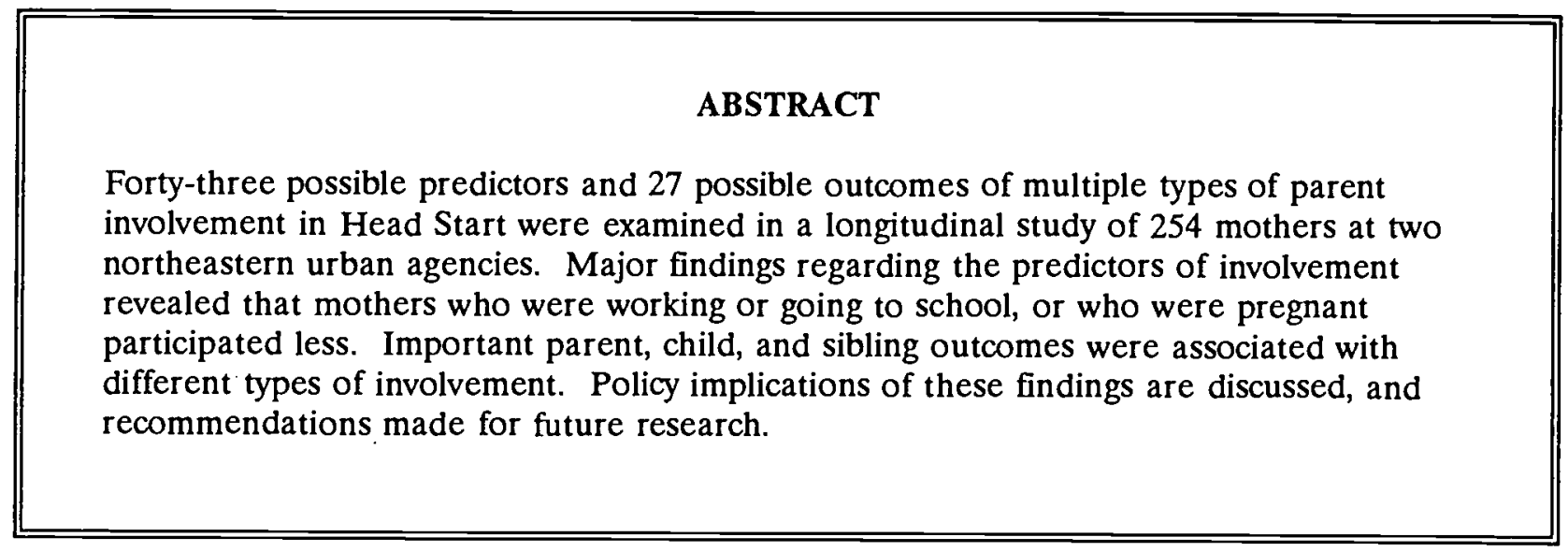

\section{INTRODUCTION}

The present study of parent involvement was initiated because of a clear need to rigorously understand parents' participation in Head Start. It builds on previous work (Oyemade, Washington \& Gullo, 1989; Parker et al., 1987; Slaughter, Lindsey, Nakagawa, \& Kuehne, 1989) and extends it by: 1) using pretest measures of outcomes to control for initial differences; 2 ) using multiple objective measures of parent involvement; 3 ) assessing the impact on a wide array of outcomes; 4) employing a longitudinal design that follows the Head Start family one year after the end of the program; and 5) assessing the impact of parent involvement on an older sibling.

This project also moves the field forward by attempting to further understand what characteristics and circumstances of parents and children predict parent involvement. This study examines the effect of a wide range of predictor variables, including demographic, personal, and contextual characteristics of the parent, the Head Start child, and older siblings, on multiple types of involvement.

Parent involvement in Head Start is the major independent variable in this study. We hypothesized that more involvement would be related to better outcomes for parents and children. Building on the variety of ways parent involvement has been conceptualized (Parker et al., 1987; Slaughter et al., 1989; Valentine \& Stark, 1979), two ways of conceptualizing involvement were identified for this study. First, parent involvement was conceptualized as the amount of parent activities and experiences in the Head Start program. Second, the HSRG identified different types of activities parents could 
participate in, based on the Head Start Performance Standards: 1) volunteering in the classroom, office, and kitchen and on field trips; 2) attending workshops on parenting, child development, and nutrition; 3) participating in policy-making committees and meetings, including such activities as class, agency-level, and personnel practices committee meetings; and 4) utilizing social services, including contacts about personal problems; the Head Start child and/or another family member, housing, financial, or immigration issues, and community resource referrals.

Social competence is the major outcome domain for Head Start parents, Head Start children, and their older siblings (Zigler and Trickett, 1978), defined as: 1) successfully meeting society's expectations and 2) personal development and self-actualization. This study examines the impact of parent involvement in Head Start on the social competence of parents, Head Start children, and their siblings.

Parent involvement in Head Start might enhance parents' social competence by providing them with support and services to reduce life stress, by enhancing skills that promote the confidence to try new behaviors, and by facilitating parents' feelings of efficacy and control over their own lives. Because the concept of social competence is broad and multifaceted, we focused on those aspects of social competence that seemed likely to be affected by parent involvement in Head Start. Most of the outcomes were assessed at the end of the Head Start year. Those that were assessed at the end of the following year are noted as such. Specific aspects of parental social competence assessed as outcomes in this study include:

$\diamond$ Positive parent-child relationship: parental encouragement of the child's independence, lower parental strictness and aggravation, less frequent daily parenting hassles;

\& Enhanced home learning environment: higher parental expectations for the child's long-term school success, parental report of teaching the child more school readiness skills, and the provision of educational learning materials in the home;

$\diamond$ Greater involvement in the Head Start child's education in kindergarten: ratings of overall involvement in kindergarten, a parent's helping or offering to help in the classroom;

- Skills and behaviors that promote socioeconomic self-sufficiency: paid employment during the year following Head Start, participation in education or job-training activities, not becoming pregnant during the year following Head Start;

- Enhanced subjective well-being: feelings of mastery and control and lack of depressive symptomatology. 
Children's social competence might also be enhanced through parents' involvement in Head Start. Specifically, the parent-child relationship might be affected by increased feelings of parenting competence, new understandings of child development, and improved methods of interaction between parent and child. In addition, parent involvement could enhance how parents deal with other social institutions, such as schools. Thus, parents' involvement in all aspects of Head Start might have benefits for the Head Start child as well (in addition to the direct benefits Head Start children receive from their center-based experience). Specific aspects of children's social competence assessed as outcomes in this study include:

- Greater school readiness and social competence: school readiness, greater cooperativeness, and less disruptiveness at home;

- Greater elementary school adaptation and social competence: teacher ratings of adaptation to the kindergarten classroom, teacher ratings of higher current and anticipated school performance, parent's report of the child's positive relationships with other family members and peers, parent's report of the child's greater ability to work and play alone.

Finally, parent involvement in Head Start might enhance the social competence of the older siblings of the Head Start child. The older siblings might benefit from potential changes in their parents' child-rearing attitudes and feelings of personal well-being as

well as from changes in the home learning environment. ${ }^{2}$ Specific older sibling outcomes include:

- Greater social adjustment one year after Head Start: parent's report of sibling's overall social competence, sibling's community activities, relationships with family and peers, and school grades.

Therefore, the objectives of the study were: 1 ) to identify the "predictor" (i.e., demographic, contextual, and personal) variables associated with parents' participation in Head Start, and 2) to examine the relationship between parent participation in Head Start and positive outcomes for the parents themselves, the Head Start children, and siblings.

\section{METHOD}

\section{Design and Data Collection Procedures}

The design for the longitudinal study is correlational with pretest and posttest data collections (Cook \& Campbell, 1979). Two hundred and three Agency One mothers and their families and 103 Agency Two mothers and their families responded to invitations to participate. In the fall of 1991, the mothers completed a series of measures and Head 
Start staff assessed the children. Data were collected on parent and child outcomes at the end of the Head Start year. One year later a telephone interview was conducted with the mothers. At the same time, data were collected from the children's teachers on their school functioning and on the mothers' involvement in the elementary schools. The attrition rate was average for research with this population.

\section{Subjects}

Two-hundred and three mothers and their Head Start children out of a possible 256 at Agency One (79\%) and 103 mothers and their Head Start children out of a possible 151 at Agency Two (68\%) agreed to participate and were pretested. Ninety-eight percent of the Agency One families were Dominican and $83 \%$ were born outside the United States, with Spanish as their dominant language. Agency One mothers' ages ranged from 21 to 62 years, averaging 34 years of age. Forty-two percent of the mothers did not finish high school. One hundred and thirty two of the Head Start children were four year olds with no previous Head Start experience. One school-aged sibling between the ages of 5 and 12 was selected from each Agency One family participating in the study and having a sibling within that age range balancing for age and gender. Eighty-six siblings were included. Of the 203 Agency One families, 172 have complete data sets and therefore constitute the analyzed sample.

One-hundred and fifteen Agency One families with older children entering kindergarten in September 1992 comprised the follow-up sample. Forty-four siblings were included in this follow-up.

The 103 pretested families at Agency Two were English speaking (90\%) and primarily American-born and educated. Their diverse ethnic backgrounds included African American (60\%), Hispanic (18\%), and Euro American (18\%). Mothers ranged in age from 18 to 54 (average age was 31 years). Sixty-four percent had completed high school. Fifty-seven of the Head Start children were four year olds with no previous Head Start experience. Eighty-two of the families have complete data sets and therefore constitute the analyzed sample.

\section{Measures and Variables}

The measures used to assess the major constructs of the study fall into three major categories: measures of predictor variables, measures of parent involvement, and measures of outcome variables. Table 1 summarizes the measures used and the sources of the data. The parent involvement variables are detailed below.

Four variables measuring parent involvement in Head Start were used. The first was a continuous variable of overall involvement ${ }^{3}$ representing the number of hours the parent 
was at the center during the Head Start year. This variable did not include the number of hours parents utilized social services because utilization of social services was qualitatively different from the other parent involvement activities. ${ }^{4}$

Two parent involvement "activity type" variables were created based on preliminary examinations of the parent involvement data: volunteering and attending workshops/policy meetings. ${ }^{5}$ These variables represented not just amount of involvement but type of activity as well. A fourth variable represented the number of hours parents spent utilizing social services.

Because the parent involvement component at each site was defined and implemented differently in response to the cultural backgrounds of the families, all data were analyzed separately by agency.

\section{RESULTS AND DISCUSSION}

\section{Who Gets Involved in Head Start?}

The associations among 43 demographic, maternal, family, and contextual factors and the four types of parent involvement were assessed. The predictors of volunteering, workshops/policy meetings, and overall involvement are presented first, followed by the predictors of utilization of social services. In addition to statistically significant associations, trends are included because they represent meaningful effect sizes (Cohen, 1977). Mothers who became more involved at Agency One include:

- mothers who reported needing education, job training, or paid employment at the beginning of the Head Start year (overall involvement, volunteering, workshops/policy meetings);

- mothers who were not pregnant during the Head Start year (overall involvement, volunteering, workshops/policy meetings);

- mothers who were not engaged in self-sufficiency-promoting activities (education, job training, employment) during the Head Start year (overall involvement, volunteering, workshops/policy meetings);

- mothers who reported less frequent parenting hassles (overall involvement, volunteering);

- mothers who reported less intense parenting hassles (overall involvement, volunteering);

- mothers with a younger Head Start child (overall involvement); 
- mothers whose Head Start child had experienced fewer birth difficulties (volunteering);

- mothers whose Head Start child experienced greater health problems (volunteering);

- mothers who reported that the older sibling had greater school competence (overall involvement, workshops/policy meetings); and

- mothers who reported greater expectations for their Head Start child's long-term school performance (workshops/policy meetings).

At Agency Two, the following findings replicated those at Agency One:

- mothers who were not pregnant during the Head Start year (overall involvement, volunteering, workshops/policy meetings);

- mothers who reported that the older sibling had greater school competence (overall involvement, volunteering).

\section{Who Utilizes Social Services?}

At Agency One, results pertaining to utilization of social services included:

- older mothers;

- less educated mothers;

- mothers who reported more self-sufficiency needs;

- mothers who helped their child learn more school readiness skills;

- mothers of Head Start children with more health problems;

- mothers of older Head Start children;

- stricter mothers;

- mothers who spoke more frequently with their child about school;

- mothers having an "older" older sibling;

- mothers who reported that the older sibling had poorer school performance; and

- mothers who reported that the older sibling had better peer and family relations. 
Based on previous research and theory, a large range of predictors were selected for inclusion in this study, many of which were related to involvement. Most noteworthy is the finding that engagement in self-sufficiency-promoting activities -- working, going to school, or job training -- was associated with less involvement in Head Start. This relationship was found as well in our Barriers to Head Start Parent Participation Survey (see Part I).

The total amount of variability accounted for by the predictors of involvement was modest, suggesting that the next step in understanding which factors predict parent involvement in Head Start requires the development and testing of an expanded theoretical model specifying the most important influences on parents' participation and further exploring the nature of parent involvement itself. Factors that were not analyzed in the present study but that might be included in future work are the quality of the parents' Head Start experience, the characteristics and quality of the Head Start program, the educational needs of the Head Start child, and the skills and preferences of the parents.

\section{How Does Parent Involvement Improve the Lives of Families?}

The Head Start Parent Involvement component was designed to have multiple benefits for both Head Start children and their families. To this end, parent involvement in Head Start has had two key goals: to promote parents' personal development and selfsufficiency and to promote their involvement in their children's development and school readiness. Major findings pertaining to these outcomes of parent involvement for both agencies are reported and discussed below. In addition to statistically significant associations, trends are included because they represent meaningful effect sizes (Cohen, 1977).

Parent-Child Relationship and the Home Learning Environment

- Mothers who participated more (volunteering) were less aggravated with their children.

- Mothers who participated more (overall involvement, workshops/policy meetings) were less strict.

- Mothers who participated more (overall involvement, volunteering, workshops/policy meetings) tried to teach their Head Start child a greater number of school readiness skills.

- Mothers who participated more (volunteering) had higher parental expectations for the Head Start child's long-term school success. 
- Mothers who participated more (volunteering) provided more educationally relevant play materials in the home.

Greater involvement by the mothers in Head Start was associated with direct benefits for the mothers, in terms of an improved parent-child relationship. More involved parents reported more positive feelings towards their Head Start child including less aggravation and less strictness. Mothers' involvement in Head Start was also associated with their enhanced ability to provide an enriched home learning environment for their Head Start child. Mothers who participated more were more able to provide their children with the educational resources they needed to succeed in school. This included teaching their child more school readiness skills and providing more educationally relevant play materials in the home. Mothers who participated more also had higher expectations for their child's long-term success in school.

Findings pertaining to an improved parent-child relationship and home learning environment were more consistent in Agency One than in Agency Two. The smaller number of positive findings for Agency Two may result from lower levels of parent involvement overall, programmatic differences, a smaller sample size, pretest differences in the amount of stress the mothers experienced (Agency Two mothers reported more stressors and higher levels of perceived stress), and the different ethnic composition of its families. Like Head Start agencies across the country, these two agencies differed in several ways. Thus, further research is needed to understand how these factors influence the outcomes of involvement for parents and children.

\section{Parent Involvement in Elementary School}

- Mothers who participated more (workshops/policy meetings) were more likely to help and/or offer help in their child's kindergarten classroom.

The present study explored the impact of Head Start parent involvement on parents' involvement in public school. More attendance at workshops and policy meetings during the Head Start year was associated with mothers' helping more frequently and/or more frequently offering to help in the classroom. Overall involvement and volunteering were not related to involvement in public school, suggesting that other variables are important for understanding parents' patterns of elementary school involvement (such as parents' work obligations, parents' needs and interests, the needs of the child, the receptiveness of the school to parents' participation, and the nature of the opportunities for involvement offered by the school). Family-friendly employment policies also may play a role. Currently, parents are often limited by their employers' family leave policies.

Pending federal legislation that allows parents to leave work to attend parent-teacher conferences, special class presentations, and school-wide assemblies may pave the way for more parent-school interaction. 


\section{Parental Self-Sufficiency}

- Mothers who participated more were less likely to become pregnant during the year following Head Start.

- Mothers who participated more were more likely to have paid employment during the year following Head Start.

Mothers who participated more in Head Start were less likely to become pregnant and more likely to have paid employment in the year following Head Start. Thus, mothers who were more involved appeared to have more inner and external resources one year after the end of Head Start -- even after controlling for important demographic characteristics -- than mothers who participated less. The financial benefits of paid employment and the absence of the stress associated with pregnancy may benefit both children and parents, resulting in multiple payoffs for the entire family.

\section{Parent Well-Being}

Contrary to previous research (e.g., Parker et al., 1987), in the present study mothers' involvement in Head Start was not associated with increased maternal psychological wellbeing, particularly in a decrease in depressive symptoms or a heightened sense of personal control and mastery. Three factors may in part account for this: differences in the conceptualization and measurement of parent involvement,${ }^{6}$ lower levels of participation than in 1987 , and changes in the larger society negatively affecting the life circumstances of parents in the 1990s.

This third explanation is of particular importance to policymakers and is consistent with results of post hoc tests that examined the combined influences of involvement and engagement in self-sufficiency-promoting activities. Results indicated that mothers who were highly involved in Head Start and also engaged in self-sufficiency-promoting activities were more depressed than other highly involved mothers, suggesting that mothers may be overloaded and burned out when the need or wish to be involved in Head Start and to achieve self-sufficiency are simultaneous. There also was tentative post hoc evidence that children's school readiness may be adversely affected by the combination of high involvement and engagement in self-sufficiency activities. Thus, both Head Start program developers and legislators must consider how to integrate time and training requirements for low-income parents with young children, to reduce their stress and maximize their emotional well-being, while still achieving the desired goals for the family. Future research should include developing a model of the processes by which involvement and engagement in self-sufficiency promoting activities affect parents and children. 


\section{Head Start Child's Social Competence}

- Mothers who participated more (overall involvement, volunteering, workshops/policy meetings) perceived their Head Start child to be more cooperative.

- Mothers who participated more (overall involvement, workshops/policy meetings) reported their child to get along better wi:h family and friends one year after Head Start.

Greater cooperativeness at home was reported by mothers who volunteered more, attended more workshops and policy meetings, or participated more overall. In addition, one year after Head Start, mothers reported that their Head Start child got along better with family and friends. Thus, in addition to the already established direct benefits of Head Start for children (McKey et al., 1985; USDHHS, 1996), this study suggests potential indirect benefits for children of their mothers' involvement in Head Start, e.g., better interpersonal relationships. The findings are encouraging for several reasons. First, these data suggest that the benefits of Head Start extend to settings other than the Head Start center. Second, they suggest that the benefits of Head Start extend to child outcomes other than cognitive performance. Third, benefits of parents' involvement were found one year after the end of the program, suggesting that the effects of parent involvement extend beyond the end of the program.

We also found tentative evidence that the Head Start child's school readiness was affected by the combination of involvement and engagement in self-sufficiency-promoting activities. Children of highly involved mothers who also worked, went to school, or were in job training tended to have lower school readiness than children of highly involved mothers who were not engaged in self-sufficiency-promoting activities. Together with our findings on maternal depression, these findings suggest that total amount of parental activity may affect parents, and through the parents, their children.

\section{Sibling's Social Competence}

- Mothers who participated more (volunteering) reported that the Head Start child's older sibling became more socially competent.

The present study was the first to gather data exploring the impact of parent involvement on the Head Start child's older sibling. Even with a small sample, the results suggest that at least one type of parent involvement -- volunteering -- has a positive impact on the sibling's overall social competence. This study may have underestimated the full impact of involvement on siblings because the sample size was small and a full range of outcomes was not assessed. 


\section{Different Types of Involvement}

The present study also explored the differential impacts of the two types of parent involvement -- volunteering and attending workshops/policy meetings -- each of which contained different content, required different parental behaviors and interactions with others; and had different goals. Examination of the pattern of correlations revealed tentative evidence of differences in the impact of volunteering and workshops/policy meetings. Volunteering in the classroom was associated with greater help offered to the child in learning school readiness skills, whereas involvement in workshops/policy meetings was more often associated with more positive relationships of the Head Start child with the mother, other family members, and peers. The former finding suggests that volunteering provides parents with information about their child's skills and capabilities as well as with instructional techniques and knowledge of the types of skills that preschool children should be mastering. The impact of workshops/policy meetings on parenting attitudes is consistent with the parenting focus of many of the workshops.

Results also revealed that overall involvement appeared to account for more of the statistically significant relationships with outcomes than did either of the two activity types. This may be because there was more range and variability in overall involvement, resulting in greater power to detect statistically significant relationships. The nature of Head Start record keeping, which necessitated combining somewhat different activities, ${ }^{7}$ might have masked the unique effects of different types of involvement.

The next step in understanding the benefits of specific parent involvement activities requires the development of a theoretical model relating specific activities to specific outcomes. Testing such a model would require that Head Start staff and researchers work together to develop a record-k-eping system that would more fully detail individual parent involvement activities. The results of such efforts could provide information to policymakers and educators about how to maximize the effectiveness of the parent component of the Head Start experience.

\section{How Does Parental Utilization of Social Services Improve the Lives of Families?}

Greater utilization of social services was associated with one outcome: mothers reporting that the Head Start child works and plays alone better than other children at one-year follow-up. The benefits of social services may be underestimated in the present study for several reasons:

- mothers' level of utilization of social services was generally low, possibly due to high case loads and staff overload;

- mothers who used social services more had more life problems that might not have been susceptible to short-term change; 
- the present study did not take into account the content of parents' social services contacts or the nature of the relationship between the family service worker and the parent, dimensions that may be related to outcomes.

The quality of this relationship may be an important mediator of the benefits of social services contacts and should, therefore, be considered in future research on the impact of Head Start social services.

Although a major goal of the social services component is to aid in identifying opportunities for increasing self-sufficiency, our data did not reveal an association between utilization of social services and parents' self-sufficiency activities during the year following Head Start. (Overall involvement and volunteering, however, did show relationships with aspects of self-sufficiency.) Again, the families who utilized social services more may have had more life problems requiring a greater time period or more intervention for improvement to be documented. Future research could examine the impact of social services on promoting behaviors that are steps on the ladder toward self-sufficiency (such as following through on opportunities, keeping appointments for interviews, being on time when volunteering in the classroom) and that might be more susceptible to change (Herr et al., 1995; Parker et al., 1995). An evaluation of the full potential of the social services component would require an examination of the issues addressed in social services contacts, the quality of parent-staff relationships, and the ratio of social service staff to parent case load.

\section{Summary}

The present study provides evidence of some of the ways in which the parent involvement component of Head Start has achieved its goals. Positive effects of involvement were found with respect to the parent-child relationship, the home learning environment, parents' involvement in their children's kindergarten experience, and parental self-sufficiency. Positive effects of involvement on parental well-being were not found in this study. Aspects of the Head Start child's and sibling's social competence were found to be related to parent involvement. Results showing a differential impact of different types of involvement on outcomes provide new avenues for thinking about the relationship between specific parent involvement activities and specific outcomes.

\section{FULFILLING THE MISSION OF HEAD START AS A TWO-GENERATION PROGRAM}

\section{How Can the Effectiveness of Head Start Parent Involvement be Enhanced?}

Three related challenges for Head Start can be better addressed with information gained from the present study: 
- How to customize services to meet the individual needs of families.

- How to make it easier for parents to participate in and benefit from involvement in Head Start.

- How to reconcile the new welfare-to-work demands on parents' time with Head Start's present requirements for parents' participation.

Embedded in these challenges are five important questions regarding the conceptualization and measurement of parent involvement that suggest avenues by which future research and policy can help shape an even more effective parent involvement program. These questions are especially timely in light of the current challenge of defining an effective Head Start parent involvement component for the 21st century within the context of current economic and political realities such as welfare reform (Parker et al., 1995).

\section{How should parent involvement be measured?}

The present study measured parent involvement as the number of hours of participation. This metric, while revealing some positive associations with outcomes, may be underestimating the full impact of Head Start. An important next step would entail examining the quality of the parent involvement process, including staff-parent relationships, the parents' experience of their involvement, and the skills and preferences that parents bring to their experience. ${ }^{8}$

\section{Should measures of involvement include at-home behaviors?}

Activities not currently taken into account in measuring parent involvement in Head Start are those initiated by Head Start staff to be carried out at home (such as phoning other parents to schedule a meeting, or engaging in a teacher-initiated learning activity with the Head Start child at home). This type of participation could not be assessed in the present study because records of parents' participation in these types of activities are not routinely kept by these (and most) Head Start agencies. An important next step would be to measure and assess the impact of at-home parent involvement on parents and children.

\section{Is there a minimum threshold of involvement?}

In the present study, overall involvement at Agency One was substantially lower than in the previous study at the same agency (Parker et al., 1987). The high-involved mothers in this study were at a comparable level of involvement to those mothers who were low involved in the earlier study. In the earlier study, the medium- and high-involved mothers benefitted but not the low-involved mothers. As levels of involvement appear to vary across time and context, an important next step in parent involvement research 
could entail identifying the minimum amount of participation in Head Start -- in conjunction with participation in other activities -- that leads to positive outcomes for parents.

Future research should also continue to systematically identify the barriers to fuller involvement in order to increase the likelihood that parents can be involved in Head Start. One possible cause of lower involvement is the present harsher economic climate in which a greater percentage of mothers (33\% in the current sample versus $18 \%$ in the 1987 sample) are engaged in self-sufficiency-promoting activities (working, attending school, job training) outside the Head Start center. Our data showed that engagement in self-sufficiency-promoting activities is associated with a decrease in Head Start participation. Thus, these activities compete with traditional Head Start activities for parents' limited time and resources. Lower participation may also be due to insufficient funds to include all parents in workshops and unrealistically high parent involvement/social services staff case loads.

How does involvement in Head Start combine with parents' self-sufficiency-promoting activities to affect outcomes?

Although a causal relationship cannot be established from our data, the present study found an association between working, going to school, or being in job training and reduced participation in Head Start. Our post hoc analyses suggested that the processes leading to positive outcomes may be different for Head Start parents who are also working, going to school, or in job training. Parents engaged in these activities in addition to their high Head Start involvement may become overloaded and stressed, resulting in negative outcomes for parents and children. This issue addresses the related challenge of how to reconcile participation in Head Start with the new welfare-to-work laws, which place further demands on parents' time, and how to make it easier for busy parents to participate in Head Start.

What is the relationship of Head Start to welfare-to-work programs that promote selfsufficiency?

Head Start parents will be increasing their engagement in self-sufficiency-promoting activities outside Head Start through mandatory participation in the new welfare-to-work programs. Both Head Start and welfare-to-work programs share the goal of helping parents engage in job training or education leading to paid employment. Parker et al., (1995) suggest that helping parents become self-sufficient is a component of the Head Start program that has not attained maximum effectiveness because of insufficient funds and staff training. Herr and Halpern (1991) propose that creating a partnership between Head Start and welfare-to-work programs would provide both with additional resources that would help each fulfill its objective. Since Head Start is perceived by parents as a friendly, supportive environment that provides sound early childhood education and outof-home care, parents might be amenable to participating in self-sufficiency activities on

H/S Exec. Summary -- Page 20 
site. In fact, parents who participated in our Barriers Survey (see Part I) expressed a desire for these activities at their Head Start center. By receiving such services on-site, parents' travel time and expenses may be reduced, leaving them with more time to learn the skills needed to attain self-sufficiency. The partnership might also be cost effective by reducing some duplication of services. An additional benefit could be the inclusion of these activities as in-kind services for the Head Start program.

In summary, helping parents receive maximum benefit from Head Start and attain selfsufficiency in part depends on understanding how all of the activities parents are involved in, both in and out of Head Start, together affect parents and families. Research can shed light on this process by identifying and providing a deeper understanding of all of the major forces impinging on the family.

\section{Recommendations for Future Research}

The following recommendations for future research would add substantively to the ability to document and understand the ways in which parent involvement in Head Start has a positive impact on the lives of Head Start parents and their families. Such information is vital as Head Start moves into the 21st century:

- Identify groups of parents who differ on background characteristics or on their combination of parent involvement experiences (e.g., high in workshops/policy meetings but low in volunteering) who may have different outcomes.

- Examine possible interaction effects between involvement and variables other than self-sufficiency. For example, involvement may have a greater positive impact on parental aggravation for less strict parents than for stricter parents.

- Examine the content and quality of parents' social services contacts and relate these to improved outcomes.

- Identify additional predictors of involvement related to the characteristics of Head Start programs, neighborhoods and communities.

- Continue to develop a process model in which specific involvement activities are linked to specific types of outcomes.

- Examine the effects of involvement on additional outcomes that would best capture the benefits of the Head Start experience (e.g., child health outcomes).

- Examine outcome data from a cultural perspective to better understand culturally based beliefs, perceptions, and responses with regard to the major outcome constructs. 


\section{Programmatic and Policy Recommendations}

The following is a summary of the policy and programmatic recommendations:

- Reexamine the needs and skills of parents and how Head Start can address these through the types of involvement activities offered.

- Tailor programs to meet parents' specific goals, guided by a model of which aspects of parent involvement have which outcomes.

- Redefine Head Start parent involvement to include activities initiated by the Head Start center to be carried out by the parent at home (e.g., reading to the Head Start child, volunteering on a Head Start fund-raising activity).

- Reduce the case loads of social services staff to maximize their ability to build strong partnerships with parents and address parents' individual needs so that they can attain their goals.

- Add services to decrease barriers to fuller participation in Head Start (e.g., wraparound child care, full-day program for Head Start child).

- Examine the feasibility of instituting state-level coordination between Head Start agencies and welfare-to-work programs so that Head Start agencies can offer onsite job training and educational activities leading to paid employment as part of their parent involvement component. 


\section{PART III: THE INTERVENTION: STRATEGIES TO ENHANCE STAFF'S WORK WITH PARENTS: A PROCESS OF SELF-AWARENESS, LEARNING, AND EMPOWERMENT}

Faith Lamb Parker, Chaya S. Piotrkowski, Susan Young, Beryl Clark, Lenore Peay, Cynthia Flynn, \& Amy J. L. Baker.

ABSTRACT
A social services/parent involvement staff workshop and ongoing peer group were
developed to train staff to work with hard-to-engage Head Start mothers. Qualitative
evaluations suggested that staff gained insight into their own and mothers' attitudes,
feelings, and values. Staff also reported that they developed increased empathy for
mothers and increased ability to interact productively with them to reduce barriers to
participation and to customize activities based on mothers' needs, strengths, and
resources.

\section{INTRODUCTION}

There is a longstanding tradition in Head Start of hiring Head Start parents for staff positions. Thus, former Head Start parents function as social services/parent involvement staff. These staff members reside in the community served by the Head Start program, have paraprofessional status, and, consequently, receive low wages. Therefore, Head Start staff frequently experience life circumstances similar to those of the Head Start parents they serve and are often vulnerable to many of the same stressors. In order to work more effectively with parents, staff need support in developing and maximizing their personal and professional skills, including their emotional strength, ingenuity, and creativity. If staff lack an in-depth understanding of their own circumstances, including their conscious and unconscious values and attitudes, they will have fewer personal and professional resources with which to work effectively with parents.

Unfortunately, there are a limited number of training opportunities for social services/parent involvement staff that focus on staff support and development. ${ }^{9}$ Therefore, the Parent Involvement Project's HSRG developed and implemented a stafffocused intervention to help meet this need.

\section{DEVELOPING THE INTERVENTION}

In order to provide a theoretical and empirical foundation for addressing staff issues, the HSRG took several steps: 1) reviewing research on issues relevant to barriers to parents' 
participation in their children's school experience (the effects of depressive symptoms and demoralization on parents and children, relationship-building with a focus on partnerships when working with parents, and cultural traditions and values that may affect parent involvement in Head Start and public school settings); 2) surveying Head Start mothers (see Part I) to assess potential barriers to their participation in Head Start; and 3) conducting focus groups with social services staff about working with parents, considering their personal feelings, professional needs, and possible solutions for enhancing parents' participation in the program.

At the end of this process, the HSRG reached consensus that the major thrust of the intervention would be on staff attitudes toward and feelings about their work with parents and on social services/parent involvement staff's ongoing relationship with parents. To that end, two staff development and training strategies were developed: 1) a two-day workshop for all Head Start staff; and 2) an ongoing peer group for social services/parent involvement staff. The strategies were developed to help staff better understand how to work more effectively with parents, especially those whom they perceived to be particularly hard to engage ${ }^{10}$ in the Head Start experience. They were designed to provide staff with: 1) a deeper understanding of their and parents' strengths, needs, attitudes, values, and goals; 2 ) key psychosocial concepts related to child and family development; 3 ) more appropriate opportunities for enhancing parents' participation; 4) peer support; 5) problem-solving strategies; and 6) oral and written skill-building opportunities. The overarching aim was to empower both staff and parents.

The two-day workshop consisted of a series of nine interactive exercises that explored staff perceptions of and attitudes toward Head Start parents, especially those whom they felt were hard to engage, uncovered hidden prejudices; addressed staffs' and parents' stressors and supports and how they related to parent involvement; and linked parent involvement to empowerment. The workshop was conducted separately at each agency. All staff $(\mathrm{N}=82)$ participated, including consultants and maintenance personnel.

The peer group for social services/parent involvement staff was developed to provide staff with an opportunity to meet regularly with peers to further develop their understanding and skills in their work with a subset of hard-to-engage parents. The Peer Group included a facilitator who was knowledgeable in family issues, child development, and group process, and who also had experience in Head Start.

The primary purposes of the peer group were to: 1) provide staff with a supportive professional and emotional environment; 2 ) help them build trusting partnerships with parents to address their needs and goals and enhance their participation; 3 ) address barriers to service delivery and parent involvement; and 4) focus on skill building and professional empowerment. The peer group functioned as a means for staff to work with a case load of hard-to-engage parents and to develop individual and group strategies with parents to enhance their participation in Head Start. Throughout the

H/S Exec. Summary -- Page 24 
sessions, the concepts and lessons learned from the workshop were integrated in to the discussions and plans developed by staff.

\section{SELECTED RESULTS AND DISCUSSION}

\section{The Two-Day Workshop}

Staff responses during the workshop exercises were tallied and qualitatively assessed. It was found that as they moved through the exercises, there was a developmental progression in their understanding of themselves, the Head Start parents, and their role in working with parents. This progression was noted in their understanding of: 1) the complexities of parent involvement; 2) key psychosocial concepts such as the "life load;" 3 ) how to apply these concepts to their work with Head Start parents; and 4) the link between parent involvement and empowerment.

For example, in an early exercise staff revealed a negative attitude toward parents who were less involved, perceiving them to be apathetic about their children's growth and development. In later exercises, however, staff indicated a more complex understanding of the factors in parents' lives that competed or interfered with their involvement. Discussions included an acknowledgement that Head Start itself may function as an additional burden for parents, especially if they are pressured to become involved without staff taking into account their needs and goals, such as a desire for job training or further education. This particular exercise focused on the importance of working with parents to determine the most appropriate resources and activities for them.

The developmental progression of staffs' understanding of themselves, Head Start parents, and their role in working with parents was also reflected in their evaluation of the effectiveness of the workshop. First, staff explessed almost unanimous agreement that the workshop had been beneficial to them and that they felt energized by the experience. Specifically, they appreciated the concreteness of the experiential exercises, which enabled them to apply what they learned to their ongoing work with parents. Second, they felt that after participating in the workshop they had a common language and understanding that could be used to discuss various issues pertaining to their work with parents. Third, they mentioned that they had a better understanding of their role vis-à-vis parents and would be better able to work cooperatively with other Head Start staff. Lastly, social services/parent involvement staff expressed eagerness to grapple with issues faced by parents that they had previously avoided, specifically some of the issues and barriers of the hard-to-engage parents. They felt more equipped to do their job. Nonetheless, supervisory staff noted that some staff were still skeptical about how they were going to apply what they learned to their targeted hard-to-engage parents. At the same time, the supervisors noted that staff's general level of willingness seemed improved. 


\section{The Peer Group}

An analysis of the forms filled out by peer group staff on their targeted families as well as the notes taken during each peer group provided both process and outcome information about the effectiveness of the peer group for staff and parents. In these evaluations, several organizational and community barriers to service delivery were revealed. Organizational barriers included insufficient time to complete required paperwork, need for more effective training, too high a caseload, staff turnover, low salaries, and poor working conditions (i.e., violent neighborhoods). Community barriers included a lack of effective community resources. Staff felt that community resources had diminished and that waiting lists for those that remained were so long that referrals became meaningless. Furthermore, when referrals were possible, the bureaucratic red tape of many community agencies, as well as language and cultural barriers, a lack of trust on the parents' part, and/or the geographic distance of the service from the parents' home, made it difficult for parents to stick with the process long enough to receive the appropriate services. Staff felt that these barriers impaired their sense of efficacy in successfully doing their job.

Some of the hard-to-engage mothers who were selected as the focus of staff's work in the peer group shared similar life events and experiences that appeared to be interfering with their involvement, such as working, going to school, having a toddler in the home, low self-esteem, or problematic family relationships. In these instances, staff brainstormed with mothers outside of the peer group and worked with other staff in the peer group to develop approaches that could be implemented with small groups of mothers to address their needs and goals for enhanced involvement. While the approaches were originally developed specifically for these mothers, many of them are appropriate for all Head Start families. ${ }^{11}$

In addition to group approaches, staff also worked closely with individual mothers to address their needs and goals by building on existing strengths and resources. In developing trusting relationships with parents, staff put into practice the concepts learned in the workshop, in particular the concepts of life events and experiences, barriers, relationship building, and empowerment. As they experienced firsthand the issues discussed in the workshop, they became more empathic to and less judgmental of the families, and therefore more effective in their work with them. In fact, staff felt strongly that the peer group substantially improved their effectiveness in working with the families targeted as hard to engage. For example, by the end of the Head Start year, of the 48 mothers targeted for case management, 42 were considered by staff to be "more engaged" in the parent involvement process. Those who were not perceived as improving were facing extreme life situations that the peer group felt were beyond Head Start's scope.

Through the peer group process, staff acquired a deeper understanding of Head Start parents and learned to successfully and effectively develop individualized and group-level 
plans to engage mothers. Through intensive discussions generated by their experiences in the workshop, staff were able to continue their exploration of their own beliefs, attitudes, and values about Head Start parents and developed more empathetic, respectful approaches toward building parent-staff partnerships. They gained confidence in themselves and their ability to foster change in parents' lives. Moreover, they learned how to tailor their approaches to the specific life situation of each parent. They were particularly surprised and touched that seemingly inconsequential gestures turned out to be the delicate and creative overtures that were exactly what hard-to-engage mothers needed in order to establish confidence and trust in the Head Start staff and begin the steps toward involvement and empowerment. In their ongoing work in the peer group, the staff also learned how important their work is for parents.

\section{Summary}

The workshops and peer group intervention enhanced staff both personally and professionally and helped hard-to-engage mothers move into the Head Start parent involvement process. In doing so, it served to accomplish the overall goals that were set by the Head Start Research Group. Specifically, the outcomes for staff that were met included: 1) an increase in self-awareness, and in understanding of and empathy for Head Start parents; 2) the ability to approach barriers to parental participation in positive ways, taking into account the life experiences, strengths, and needs of parents; 3 ) closer peer relationships that served supportive and empowering functions; and 4) an increase in written and oral communication skills. Staff perceived benefits to the targeted hard-to-engage mothers as: 1) an increase in their trust and confidence that Head Start was an important resource and support for them; 2) closer relationships with social services/parent involvement staff that fostered their personal and/or professional development; and 3) an enhancement of their parental participation in Head Start. 


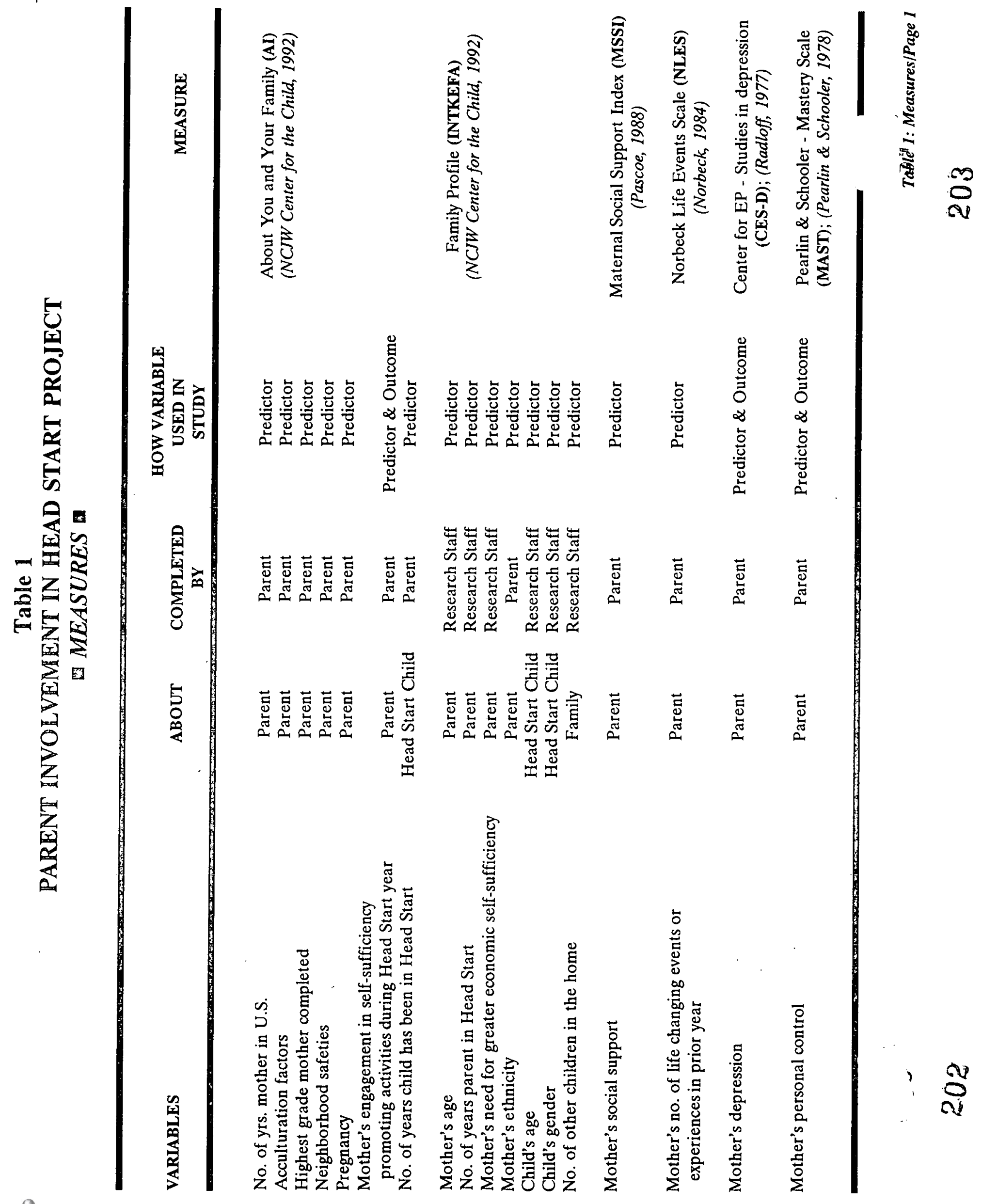




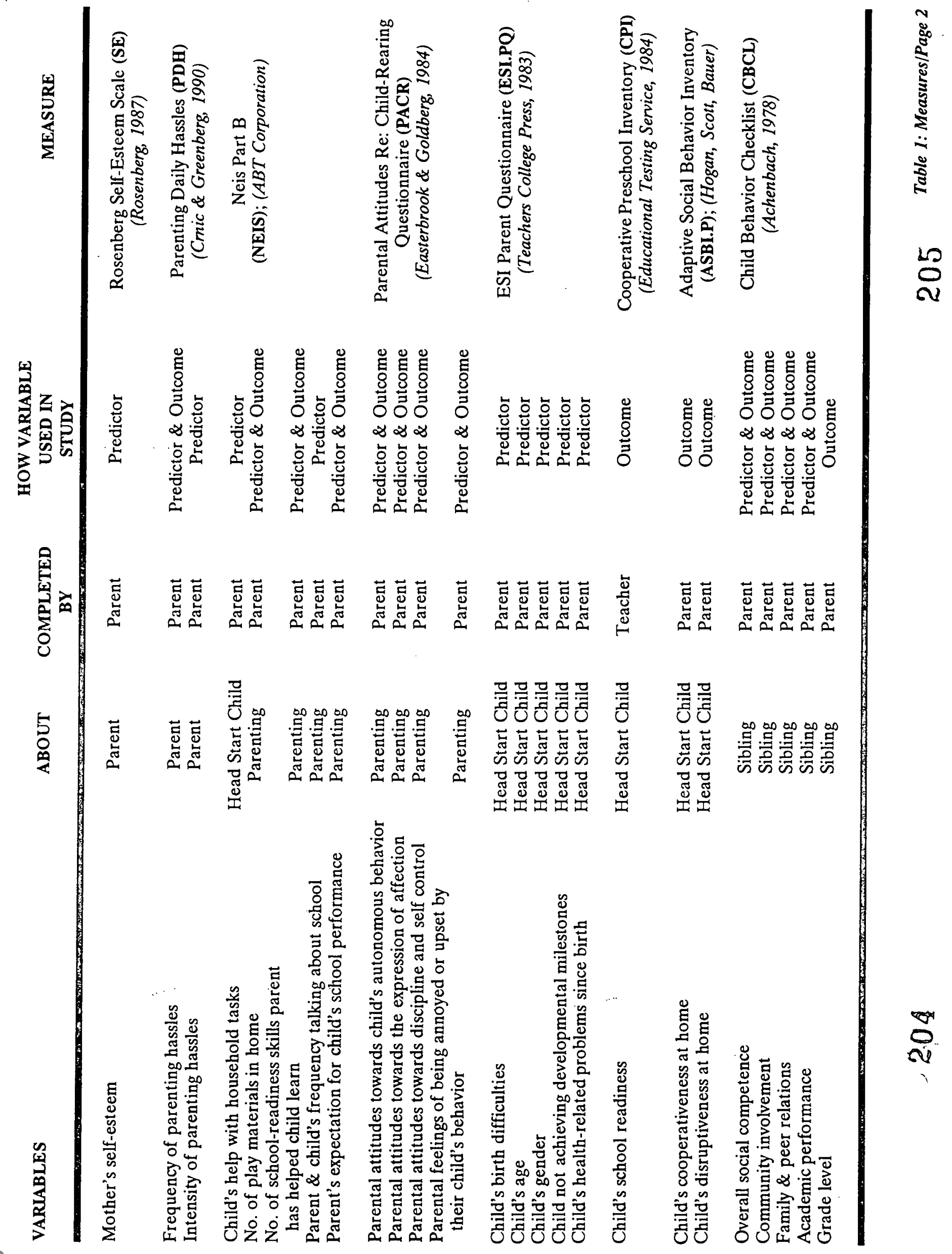




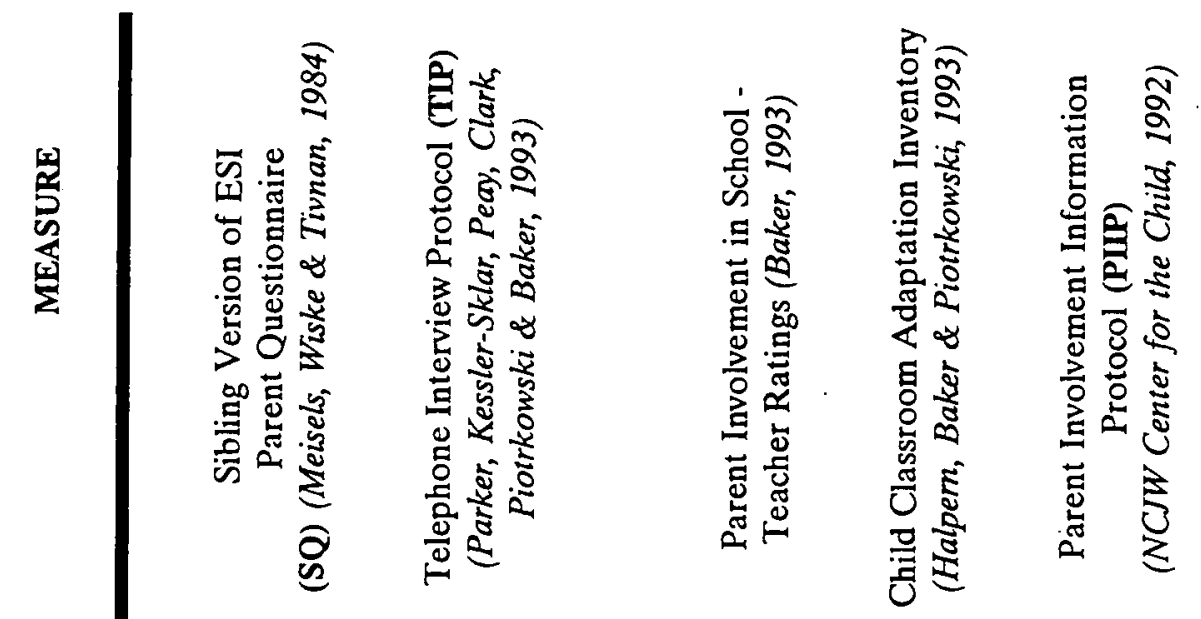

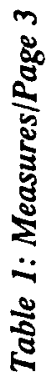

$\sum_{0}^{2}$

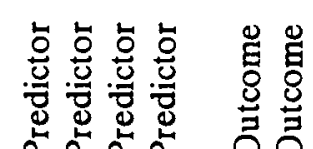

兽 范

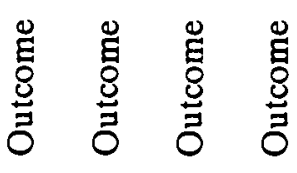

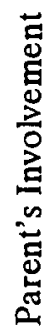

온

节节茅

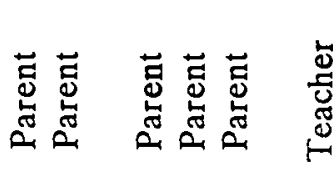

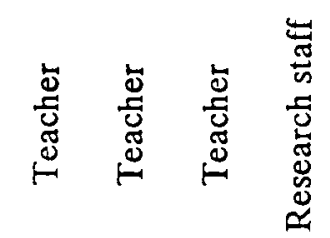

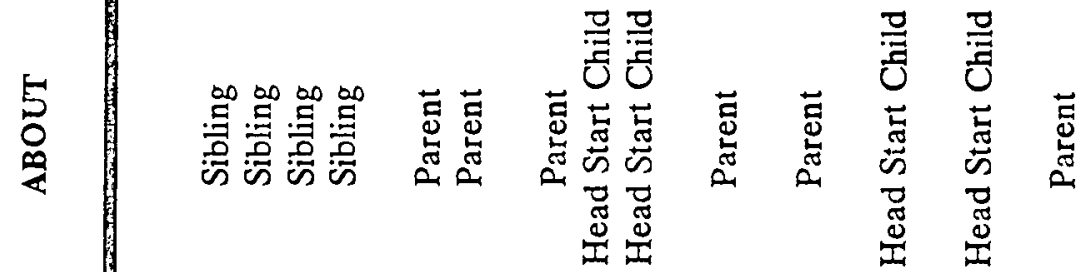

莺莺

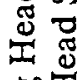

60.工

๕

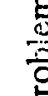

ต

형

完

은

$\circ$ 造

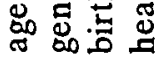

$\overbrace{\infty}^{\infty}$

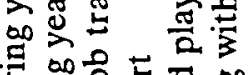

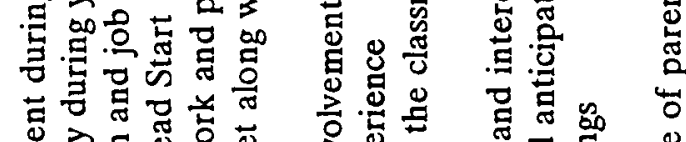

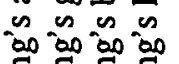

总侥离范

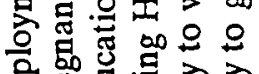

党

留

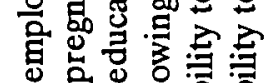

is

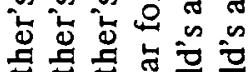

공

ठ 壱

幽要

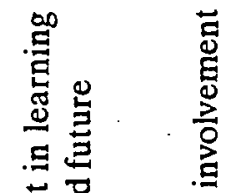

$\underbrace{\infty}_{0}$

$\infty$ 


\section{NOTES}

1. The information for the community profile comes from the following sources: 1) NYC Department of Health, 1988; 1990 US Census; NYC Housing and Vacancy Survey; 1992 NYPD Annual Statistical Report; NYPD Annual Statistical Reports, 1989-1990; cited in Garfield \& Abramson, 1994. 2) Ft. George Community Enrichment Center, inc., Head Start Community Needs Assessment, prepared by Floyd D. Page, 1/31/94.

2. Older siblings of Head Start children between the ages of 5 and 12 were chosen for this study because it was thought that this group might be affected, as a result of parents' participation, by potential changes in their parents' child-rearing attitudes and feelings of personal well-being as well as potential changes in the family's home learning environment. The 12-year-old cutoff age was selected because of the probability of peer-dominated effects in the teenage years.

3. For post hoc analyses, a categorical overall involvement variable was also created to test for nonlinear relationships between involvement and the outcomes. The variable was constructed by dividing the full pretest sample of parents at each agency into three groups -- lowest third, middle third, and highest third -- based on total number of hours spent in parent activities and experiences.

4. Utilization of social services was conceptualized to be qualitatively different from the other three involvement variables. Because parents who utilized social services more tended to have more problems than the average Head Start parent, we hypothesized that the pattern of relationships between this type of involvement and outcomes might be different than that of other types of involvement and outcomes. Data analyses supported these notions. Utilization of social services was not correlated with the other three types of involvement, nor related to the same outcomes.

5. Preliminary analyses of our data indicated that attendance at workshops and participation in policy-making activities needed to be combined because the variability within each of these categories was not sufficient to warrant separate analysis. An additional reason for combining them was that they are theoretically linked as "group-oriented" parent experiences, while volunteering represents an "individual-oriented" parent experience (Herr \& Halpern, 1991).

6. In the 1987 study, volunteering, attendance at workshops and policy meetings, and social services contacts all were correlated, and were included in one measure of involvement. In the present study, social service contacts are not correlated with the other types of involvement and are not included in the measure of overall involvement.

7. For example, volunteering included classroom, office, and kitchen activities as well as assistance on trips -- activities that probably offer parents different skill benefits and experiences.

8. We are currently conducting analyses that may shed light on one of the more complex dimensions of parent involvement by examining the skill benefit derived from different types of participation and the level of initiative required to participate in different parent involvement activities.

9. See ACYF Final Report, Part III, Appendix C, for a summary of current professional development and training opportunities for staff in parent involvement.

H/S Exec. Summary -- Page 28 
10. The term hard to engage was chosen by members of the HSRG to describe those parents who seemed to be participating the least in parent activities and experiences offered by the Head Start program. The HSRG preferred that term over low participating which to them had a more negative connotation. In addition, they liked the term hard to engage because to them it implied a wider range of possible reasons (both positive and negative) behind parents' lack of participation. For example, a parent might be hard to engage because she was in school full-time or in a job-training program, or she might be hard to engage because she felt demoralized and alienated from others or was abusing alcohol. The reasons could also be connected to cultural issues of parents who were new to this country. Therefore, the term hard to engage was chosen for parents who did not seem to be engaged in the Head Start experience for themselves (although they might be bringing their children every day) and did not seem to be taking advantage of the parent-related opportunities that were being offered by the Head Start program.

11. See Part III of the final report to ACYF of the Head Start Parent Involvement Project (Parker, et al., 1996). 


\section{REFERENCES}

Cohen, J. (1977). Statistical power analysis for the behavioral sciences (Rev. ed.). New York: Academic Press.

Collins, R. C. (1993). Head Start: Steps toward a two-generation program strategy. Young Children, 48(2), 25-33, 72-73.

Cook, T. D., \& Campbell, D. T. (1979). Quasi-experimentation. Chicago: Rand McNally.

Duncan, G. J., Brooks-Gunn, J., \& Klebanov, P. K. (1994). Economic deprivation and early childhood development. Child Development, 65, 296-318.

Garfield. R. M. \& Abramson, D. M. (Eds.). (1994). Washington Heights/Inwood: The health of a community. New York: The Health of the Public Program at Columbia University.

Herr, T., \& Halpern, R. (1991). Changing what counts: Re-thinking the journey out of welfare. Evanston, IL: Center for Urban Affairs and Policy Research, Northwestern University.

Herr, T., Halpern, R., \& Majeske, R. (1995). Bridging the worlds of Head Start and welfare-to-work: Building a two-generation self-sufficiency program from the ground up. In I. Sigel (Series Ed.) \& S. Smith (Vol. Ed.), Advances in Applied Developmental Psychology: Two-generation programs for families in poverty. (pp. 161-197). Norwood, NJ: Ablex.

McKey, R. H., Condelli, L., Ganson, H., Barrett, B. J., McConkey, C., \& Plantz, M. C. (1985). The impact of Head Start on children, families, and communities. Final Report of the Head Start Evaluation, Synthesis and Utilization project. (No. 105-81-C-026). Washington, DC: CRS.

National Research Council, National Academy of Sciences. (1996). Beyond the Blueprint: Directions for Research on Head Start's Families. Washington, D.C.: National Academy of Sciences.

National Head Start Association. (May, 1990). Head Start: The nation's pride, a nation's . challenge, recommendations for Head Start in the 1990s. The Report of the Silver Ribbon Panel. Alexandria, VA: Author.

Oyemade, U., Washington, V., \& Gullo D. (1989). The relationship between Head Start parent involvement and economic self-sufficiency of Head Start families. Joumal of Negro Education, 58(1), 5-15. 
Parker, F. L., Piotrkowski, C. S., Clark, B., Peay, L., Yoshikawa, H. (1991). Parent life events/experiences as potential barriers to Head Start parent involvement survey. New York: National Council of Jewish Women Center for the Child.

Parker, F. L., Piotrkowski, C. S., Horn, W. F., \& Greene, S. (1995). The challenge for Head Start: Realizing its vision as a two-generation program. In I. Sigel (Series Ed.) \& S. Smith (Vol. Ed.), Advances in Applied Developmental Psychology. Two generation programs for families in poverty. (pp. 135-159). Norwood, NJ: Ablex.

Parker, F. L., Piotrkowski, C. S., \& Peay, L. (1987). Head Start as a social support for mothers: The psychological benefits of involvement. American Journal of Orthopsychiatry, 57(2), 220-233.

Replogle, E. M. (1995). Head Start as a family support: Renewing a community ethic. Cambridge, MA: Harvard Family Research Project.

Slaughter, D. T., Lindsey, R. W., Nakagawa, K., \& Kuehne, V. S. (1989). Who gets involved? Head Start mothers as persons. The Journal of Negro Education, 58(1), 1629.

U.S. Department of Health and Human Services (1993). Creating a 21st Century Head Start: Final Report of the Advisory Committee on Head Start Quality and Expansion. Washington, D.C.: Author.

U.S. Department of Health and Human Services, Administration on Children, Youth and Families. (1996). An annotated bibliography of Head Start research: 1985-1996. Washington, DC. U.S. Government Printing Office.

U.S. Department of Health and Human Services. (1975; Reissued, 1984; Reprinted 1992; Revised, 1996). Head Start program performance standards. (DHHS Publication No. OHDS 84-31131). Washington, DC: U.S. Government Printing Office.

Valentine, J., \& Stark, E. (1979). The social context of parent involvement in Head Start. In E. Zigler and J. Valentine (Eds.), Project Head Start: A legacy of the war on poverty (pp. 291-313). New York: Free Press.

Zigler, E. (1978). The effect of Head Start: Another look. Educational Psychology, 13, $71-77$.

Zigler, E., \& Muenchow, S. (1992). Head Start: The inside story of America's most successful educational experiment. New York: Basic Books.

Zigler, E., \& Styfco, S. J. (1993). Head Start and Beyond. New Haven, CT: Yale University Press. 
Zigler, E., \& Trickett, P. (1978). IQ, social competence, and evaluation of early childhood education programs. American Psychologist, 33, 789-798.

Zigler, E., \& Valentine, J. (Eds.). (1979). Project Head Start: A legacy of the war on poverty. New York: Free Press. 


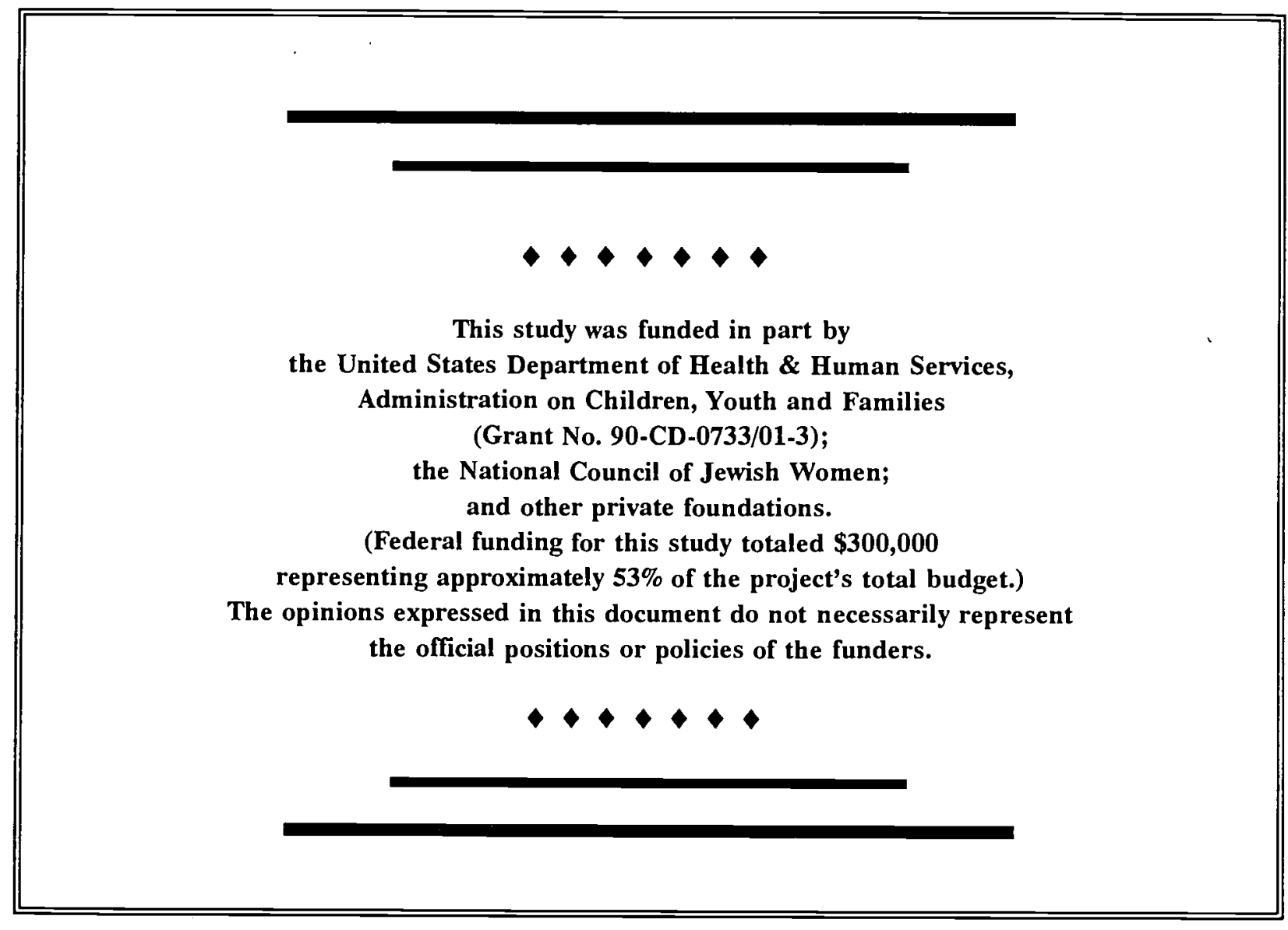



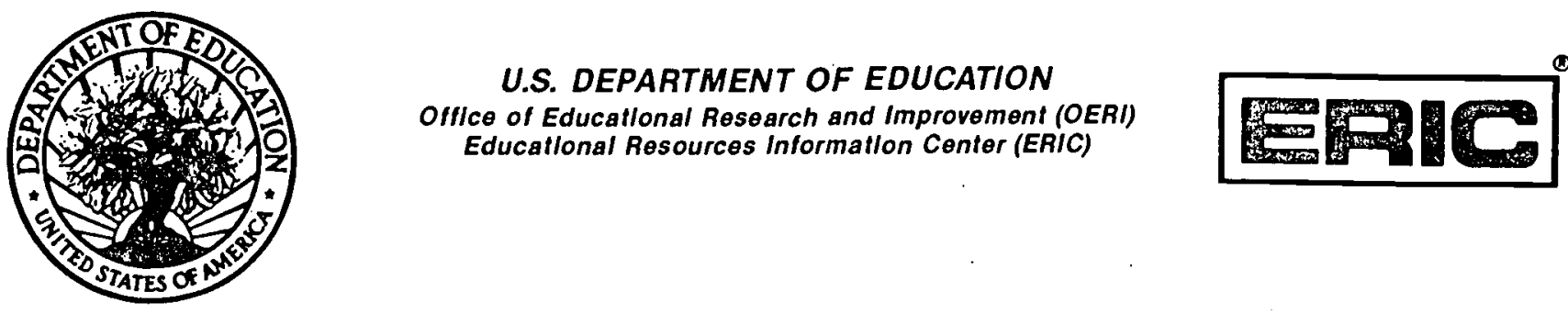

\section{NOTICE}

\section{REPRODUCTION BASIS}

This document is covered by a signed "Reproduction Release (Blanket)" form (on file within the ERIC system), encompassing all or classes of documents from its source organization and, therefore, does not require a "Specific Document" Release form.

This document is Federally-funded, or carries its own permission to reproduce, or is otherwise in the public domain and, therefore, may be reproduced by ERIC without a signed Reproduction Release form (either "Specific Document" or "Blanket"). 